\title{
ELECTROMAGNETIC INTERACTIONS OF MOLECULES WITH METAL SURFACES
}

\author{
G.W. FORD \\ Physics Department, The University of Michigan, Ann Arbor, MI 48109, U.S.A. \\ and
}

W.H. WEBER

Physics Department, Research Staff, Ford Motor Company, Dearborn, MI 48121, U.S.A.

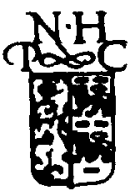

NORTH-HOLLAND PHYSICS PUBLISHING-AMSTERDAM 


\title{
ELECTROMAGNETIC INTERACTIONS OF MOLECULES WITH METAL SURFACES
}

\author{
G.W. FORD \\ Physics Department, The University of Michigan, Ann Arbor, MI 48109, U.S.A. \\ and \\ W.H. WEBER \\ Physics Department, Research Staff, Ford Motor Company, Dearborn, MI 48121. U.S.A.
}

Received April 1984

Contents:

1. Introduction 197

2. Reflection of electromagnetic waves at an interface 198

2.1. Reflection by a nonlocal medium - the SCIB model 201

2.2. The quasistatic approximation 205

2.3. The quantum infinite barrier model 206

2.4. Reflection in the long-wavelength limit 215

3. Molecular fluorescence near a metal 223

3.1. Point dipole above a semi-infinite metal 224

3.2. Power dissipated spectrum 226

3.3. Fluorescence near a single interface 230

3.4. Dielectric layer above a metal 235

3.5. Tunnel junction geometry 237

3.6. Fluorescence near a small sphere $\quad 240$

4. Shift and broadening of molecular vibration modes 243
4.1. Response of a spherical molecule above a nonlocal metal

4.2. Multipole polarizabilities 249

4.3. Shift and broadening of the vibrational mode 254

4.4. Other contributions to the shift 259

4.5. Coverage dependence 261

5. Raman scattering at metal surfaces 265

5.1. Image enhancement 265

5.2. SERS from metal gratings $\quad 271$

5.3. Random roughness 275

5.4. Resonant microstructures, particle arrays and island films $\quad 279$

Appendix A $\quad 284$

References $\quad 285$

Single orders for this issue

PHYSICS REPORTS (Review Section of Physics Letters) 113, No. 4 (1984) 195-287.

Copies of this issue may be obtained at the price given below. All orders should be sent directly to the Publisher. Orders must be accompanied by check.

Single issue price Dfl, 57.00 , postage included. 


\section{Introduction}

The purpose of this article is to describe the electromagnetic interactions of molecules with surfaces, and in particular with metal surfaces. Interest in this subject has been stimulated in recent years by experiments on surface-enhanced Raman scattering, on fluorescence of molecules near metals, and on infrared and inelastic electron spectroscopy of adsorbed molecules. The phenomena observed in the various experiments have much in common from the point of view of electromagnetic theory, and we show how a few general theoretical techniques can be applied to all of them. We do not, however, give a comprehensive review of the experiments. There are a number of such reviews, and we refer to them in the appropriate chapters.

The methods we describe are essentially those of classical electromagnetic theory, the idea being that the molecule and the surface are separated so that their only interaction is via the electromagnetic fields. This means that we leave out important quantum mechanical effects, such as the hybridization of the molecular orbitals with the electronic wavefunctions in the metal. Such effects could be addressed using quantum mechanical approaches, such as the Hartree-Fock or density functional methods. These methods, however, are in practice limited to ground-state properties and can give little insight into the dynamical properties we consider. These methods are also valid only very near the surface. Far away from the surface they fail to describe effects arising from electromagnetic interactions, an example being the Van der Waals force attracting a molecule to a surface. In a certain sense the electromagnetic and quantum mechanical methods are complementary, the former describing the distant interactions, the latter the very short-range interactions. Our intention is to press the electromagnetic calculations as close to the surface as is feasible. When the quantum mechanical effects neglected in our discussion are small, as in the case of molecular fluorescence, quantitative comparisons between theory and experiment can be made. When they are not small, or when they are difficult to estimate, as in the case of surface-enhanced Raman scattering, the comparisons will be less quantitative. In these cases the value of our treatment of a particular electromagnetic effect will be in the insight provided by a careful calculation of that one aspect of the problem.

Some of the processes we do consider, such as molecular fluorescence, are basically quantum mechanical in nature. However, they can be described within the framework of the so-called semiclassical theory of radiation. In this theory the electromagnetic interactions are described by classical Maxwell equations, while the classical source strength is expressed in terms of the quantum mechanical transition matrix elements [1]. To invoke a fully quantum mechanical formalism to describe such processes would, in our view, obscure the physical insight gained from the results.

This article is organized as follows. In chapter 2 we discuss the reflection of electromagnetic waves at a plane interface, showing how to extend the classical Fresnel formulas to the case of a nonlocal medium. This is the basic theoretical ingredient that we use in the discussion of the various phenomena in succeeding chapters. Two commonly used models for calculating the surface response of a metal are described in detail: (1) the semiclassical infinite barrier model, in which the electron density is uniform up to the barrier, where it abruptly goes to zero, and (2) the quantum infinite barrier model, in which the electron density undergoes Friedel-type oscillations near the surface and goes smoothly to zero at the barrier. Also discussed are models that allow for a more general form of the electron density profile at the surface, but which to date have only been solved in the long-wavelength limit.

In chapter 3 we discuss molecular fluorescence near a metal. Here the theoretical problem is that of the radiation by an oscillating dipole above a nonlocal metal, and we solve it by superposing the waves constructed in chapter 2 . We use the result to discuss the decay into various modes such as surface 
plasmons and electron-hole excitations. We also extend the results of chapter 2 to the case of layered structures and compare the results with recent experiments. Finally, still in chapter 3 we consider molecular fluorescence near a small metal sphere, introducing the multipole polarizabilities, which are the spherical analogs of the plane-wave reflection coefficients.

In chapter 3 the fluorescing molecule is treated as a radiating dipole whose decay rate is modified by the induced fields (image fields) from the metal but whose frequency and transition moment are not. In the following two chapters, devoted, respectively, to infrared absorption and Raman scattering by adsorbed molecules, we consider problems in which the effect of these induction fields is significant, and the renormalization of the radiating dipole is required. In chapter 4 we show how to include such effects in a consistent calculation of the shift and broadening of a vibrational absorption line in an adsorbed molecule. We take into account finite molecular size and nonlocal metal response, aspects of the calculation already introduced in chapters 2 and 3 . We compare the results with experiments on $\mathrm{CO}$ adsorbed on $\mathrm{Cu}$.

In chapter 5 we first apply the theoretical ingredients introduced in the previous chapters to discuss the enhancement of Raman scattering for a molecule adsorbed on a smooth surface. The result is a physically consistent calculation of what is termed the image enhancement effect: the increase in the dipole moment induced in the molecule due to the image fields from the metal. We then consider the effects of various types of surface roughness on the Raman scattering of adsorbed molecules, first for the case of periodic gratings ruled onto the surface, then for the case of random roughening, and finally for the case of discrete metal particles that have a resonant polarizability. The effects associated with the polarizability resonances of discrete metal particles lead to what is commonly referred to as the "electromagnetic explanation" for surface-enhanced Raman scattering. We critically examine this explanation in comparison with experimental results.

\section{Reflection of electromagnetic waves at an interface}

In this chapter we discuss the reflection and transmission of electromagnetic waves incident upon a surface. As a preliminary step and to fix the notation, we begin with the Maxwell equations, which in Gaussian units take the form $[2,3]$

$$
\operatorname{div} \boldsymbol{B}=0, \quad \operatorname{curl} \boldsymbol{E}+\frac{1}{c} \frac{\partial \boldsymbol{B}}{\partial t}=0, \quad \operatorname{div} \boldsymbol{D}=4 \pi \rho, \quad \operatorname{curl} \boldsymbol{B}-\frac{1}{c} \frac{\partial \boldsymbol{D}}{\partial t}=\frac{4 \pi}{c} \boldsymbol{j}
$$

Here the second equation is Faraday's law of induction, the third is Gauss's law, and the fourth we call Ampère's law, although historically Ampère's law did not have the displacement current term. For fields varying harmonically in time $\left[\boldsymbol{E}(\boldsymbol{r}, t)=\boldsymbol{E}(\boldsymbol{r}) \mathrm{e}^{-\mathrm{i} \omega t}\right.$, and similarly for the other fields $] \dagger$ Faraday's and Ampère's laws become

$$
\operatorname{curl} \boldsymbol{E}-\mathrm{i} \frac{\omega}{c} \boldsymbol{B}=0, \quad \operatorname{curl} \boldsymbol{B}+\mathrm{i} \frac{\omega}{c} \boldsymbol{D}=\frac{4 \pi}{c} \boldsymbol{j} .
$$

The first and third equations in (2.1) follow from these and the continuity equation

† We make extensive use of a notation in which the same symbol stands for a quantity with and without an exponential factor, here $\mathrm{e}^{-i \omega t}$. It should always be clear from the context which quantity is to be understood. 


$$
-\mathrm{i} \omega \rho+\operatorname{div} j=0
$$

For a local medium the dielectric relation between the electric displacement $\boldsymbol{D}$ and the electric field $\boldsymbol{E}$ takes the form

$$
\boldsymbol{D}(\boldsymbol{r})=\varepsilon(\omega) \boldsymbol{E}(\boldsymbol{r})
$$

where $\varepsilon(\omega)$ is the frequency-dependent dielectric constant. Here the term "local" refers to the fact that (2.4) relates $\boldsymbol{D}$ and $\boldsymbol{E}$ at the same point in space. For an infinite homogeneous but nonlocal medium, the dielectric relation is simply expressed as a relation between plane wave amplitudes of given frequency $\omega$ and wavevector $\boldsymbol{k}:[\boldsymbol{E}(\boldsymbol{r}, t)=\boldsymbol{E} \exp [\mathrm{i}(\boldsymbol{k} \cdot \boldsymbol{r}-\omega t)]$, etc. $]$

$$
\boldsymbol{D}=\varepsilon_{\ell}(k, \omega) \hat{\boldsymbol{k}} \cdot \boldsymbol{E} \hat{\boldsymbol{k}}+\varepsilon_{\mathrm{t}}(k, \omega)(\boldsymbol{E}-\hat{\boldsymbol{k}} \cdot \boldsymbol{E} \hat{\boldsymbol{k}}),
$$

where $\varepsilon_{\ell}$ and $\varepsilon_{\mathrm{t}}$ are, respectively, the longitudinal and transverse dielectric constants.

The simplest reflection problem is that in which a plane wave is incident upon the plane boundary between two homogeneous media described by local dielectric constants $\varepsilon_{1}(\omega)$ and $\varepsilon_{2}(\omega)$. In this geometry one distinguishes between P-polarized waves, in which the magnetic field of the wave is parallel to the interface, and S-polarized waves, in which the electric field is parallel to the interface. The reflection and transmission coefficients are then given by the well-known Fresnel formulas. In the P-polarized case these are

$$
r_{12}^{\mathrm{P}}=\left(q_{1} \varepsilon_{2}-q_{2} \varepsilon_{1}\right) /\left(q_{1} \varepsilon_{2}+q_{2} \varepsilon_{1}\right), \quad t_{12}^{\mathrm{P}}=2 q_{1} \varepsilon_{2} /\left(q_{1} \varepsilon_{2}+q_{2} \varepsilon_{1}\right)
$$

and in the S-polarized case they are

$$
r_{12}^{\mathrm{s}}=\left(q_{1}-q_{2}\right) /\left(q_{1}+q_{2}\right), \quad t_{12}^{\mathrm{s}}=2 q_{1} /\left(q_{1}+q_{2}\right) \text {. }
$$

In these formulas the incident wave has been chosen to be in the medium with dielectric constant $\varepsilon_{1}$, and

$$
q_{j}=\left(\varepsilon_{j} \omega^{2} / c^{2}-p^{2}\right)^{1 / 2}, \quad \operatorname{Im} q_{j}>0
$$

where $p$ is the component of the wavevector parallel to the interface.

The derivation of the Fresnel formulas appears in any textbook on electrodynamics [2-5]. However, to fix the notation, a brief derivation might be useful here. We choose a coordinate system in which the $x y$ plane is the boundary between the two media, with the medium above the plane (positive $z$ ) having dielectric constant $\varepsilon_{1}(\omega)$ and that below having dielectric constant $\varepsilon_{2}(\omega)$. We write the coordinate vector $\boldsymbol{r}=\boldsymbol{\rho}+z \hat{\boldsymbol{z}}$, where $\boldsymbol{\rho}=x \hat{\boldsymbol{x}}+y \hat{\boldsymbol{y}}$ is the component parallel to the interface. The fields vary harmonically in time. The incident wave is in the medium above the surface, propagating downward. Then for $\mathrm{P}$-polarized waves the magnetic field in the two media has the form

$$
\boldsymbol{B}(\boldsymbol{r})=B_{\mathrm{P}} \exp (\mathrm{ip} \cdot \boldsymbol{\rho}) \hat{\boldsymbol{z}} \times \hat{\boldsymbol{p}} \begin{cases}\exp \left(-\mathrm{i} q_{1} z\right)+r_{12}^{\mathrm{P}} \exp \left(\mathrm{i} q_{1} z\right), & z>0 \\ t_{12}^{\mathrm{P}} \exp \left(-\mathrm{i} q_{2} z\right), & z<0\end{cases}
$$


where $\boldsymbol{p}$ is the component of the wavevector parallel to the surface and $B_{\mathrm{P}}$ is a complex amplitude. The electric field is then given by Ampère's law,

$$
\boldsymbol{E}(\boldsymbol{r})=\frac{c}{\omega} B_{\mathrm{P}} \exp (\mathrm{i} \boldsymbol{p} \cdot \boldsymbol{\rho}) \begin{cases}-\frac{q_{1} \hat{\boldsymbol{p}}+p \hat{z}}{\varepsilon_{1}} \exp \left(-\mathrm{i} q_{1} z\right)+\boldsymbol{r}_{12}^{\mathrm{P}} \frac{q_{1} \hat{\boldsymbol{p}}-p \hat{z}}{\varepsilon_{1}} \exp \left(\mathrm{i} q_{1} z\right), & z>0 \\ -t_{12}^{\mathrm{P}} \frac{q_{2} \hat{\boldsymbol{p}}+p \hat{z}}{\varepsilon_{2}} \exp \left(-\mathrm{i} q_{2} z\right), & z<0 .\end{cases}
$$

Faraday's law now requires

$$
p^{2}+q_{j}^{2}=\varepsilon_{j} \omega^{2} / c^{2}, \quad \operatorname{Im} q_{j}>0
$$

where the sign of the imaginary part of $q_{1}$ has been chosen so the waves are damped in the direction of propagation. Finally, the requirement that the components of $\boldsymbol{B}$ and $\boldsymbol{E}$ parallel to the interface be continuous gives

$$
1+r_{12}^{\mathrm{P}}=t_{12}^{\mathrm{P}}, \quad\left(q_{1} / \varepsilon_{1}\right)\left(1-r_{12}^{\mathrm{P}}\right)=\left(q_{2} / \varepsilon_{2}\right) t_{12}^{\mathrm{P}},
$$

from which we obtain the formulas (2.6).

In the same way, for S-polarized waves the electric field has the form

$$
\boldsymbol{E}(\boldsymbol{r})=E_{\mathrm{S}} \exp (\mathrm{i} \boldsymbol{p} \cdot \boldsymbol{\rho}) \hat{z} \times \hat{\boldsymbol{p}} \begin{cases}\exp \left(-\mathrm{i} q_{1} z\right)+r_{12}^{\mathrm{s}} \exp \left(\mathrm{i} q_{1} z\right), & z>0 \\ t_{12}^{\mathrm{s}} \exp \left(-\mathrm{i} q_{2} z\right), & z<0\end{cases}
$$

The magnetic field is now given by Faraday's law,

$$
\boldsymbol{B}(\boldsymbol{r})=\frac{c}{\omega} E_{\mathrm{S}} \exp (\mathrm{i} \boldsymbol{p} \cdot \boldsymbol{\rho}) \begin{cases}\left(q_{1} \hat{\boldsymbol{p}}+p \hat{z}\right) \exp \left(-\mathrm{i} q_{1} z\right)+r_{12}^{\mathrm{s}}\left(-q_{1} \hat{\boldsymbol{p}}+p \hat{\boldsymbol{z}}\right) \exp \left(\mathrm{i} q_{1} z\right), & z>0 \\ t_{12}^{\mathrm{s}}\left(q_{2} \hat{\boldsymbol{p}}+p \hat{z}\right) \exp \left(-\mathrm{i} q_{2} z\right), & z<0\end{cases}
$$

Continuity of the components of $\boldsymbol{E}$ and $\boldsymbol{B}$ parallel to the interface now requires

$$
1+r_{12}^{\mathrm{s}}=t_{12}^{\mathrm{s}}, \quad q_{1}\left(1-r_{12}^{\mathrm{s}}\right)=q_{2} t_{12}^{\mathrm{s}}
$$

from which we obtain the formulas (2.7).

The Fresnel formulas are accurate for many applications, but when applied to the case in which the fields vary on an atomic scale, there are serious discrepancies. Our first aim in this chapter will therefore be to formulate the appropriate generalization of the classical Fresnel formulas. This we do in section 2.1, where we introduce the concept of surface impedance and use it to construct the reflection coefficient for the so-called semiclassical infinite barrier (SCIB) model of a metal. In section 2.2 we discuss the quasistatic approximation. In section 2.3 we discuss the quantum infinite barrier (QIB) model. Finally, in section 2.4 we discuss corrections to the Fresnel formulas in the long-wavelength limit, obtaining formulas that can be evaluated for other models with a smooth transition of the electron density across the interface. 


\subsection{Reflection by a nonlocal medium - the SCIB model}

We here consider the reflection of plane electromagnetic waves propagating in a medium with (local) dielectric constant $\varepsilon_{1}(\omega)$ and incident upon a half-space in which the medium has a general nonlocal response. As in the above discussion of the Fresnel formulas, we choose the boundary between the two media to be the $x y$ plane, with the local medium above the plane called medium 1 and the nonlocal medium below the plane called medium 2 . The fields in medium 1 are therefore of the same form as in the Fresnel case. Thus, in the P-polarized case, as in (2.9) and (2.10), we can write

$$
\begin{aligned}
& \boldsymbol{E}(\boldsymbol{r})=-\frac{c}{\varepsilon_{1} \omega} B_{\mathrm{P}} \exp (\mathrm{ip} \cdot \boldsymbol{\rho})\left[\left(p \hat{z}+q_{1} \hat{\boldsymbol{p}}\right) \exp \left(-\mathrm{i} q_{1} z\right)+r_{12}^{\mathrm{P}}\left(p \hat{z}-q_{1} \hat{\boldsymbol{p}}\right) \exp \left(\mathrm{i} q_{1} z\right)\right], \\
& \boldsymbol{B}(\boldsymbol{r})=B_{\mathrm{P}} \exp (\mathrm{i} \boldsymbol{p} \cdot \boldsymbol{\rho}) \hat{\boldsymbol{z}} \times \hat{\boldsymbol{p}}\left\lfloor\exp \left(-\mathrm{i} q_{1} z\right)+\boldsymbol{r}_{12}^{\mathrm{P}} \exp \left(\mathrm{i} q_{1} z\right)\right\rfloor, \quad z>0 .
\end{aligned}
$$

Similarly, in the S-polarized case, as in (2.13) and (2.14), we can write

$$
\begin{aligned}
& \boldsymbol{E}(\boldsymbol{r})=E_{\mathrm{S}} \exp (\mathrm{i} \boldsymbol{p} \cdot \boldsymbol{\rho}) \hat{\boldsymbol{z}} \times \hat{\boldsymbol{p}}\left[\exp \left(-\mathrm{i} q_{1} z\right)+r_{12}^{\mathrm{s}} \exp \left(\mathrm{i} q_{1} z\right)\right], \\
& \boldsymbol{B}(\boldsymbol{r})=\frac{c}{\omega} E_{\mathrm{S}} \exp (\mathrm{i} \boldsymbol{p} \cdot \boldsymbol{\rho})\left[\left(p \hat{z}+q_{1} \hat{\boldsymbol{p}}\right) \exp \left(-\mathrm{i} q_{1} z\right)+r_{12}^{\mathrm{s}}\left(p \hat{z}-q_{1} \hat{p}\right) \exp \left(\mathrm{i} q_{1} z\right)\right], \quad z>0 .
\end{aligned}
$$

In these expressions, as in (2.8),

$$
q_{1}=\left(\varepsilon_{1} \omega^{2} / c^{2}-p^{2}\right)^{1 / 2}, \quad \operatorname{Im} q_{1}>0
$$

When the medium 2 is nonlocal, it is convenient to formulate the discussion in terms of the surface impedances, as defined by Landau and Lifshitz [3] and by Garcia-Moliner and Flores [6],

$$
Z^{\mathrm{P}}(p, \omega)=-\frac{4 \pi}{c}\left\{\frac{\hat{\boldsymbol{p}} \cdot \boldsymbol{E}}{\hat{\boldsymbol{z}} \times \hat{\boldsymbol{p}} \cdot \boldsymbol{B}}\right\}_{\text {inside }}, \quad Z^{\mathrm{S}}(p, \omega)=\frac{4 \pi}{c}\left\{\frac{\hat{\boldsymbol{z}} \times \hat{\boldsymbol{p}} \cdot \boldsymbol{E}}{\hat{\boldsymbol{p}} \cdot \boldsymbol{B}}\right\}_{\text {inside }}
$$

where the fields are evaluated just inside the medium $2 . \dagger$ Since the components of the electric and magnetic fields parallel to the interface are always continuous across the interface, the surface impedances must be equal to the same expressions evaluated just outside the medium 2 , where the fields have the form (2.16-2.17). We obtain from this the following expressions for the reflection coefficients in terms of the surface impedances:

$$
r_{12}^{\mathrm{P}}=\frac{4 \pi q_{1} / \omega \varepsilon_{1}-Z^{\mathrm{P}}}{4 \pi q_{1} / \omega \varepsilon_{1}+Z^{\mathrm{P}}}, \quad r_{12}^{\mathrm{s}}=\frac{Z^{\mathrm{s}}-4 \pi \omega / c^{2} q_{1}}{Z^{\mathrm{S}}+4 \pi \omega / c^{2} q_{1}}
$$

Thus, a knowledge of the surface impedances is equivalent to a knowledge of the reflection coefficients. The advantage gained by introducing the surface impedances is that they can be expressed entirely in terms of the solutions of the field equations within the medium 2 alone.

\footnotetext{
†In this formulation it would appear that the surface of the medium 2 would have to be abrupt, with no material properties, e.g., conduction electrons in a metal, extending outside the surface. However, we can still use these definitions if we choose the interface between "inside" and "outside" to be placed a small distance outside the actual surface.
} 
The calculation of the surface impedances depends upon the description of the medium below the surface. For a metal the simplest approach that includes the features of nonlocality is the semiclassical infinite barrier (SCIB) model, which corresponds to an assumption of specular reflection of the conduction electrons at the metal surface. This model was first discussed by Reuter and Sondheimer [7], in connection with the anomalous skin effect, and later by Kliewer and Fuchs [8]. The idea is that the conduction electrons are treated as a classical ideal gas, but with Fermi-Dirac statistics. Then, from a consideration of the Boltzmann equation for the electrons, one sees that when the reflection is specular, the fields within the metal half-space are identical to the fields in an infinite metal arising from a source corresponding to a current sheet at the surface. A picture illustrating this model is shown in fig. 1. The heavy horizontal line represents the current sheet at the surface. The thin solid line represents the path of an electron in the plasma below the surface, moving in the fields due to the current sheet, and being specularly reflected. The thin dashed line represents the path of a corresponding electron in a "phantom" plasma above the surface. Clearly, if the specularly reflecting barrier is removed, there is no change, the electrons above and below merely change places.

The prescription is therefore the following. In the infinite metal, with dielectric relation of the form (2.5), one solves the Maxwell equations with a plane current source of the form

$$
j(\boldsymbol{r}, t)=\boldsymbol{J} \delta(z) \exp [\mathrm{i}(\boldsymbol{p} \cdot \boldsymbol{\rho}-\omega t)], \quad \hat{z} \cdot \boldsymbol{J}=0 .
$$

The solution can be written

$$
\boldsymbol{E}(\boldsymbol{r}, t)=\boldsymbol{E}(z) \exp [\mathrm{i}(\boldsymbol{p} \cdot \boldsymbol{\rho}-\omega t)], \quad \boldsymbol{B}(\boldsymbol{r}, t)=\boldsymbol{B}(z) \exp [\mathrm{i}(\boldsymbol{p} \cdot \boldsymbol{\rho}-\omega t)],
$$

where

$$
\begin{aligned}
& \boldsymbol{E}(z)=\frac{2}{\mathrm{i} \omega} \int_{-\infty}^{\infty} \mathrm{d} q\left(\frac{\boldsymbol{J}-\boldsymbol{k} \cdot \boldsymbol{J} / \boldsymbol{k}^{2}}{\left(\varepsilon_{\mathrm{t}}(k, \omega)-c^{2} k^{2} / \omega^{2}\right.}+\frac{\boldsymbol{k} \cdot \boldsymbol{J}}{k^{2} \varepsilon_{r}(k, \omega)}\right) \mathrm{e}^{\mathrm{i} q z} \\
& \boldsymbol{B}(z)=\frac{2 c}{\mathrm{i} \omega^{2}} \int_{-\infty}^{\infty} \mathrm{d} q \frac{\boldsymbol{k} \times \boldsymbol{J}}{\varepsilon_{\mathrm{t}}(k, \omega)-c^{2} k^{2} / \omega^{2}} \mathrm{e}^{\mathrm{i} q z}
\end{aligned}
$$

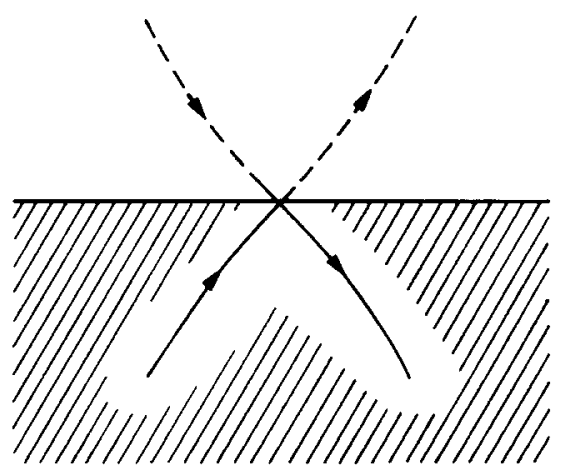

Fig. 1. Specular reflection trajectories for an electron and its image in the SCIB model. 
in which $\boldsymbol{k}=\boldsymbol{p}+q \hat{z}$ in the integrands. The surface impedances are then given by (2.19) using the fields (2.23) evaluated in the limit $z \rightarrow 0^{-}$. To form this limit we first note that for an isotropic medium $\varepsilon_{\ell}(k, \omega)$ and $\varepsilon_{t}(k, \omega)$ are even functions of $k$ and, hence, $q$ in the integrands of $(2.23)$. We can use this fact to replace the integrals over all $q$ by integrals over positive $q$ only. For the components of the electric field parallel to the interface, the resulting integral is well behaved, and one obtains the limit by simply setting $z=0$ in the integrand. The components of the magnetic field parallel to the interface are, however, proportional to the integral

$$
I \equiv \int_{-\infty}^{\infty} \mathrm{d} q \frac{q \mathrm{e}^{\mathrm{i} q z}}{\varepsilon_{\mathrm{t}}(k, \omega)-c^{2} k^{2} / \omega^{2}}=2 \mathrm{i} \int_{0}^{\infty} \mathrm{d} q \frac{q \sin q z}{\varepsilon_{\mathrm{t}}(k, \varpi)-c^{2} k^{2} / \omega^{2}}
$$

which appears to vanish in the limit $z \rightarrow 0^{-}$. But this is true only for the integral over any finite interval. For large $q$ we can use the fact that $\varepsilon_{\mathrm{t}}(\infty, \omega)$ is finite to write

$$
\lim _{z \rightarrow 0^{-}} I=-\frac{2 \mathrm{i} \omega^{2}}{c^{2}} \lim _{z \rightarrow 0^{-}} \int_{0}^{\infty} \mathrm{d} q \frac{\sin q z}{q}=\frac{\mathrm{i} \pi \omega^{2}}{c^{2}}
$$

With this one gets the result

$$
\begin{aligned}
& Z^{\mathrm{P}}(p, \omega)=\frac{8 \mathrm{i}}{\omega} \int_{0}^{\infty} \mathrm{d} q \frac{1}{k^{2}}\left(\frac{q^{2}}{\varepsilon_{\mathrm{t}}(k, \omega)-c^{2} k^{2} / \omega^{2}}+\frac{p^{2}}{\varepsilon_{\ell}(k, \omega)}\right), \\
& Z^{\mathrm{S}}(p, \omega)=\frac{8 \mathrm{i}}{\omega} \int_{0}^{\infty} \mathrm{d} q \frac{1}{\varepsilon_{\mathrm{t}}(k, \omega)-c^{2} k^{2} / \omega^{2}}, \quad \text { SCIB }
\end{aligned}
$$

where $k^{2}=p^{2}+q^{2}$ in the integrals.

Since the argument leading to the expressions (2.26) for the surface impedances relies upon the reflection symmetry of the classical Boltzmann equation, strictly speaking, the infinite medium dielectric constants obtained in the semiclassical approximation should be used in these expressions. These dielectric constants appear in many places; one of the clearest and most detailed discussions is that of Lindhard [9]. When the effects of collisions are taken into account in the single relaxation-time approximation the result can be written

$$
\begin{aligned}
& \varepsilon_{\ell}(k, \omega)=\varepsilon_{\mathrm{b}}(\omega)+\frac{3 \omega_{\mathrm{p}}^{2}}{\omega+\mathrm{i} \nu} \frac{u^{2} f_{\ell}(0, u)}{\omega+\mathrm{i} \nu f_{\ell}(0, u)}, \\
& \varepsilon_{\mathrm{t}}(k, \omega)=\varepsilon_{\mathrm{b}}(\omega)-\frac{\omega_{\mathrm{p}}^{2}}{\omega(\omega+\mathrm{i} \nu)} f_{\mathrm{t}}(0, u), \quad \text { (semiclassical) }
\end{aligned}
$$

in which $\varepsilon_{\mathrm{b}}$ is a background wavevector-independent dielectric constant associated with the bound 
electrons, $\dagger \nu$ is the electron collision frequency, $\omega_{\mathrm{p}}=\left(4 \pi n e^{2} / m\right)^{1 / 2}$ is the plasma frequency, and

$$
u=(\omega+\mathrm{i} \nu) / k v_{\mathrm{F}},
$$

with $v_{\mathrm{F}}$ the Fermi velocity. The functions $f_{\ell}$ and $f_{\mathrm{t}}$ are

$$
f_{\ell}(0, u)=1-\frac{1}{2} u \ln (u+1) /(u-1), \quad f_{t}(0, u)=\frac{3}{2} u^{2}-\frac{3}{2} u\left(u^{2}-1\right) \ln (u+1) /(u-1) .
$$

The redundant variable in $f_{\ell}$ and $f_{t}$, here set equal to zero, is present because these are in fact the semiclassical limits of functions introduced below for the quantum gas.

Although, to be completely consistent, the SCIB model should employ the semiclassical expressions (2.27) in the formulas (2.26) for the surface impedances, it has become customary to extend the model by using instead the quantum infinite medium dielectric constants. These are

$$
\begin{aligned}
& \varepsilon_{\ell}(k, \omega)=\varepsilon_{\mathrm{b}}(\omega)+\frac{3 \omega_{\mathrm{p}}^{2}}{\omega+\mathrm{i} \nu} \frac{u^{2} f_{\ell}(z, u)}{\omega+\mathrm{i} \nu f_{\ell}(z, u) / f_{\ell}(z, 0)}, \\
& \varepsilon_{\mathrm{t}}(k, \omega)=\varepsilon_{\mathrm{b}}(\omega)-\omega_{\mathrm{p}}^{2}\left\{\omega\left[f_{\mathrm{t}}(z, u)-3 z^{2} f_{\ell}(z, u)\right]+\mathrm{i} \nu\left[f_{\mathrm{t}}(z, 0)-3 z^{2} f_{\ell}(z, 0)\right]\right\} / \omega^{2}(\omega+\mathrm{i} \nu),
\end{aligned}
$$

where

$$
u=(\omega+\mathrm{i} \nu) / k v_{\mathrm{F}}, \quad z=k / 2 k_{\mathrm{F}},
$$

with $v_{\mathrm{F}}$ the Fermi velocity and $k_{\mathrm{F}}=m v_{\mathrm{F}} / \hbar$ the Fermi wavevector. The functions $f_{\ell}$ and $f_{\mathrm{t}}$ are

$$
\begin{aligned}
& f_{\ell}(z, u)=\frac{1}{2}+\frac{1-(z-u)^{2}}{8 z} \ln \frac{z-u+1}{z-u-1}+\frac{1-(z+u)^{2}}{8 z} \ln \frac{z+u+1}{z+u-1}, \\
& f_{\mathrm{t}}(z, u)=\frac{3}{8}\left(z^{2}+3 u^{2}+1\right)-3 \frac{\left[1-(z-u)^{2}\right]^{2}}{-32 z} \ln \frac{z-u+1}{z-u-1}-3 \frac{\left[1-(z+u)^{2}\right]^{2}}{\frac{32 z}{2 z}} \ln \frac{z+u+1}{z+u=1} .
\end{aligned}
$$

In (2.30) these functions with argument $u$ set equal to zero are to be interpreted as the limit as $u$ goes to zero through positive imaginary values, so that

$$
f_{\ell}(z, 0)=\frac{1}{2}+\frac{1-z^{2}}{4 z} \ln \frac{|1+z|}{|1-z|}, \quad f_{\mathrm{t}}(z, 0)=\frac{3}{8}\left(z^{2}+1\right)-3 \frac{\left(1-z^{2}\right)^{2}}{16 z} \ln \frac{|1+z|}{|1-z|} .
$$

The semiclassical limit corresponds to the limit $z \rightarrow 0$ in these expressions, in which case the expressions (2.30) reduce to the semiclassical expressions (2.27). In fact in many applications the quantity $z$ is small,

\footnotetext{
$\dagger$ It is well known but not widely explained that the bound and free electron response are additive and that there is no local field correction in a plasma. The best explanation still seems to be that given in the classic papers of Darwin [10].

$\ddagger$ The derivation of these formulas has a rather checkered history. They were first obtained by Lindhard [9] for the special case $\nu=0$ and $\varepsilon_{\mathrm{b}}=1$. Later Kliewer and Fuchs [11] attempted to include the effects of finite electron collision frequency by using constraints of physical consistency to guess the form. Unfortunately their conjectures were not quite correct. The correct form for the fongitudinal dielectric constant was given by Mermin [12], while that for the transverse case has only recently been obtained by one of us [13].
} 
and there is little reason to prefer quantum infinite medium expressions over the semiclassical expressions. The most important physical phenomenon not described properly by the semiclassical expressions is the sharp cutoff of electron-hole pair absorption at large wavevectors. We discuss this phenomenon in chapter 3 in connection with molecular fluorescence.

\subsection{The quasistatic approximation}

The quasistatic approximation to Maxwell's equations is discussed in most textbooks on electrodynamics as an approximation appropriate for finding the magnetic field due to currents varying slowly in time $[3,6]$. It is usually stated as an approximation in which the displacement current in Ampère's law is neglected. This statement, while appropriate for the applications envisioned in these texts, is not sufficiently general for our purposes. In fact, the quasistatic approximation arises from a systematic expansion of Maxwell's equations in powers of $c^{-1}$, and we exhibit this in the following paragraph. We then discuss the conditions of validity of the approximation, the essential point being that the fields at short distances from the source, the near fields, are always quasistatic. For phenomena involving small molecules placed close to metal surfaces, this will therefore be a useful approximation.

From the inhomogeneous Maxwell equations, i.e., Gauss's law and Ampère's law, one sees that for $c$ large, $\boldsymbol{D}$, and therefore $\boldsymbol{E}$, will be of zeroth order in $c^{-1}$, while $\boldsymbol{B}$ will be of first order. Keeping this in mind, the zeroth-order quasistatic equations are obtained by formally setting $c=\infty$ in Maxwell's equations (2.1). The result is

$$
\operatorname{curl} \boldsymbol{E}=0, \quad \operatorname{div} D=4 \pi \rho \quad \text { (quasistatic). }
$$

In next order the terms of order $c^{-1}$ are kept in Ampère's law, and the magnetic field is determined from

$$
\operatorname{div} \boldsymbol{B}=0, \quad \operatorname{curl} \boldsymbol{B}=\frac{4 \pi}{c}\left(j+\frac{1}{4 \pi} \frac{\partial \boldsymbol{D}}{\partial t}\right) \quad \text { (quasistatic) }
$$

in which it is understood that $\boldsymbol{D}(\boldsymbol{r}, t)$ is the solution of the zeroth-order equations. Stopping at this point, eqs. (2.34) and (2.35) determine the quasistatic fields. The quasistatic electric field is just the instantaneous Coulomb field due to the given charge distributions $\rho(r, t)$, while the quasistatic magnetic field is the instantaneous Biot-Savart field arising from the given current distribution $j(r, t)$ together with the displacement current arising from the quasistatic electric field.

The quasistatic approximation is appropriate in such a variety of physical situations that it is difficult to state in utmost generality the conditions of validity. The most important physical phenomenon neglected is radiation. The quasistatic fields are instantaneous as opposed to the retarded fields, and without retardation there is no radiation. Even in a radiating system, however, the near fields in the neighborhood of the system are quasistatic and can be evaluated with the neglect of retardation. In general, therefore, the quasistatic approximation is valid at distances from the source small compared with the wavelength of emitted radiation. To be more specific, for a confined charge-current system in vacuum, varying harmonically in time with frequency $\omega$, the fields are quasistatic at distances $r \ll c / \omega$.

The reflection coefficients in quasistatic approximation are particularly simple. First of all, there are no S-polarized waves, as we see from the fact that for such waves the ratio of $\boldsymbol{B}$ to $\boldsymbol{E}$ as given by (2.17) is of order $c$, not $c^{-1}$ as required by the quasistatic approximation. In fact the S-polarized waves emitted 
by a source are important only in the radiation zone, far from the source. In the near zone only the P-polarized waves are significant. The form of these P-polarized waves is given by (2.16) in which we replace $q_{1}$ by its quasistatic limit,

$$
q_{1} \rightarrow \mathrm{ip} \quad(c \rightarrow \infty) .
$$

Thus the quasistatic electric and magnetic fields in the local medium above the surface have the form

$$
\begin{aligned}
& \boldsymbol{E}(\boldsymbol{r})=-\frac{c p}{\omega \varepsilon_{1}} B_{\mathrm{P}}\left[(\hat{z}+\mathrm{i} \hat{\boldsymbol{p}}) \mathrm{e}^{p z}+r_{12}^{\mathrm{P}}(\hat{\boldsymbol{z}}-\mathrm{i} \hat{\boldsymbol{p}}) \mathrm{e}^{-p z}\right] \exp (\mathrm{i} \boldsymbol{p} \cdot \boldsymbol{\rho}), \\
& \boldsymbol{B}(\boldsymbol{r})=B_{\mathrm{P}} \hat{\boldsymbol{z}} \times \hat{\boldsymbol{p}}\left[\mathrm{e}^{p z}+r_{12}^{\mathrm{P}} \mathrm{e}^{-p z}\right] \exp (\mathrm{i} \boldsymbol{p} \cdot \boldsymbol{\rho}), \quad z>0 .
\end{aligned}
$$

If the medium below the surface, medium 2, is local, we find the quasistatic form of the reflection coefficient from (2.6) by replacing $q_{1}$ and $q_{2}$ by i $p$. Thus

$$
r_{12}^{\mathrm{P}}=\left(\varepsilon_{2}-\varepsilon_{1}\right) /\left(\varepsilon_{2}+\varepsilon_{1}\right) \quad(\text { local })
$$

Note that in the quasistatic approximation $r_{12}^{\mathrm{s}}$, given in (2.7) vanishes. For the SCIB model the quasistatic reflection coefficient is given by (2.00) with $q_{1} \rightarrow \mathrm{i} p$ and with $Z^{\mathrm{P}}$ given by the limit as $c \rightarrow \infty$ of (2.26). The result is

$$
r_{12}^{\mathrm{P}}=\left[1-\varepsilon_{1} \frac{2 p}{\pi} \int_{0}^{\infty} \mathrm{d} q \frac{1}{k^{2} \varepsilon_{\ell}(k, \omega)}\right] /\left[1+\varepsilon_{1} \frac{2 p}{\pi} \int_{0}^{\infty} \mathrm{d} q \frac{1}{k^{2} \varepsilon_{\ell}(k, \omega)}\right], \quad \mathrm{SCIB}
$$

with, as before, $k^{2}=p^{2}+q^{2}$ in the integrals.

We have stated these results as arising from $c \rightarrow \infty$ in (2.16). An alternative way of reaching the same results is to take $p \gg \omega / c$. Thus large $p$ corresponds to quasistatic behavior. This is not surprising since large $p$ corresponds to small distances.

\subsection{The quantum infinite barrier model}

In this section we consider an ideal quantum gas of electrons filling a half-space with an infinite potential barrier at the surface. We assume that the background dielectric constant is unity and that the electron collision frequency is vanishingly small. We derive the linear response of this system to electric fields in the quasistatic approximation. The result is the so-called quantum infinite barrier (QIB) model. This model was first discussed by Beck and Celli [14] and more recently by Persson and Persson [15] who call it the IBM model, and by Metiu and coworkers [16], who call it a jellium model with an infinite barrier. We repeat the discussion here with a slightly different formalism for a number of reasons: The discussion will allow us to exhibit the relation between the QIB and the SCIB models, to make clear how we numerically evaluate the results, and to correct a number of misprints which have appeared in the subsequent literature.

The quasistatic reflection coefficient for this model can be expressed in terms of the electric potential and its derivative just below the surface. To see this we express the electric field in the electron gas in terms of a potential of the form 


$$
V(\boldsymbol{r}, t)=V(z) \exp [\mathrm{i}(\boldsymbol{p} \cdot \boldsymbol{\rho}-\omega t)]
$$

Thus

$$
\boldsymbol{E}(\boldsymbol{r}, t)=-\operatorname{grad} V=-\left[\mathrm{i} \boldsymbol{p} V(z)+\hat{z} V^{\prime}(z)\right] \exp [\mathrm{i}(\boldsymbol{p} \cdot \boldsymbol{\rho}-\omega t)],
$$

where $V^{\prime}=\mathrm{d} V / \mathrm{d} z$. The components of the electric field parallel to the surface are continuous, therefore $\hat{\boldsymbol{p}} \cdot \boldsymbol{E}$ given by (2.37) and (2.41) must be equal, so that

$$
p V(0)=-\left(c p / \omega \varepsilon_{1}\right) B_{\mathrm{P}}\left(1-r_{12}^{\mathrm{P}}\right) \text {. }
$$

The component of the electric displacement perpendicular to the interface is continuous. Just above the surface, $\boldsymbol{D}=\varepsilon_{1} \boldsymbol{E}$, while just below, $\boldsymbol{D}=\boldsymbol{E}$, since the electron density vanishes at the infinite barrier. Hence,

$$
\{\hat{z} \cdot \boldsymbol{E}\}_{\text {inside }}=\varepsilon_{1}\{\hat{z} \cdot \boldsymbol{E}\}_{\text {outside }} .
$$

Using (2.41) and (2.37) this gives

$$
V^{\prime}(0)=(c p / \omega) B_{\mathrm{P}}\left(1+r_{12}^{\mathrm{P}}\right)
$$

Dividing this equation by (2.42) and solving the resulting equation for $r_{12}^{\mathrm{P}}$ we find

$$
r_{12}^{\mathrm{P}}=\frac{1-\varepsilon_{1} p V(0) / V^{\prime}(0)}{1+\varepsilon_{1} p V(0) / V^{\prime}(0)}
$$

which is our desired expression.

We see therefore that the reflection coefficient can be determined if the potential within the electron gas is known. To get an equation for $V(r, t)$, we use in the third Maxwell equation (2.1) the defining relation

$$
\boldsymbol{D}=\boldsymbol{E}+4 \pi \boldsymbol{P},
$$

where $\boldsymbol{P}$ is the polarization. Putting $\boldsymbol{E}=-\operatorname{grad} V$, we get

$$
\nabla^{2} V=-4 \pi\left(\rho+\rho_{\text {ind }}\right)
$$

where $\rho_{\text {ind }}(\boldsymbol{r}, t)=-\operatorname{div} \boldsymbol{P}$ is the induced charge density of the electron gas. This will be an equation for $V(r, t)$, if we can express $\rho_{\text {ind }}$ in terms of $V$, which we do below for an independent-particle model of the electron gas. $\dagger$

The quantum equation of motion for the one-particle density matrix is $\ddagger$

$\dagger$ The independent particle model is variously called the self-consistent field method by Kittel [17] or the random phase approximation by Pines [18]. A good introduction to the methods we employ is ref. [17] ch. 6 .

$\ddagger$ We use the same symbol for the density matrix, the charge density in Maxwell's equations, and the magnitude of $\rho$. This is the custom and which is meant should be clear from the context. 


$$
i \hbar \frac{\partial \rho}{\partial t}=\left[H_{0}-e V(r, t), \rho\right]
$$

where $H_{0}$ is the Hamiltonian for a free electron confined to the lower half-space by an infinite potential barrier

$$
H_{0}=-\frac{\hbar^{2}}{2 m} \nabla^{2}+ \begin{cases}\infty, & z>0 \\ 0, & z<0\end{cases}
$$

In discussing this equation we shall need the eigenstates of $H_{0}$. These are

$$
\psi_{\boldsymbol{k}_{\|}, \boldsymbol{k}_{\mathbf{z}}}(\boldsymbol{r})=\theta(-z) \exp \left(\boldsymbol{i}_{\|} \cdot \boldsymbol{\rho}\right) \sin k_{z} z
$$

where $\boldsymbol{r}=\boldsymbol{\rho}+z \hat{\boldsymbol{z}}$ and $\theta$ is the Heaviside function (equal to unity for positive argument and zero for negative argument). Here $\hat{z} \cdot \boldsymbol{k}_{\|}=0$ and $k_{z}>0$. The energy eigenvalues are

$$
H_{0} \psi_{\boldsymbol{k} \mid, k_{z}}=\mathscr{E}\left(\boldsymbol{k}_{\|}+k_{z} \hat{z}\right) \psi_{\boldsymbol{k} \mid, k_{z}}, \quad \mathscr{E}(\boldsymbol{k})=\hbar^{2} k^{2} / 2 m
$$

The orthogonality relation for these states is

$$
\begin{aligned}
\left\langle\psi_{\boldsymbol{k}_{\|}, \boldsymbol{k}_{z}}, \psi_{\boldsymbol{k}_{\|}^{\prime}, \boldsymbol{k}_{z}^{\prime}}\right\rangle & \equiv \int \mathrm{d} \boldsymbol{\rho} \int_{-\infty}^{0} \mathrm{~d} z \exp \left[\mathrm{i}\left(\boldsymbol{k}_{\|}-\boldsymbol{k}_{\|}^{\prime}\right) \cdot \boldsymbol{\rho}\right] \sin k_{z} z \sin k_{z}^{\prime} z \\
& =2 \pi^{3} \delta\left(\boldsymbol{k}_{\|}-\boldsymbol{k}_{\|}^{\prime}\right) \delta\left(k_{z}-k_{z}^{\prime}\right),
\end{aligned}
$$

and the completeness relation is

$$
\frac{1}{2 \pi^{3}} \int \mathrm{d} \boldsymbol{k}_{\|} \int_{0}^{\infty} \mathrm{d} k_{z} \psi_{\boldsymbol{k}_{\|,}, \boldsymbol{k}_{z}}(\boldsymbol{r}) \psi_{\boldsymbol{k}_{\|,}, \boldsymbol{k}_{z}}\left(\boldsymbol{r}^{\prime}\right)^{*}=\delta\left(\boldsymbol{r}-\boldsymbol{r}^{\prime}\right) \theta(-z)
$$

The trace of an operator $\mathcal{O}$ is

$$
\operatorname{Tr}\{\mathcal{O}\}=\frac{1}{2 \pi^{3}} \int \mathrm{d} \boldsymbol{k}_{\|} \int_{0}^{\infty} \mathrm{d} k_{z}\left\langle\psi_{\boldsymbol{k} \mid, \boldsymbol{k}_{z}}, \mathcal{O} \psi_{\boldsymbol{k}_{\mid k}, k_{z}}\right\rangle
$$

Another formula we shall use is the identity

$$
\exp (\mathrm{ip} \cdot \boldsymbol{\rho}) \psi_{\boldsymbol{k}_{\mathbb{l},}, \boldsymbol{k}_{z}}=\psi_{\boldsymbol{k}_{\|}+\boldsymbol{p}, \boldsymbol{k}_{z}} .
$$

In the absence of the electric potential $V$, the solution of $(2.48)$ is the equilibrium density matrix

$$
\rho_{0}=\left[\exp \left\{-\left(H_{0}-\mathscr{E}_{\mathrm{F}}\right) / k_{\mathrm{B}} T\right\}+1\right]^{-1},
$$


where $\mathscr{C}_{\mathrm{F}}$ is the Fermi energy, $T$ is the temperature, and $k_{\mathrm{B}}$ is the Boltzmann constant. The corresponding electron density is given by

$$
n\left(\boldsymbol{r}^{\prime}\right)=2 \operatorname{Tr}\left\{\delta\left(\boldsymbol{r}-\boldsymbol{r}^{\prime}\right) \rho_{0}\right\}
$$

where the factor 2 takes account of the spin degeneracy. Using (2.54) this becomes

$$
n(\boldsymbol{r})=\frac{1}{\pi^{3}} \int \mathrm{d} \boldsymbol{k}_{\|} \int_{0}^{\infty} \mathrm{d} k_{z} f(\mathscr{E})\left|\psi_{\boldsymbol{k} \|, \boldsymbol{k}_{z}}(\boldsymbol{r})\right|^{2},
$$

where $\mathscr{E}=\mathscr{E}\left(\boldsymbol{k}_{\|}+k_{z} \hat{z}\right)$, and

$$
f(\mathscr{E})=\left\{\exp \left[\left(\mathscr{E}-\mathscr{E}_{\mathrm{F}}\right) / k_{\mathrm{B}} T\right]+1\right\}^{-1}
$$

is the Fermi function. When $k_{\mathrm{B}} T \ll \mathscr{E}_{\mathrm{F}}$ the Fermi function can be approximated by its zero temperature or degenerate limit

$$
f(\mathscr{E})=\theta\left(\mathscr{E}_{\mathrm{F}}-\mathscr{E}\right), \quad k_{\mathrm{B}} T \ll \mathscr{E}_{\mathrm{F}} .
$$

In this limit the integral (2.58) is elementary. Using (2.50) we find

$$
n(r)=n_{0}\left[1-3 \frac{\sin 2 k_{\mathrm{F}} z}{\left(2 k_{\mathrm{F}} z\right)^{3}}+3 \frac{\cos 2 k_{\mathrm{F}} z}{\left(2 k_{\mathrm{F}} z\right)^{2}}\right] \theta(-z)
$$

where $k_{\mathrm{F}}=\left(\left(2 m \mathscr{E}_{\mathrm{F}}\right)^{1 / 2}\right) / \hbar$ is the Fermi wavevector and

$$
n_{0}=k_{\mathrm{F}}^{3} / 3 \pi^{2}
$$

is the equilibrium density of an infinite electron gas. In fig. 2 we show $n$, as given by (2.61), as a function

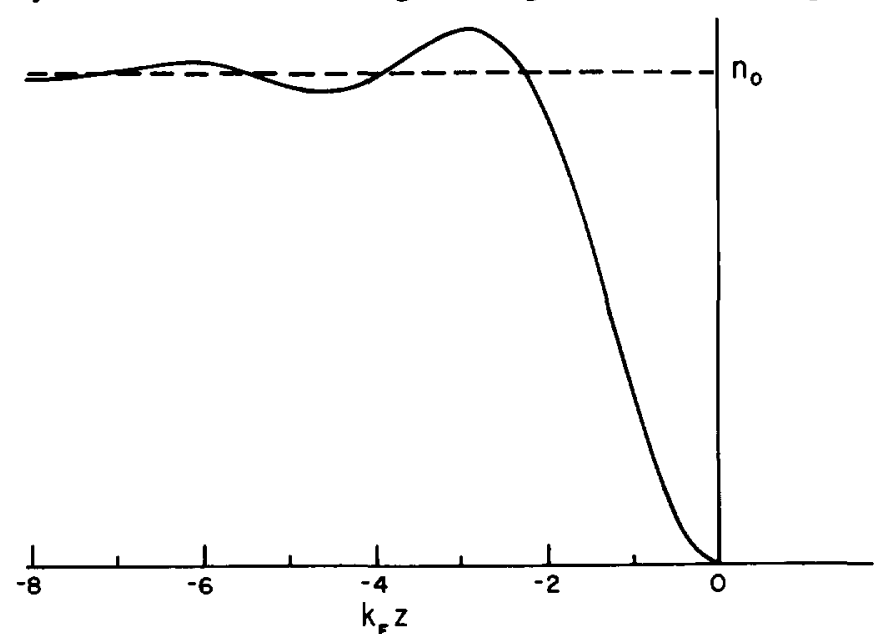

Fig. 2. Electron density profiles near the surface for the QIB (solid) and SCIB (dashed) models. 
of the depth below the surface. This figure shows clearly the difference between this QIB model and the corresponding SCIB model, for which $n$ would be equal to the constant $n_{0}$ all the way to the surface, where it would vanish abruptly. Probably the most significant aspect of this difference is that for the QIB model the density vanishes continuously. Probably less significant are the Friedel oscillations of $n$ as it approaches $n_{0}$ in the interior of the metal. From the figure we also conclude that in comparing the two models, we should probably take the barrier for the SCIB model somewhat inside that for the QIB model at, for example, the half-density point.

After these preliminaries, we return to the problem posed above: solving (2.48) to express the induced charge density in terms of $V$. We do this by seeking a perturbation solution of the form

$$
\rho(t)=\rho_{0}+\delta \rho \mathrm{e}^{-\mathrm{i} \omega t}
$$

where $\rho_{0}$ is the equilibrium density matrix (2.56) and $\delta \rho$ is the first-order term in $V$. Putting this in (2.48) and keeping only first-order terms we find

$$
\hbar \omega \delta \rho-\left[H_{0}, \delta \rho\right]=-e\left[V(z) \exp (\mathrm{ip} \cdot \boldsymbol{\rho}), \rho_{0}\right]
$$

where we have used (2.40). We form the matrix elements of this equation with respect to the eigenstates (2.50), using (2.51) and (2.55), to find

$$
\left(\hbar \omega+\mathscr{E}-\mathscr{E}^{\prime}\right)\left\langle\psi_{\boldsymbol{k}_{1}^{\prime}, k_{z}^{\prime}}, \delta \rho \psi_{\boldsymbol{k}_{\mid,}, \boldsymbol{k}_{z}}\right\rangle=-e\left[f(\mathscr{E})-f\left(\mathscr{E}^{\prime}\right)\right]\left\langle\psi_{\boldsymbol{k}_{\boldsymbol{k}}^{\prime}, k_{z}^{\prime}}, V(z) \psi_{\boldsymbol{k} \mid+\boldsymbol{p}, k_{z}}\right\rangle,
$$

where $\mathscr{E}=\mathscr{E}\left(\boldsymbol{k}_{\|}+k_{z} \hat{z}\right), \mathscr{E}^{\prime}=\mathscr{E}\left(\boldsymbol{k}_{\|}^{\prime}+k_{z}^{\prime} \hat{z}\right)$, and $f(\mathscr{E})$ is the Fermi function (2.60). The electron density variation is $\delta n(r, t)=\delta n(r) \mathrm{e}^{-\mathrm{i} \omega t}$, where as in (2.57),

$$
\begin{aligned}
\delta n\left(\boldsymbol{r}^{\prime}\right) & =2 \operatorname{Tr}\left\{\delta\left(\boldsymbol{r}-\boldsymbol{r}^{\prime}\right) \delta \rho\right\} \\
& =\left(1 / 2 \pi^{6}\right) \int \mathrm{d} \boldsymbol{k}_{\|} \int_{0}^{\infty} \mathrm{d} k_{z} \int \mathrm{d} \boldsymbol{k}_{\|}^{\prime} \int_{0}^{\infty} \mathrm{d} k_{z}^{\prime} \psi_{\boldsymbol{k}_{\|,}, \boldsymbol{k}_{z}}^{*}\left(\boldsymbol{r}^{\prime}\right) \psi_{\boldsymbol{k}_{k, k}^{\prime}, k_{z}^{\prime}}\left(\boldsymbol{r}^{\prime}\right)\left\langle\psi_{\boldsymbol{k}_{\|,}^{\prime}, \boldsymbol{k}_{z}^{\prime}}, \delta \rho \psi_{\boldsymbol{k}_{\|}, \boldsymbol{k}_{z}}\right\rangle,
\end{aligned}
$$

in which we have used the completeness relation (2.53). Solving (2.65) for the matrix element of $\delta \rho$, with the understanding that $\omega$ has a small positive imaginary part, we put the result in this last expression to get

$$
\delta n(\boldsymbol{r})=-\frac{\mathrm{e}}{2 \pi^{6}} \int \mathrm{d} \boldsymbol{k}_{\|} \int_{0}^{\infty} \mathrm{d} k_{z} \int \mathrm{d} \boldsymbol{k}_{\|}^{\prime} \int_{0}^{\infty} \mathrm{d} \boldsymbol{k}_{z}^{\prime} \frac{f(\mathscr{E})-f\left(\mathscr{E}^{\prime}\right)}{\mathscr{E}-\mathscr{E}^{\prime}+\hbar \omega}\left\langle\psi_{\boldsymbol{k}_{\|}^{\prime}, \boldsymbol{k}_{z}^{\prime}}, V(z) \psi_{\boldsymbol{k} \|+\boldsymbol{p}, \boldsymbol{k}_{z}}\right\rangle \psi_{\boldsymbol{k}_{\|}, \boldsymbol{k}_{z}}^{*}(\boldsymbol{r}) \psi_{\boldsymbol{k}_{\|,}^{\prime}, \boldsymbol{k}_{z}^{\prime}}(\boldsymbol{r})
$$

Using the form (2.50) of the eigenstates, we see that

$$
\left\langle\psi_{\boldsymbol{k}_{\|}^{\prime}, \boldsymbol{k}_{z}^{\prime}}, V(z) \psi_{\boldsymbol{k}_{\|}+\boldsymbol{p}, \boldsymbol{k}_{z}}\right\rangle=(2 \pi)^{2} \delta\left(\boldsymbol{k}_{\|}^{\prime}-\boldsymbol{k}_{\|}-\boldsymbol{p}\right) \int_{-\infty}^{0} \mathrm{~d} z^{\prime} \sin k_{z}^{\prime} z^{\prime} \sin k_{z} z^{\prime} V\left(z^{\prime}\right) .
$$

Putting this in (2.67) and identifying the induced charge density as $\rho_{\text {ind }}=-e \delta n$, we can write 


$$
\rho_{\text {ind }}(r, t)=\exp [\mathrm{i}(\boldsymbol{p} \cdot \boldsymbol{\rho}-\omega t)] \int_{-\infty}^{0} \mathrm{~d} z^{\prime} g\left(z, z^{\prime}\right) V\left(z^{\prime}\right)
$$

where

$$
g\left(z, z^{\prime}\right)=\frac{2 e^{2}}{\pi^{4}} \int \mathrm{d} k_{\|} \int_{0}^{\infty} \mathrm{d} k_{z} \int_{0}^{\infty} \mathrm{d} k_{z}^{\prime} \frac{f(\mathscr{C})-f\left(\mathscr{C}^{\prime}\right)}{\mathscr{C}-\frac{\mathscr{C}^{\prime}}{\mathscr{C}}+\hbar \infty} \sin k_{z}^{\prime} z \sin k_{z} z \sin k_{z}^{\prime} z^{\prime} \sin k_{z} z^{\prime}
$$

in which $\mathscr{E}=\mathscr{E}\left(\boldsymbol{k}_{\|}+k_{z} \hat{z}\right), \mathscr{E}^{\prime}=\mathscr{E}\left(\boldsymbol{k}_{\|}+\boldsymbol{p}+k_{z}^{\prime} \hat{z}\right)$. The relation (2.69) is the one we seek, expressing the induced charge density in terms of $V$.

When (2.69) is put in (2.47) we get, using (2.40) and the fact that the charge density $\rho$ is zero in the electron gas,

$$
V^{\prime \prime}(z)-p^{2} V(z)+4 \pi \int_{-\infty}^{0} \mathrm{~d} z^{\prime} g\left(z, z^{\prime}\right) V\left(z^{\prime}\right)=0
$$

which is a homogeneous integro-differential equation for $V(z)$. We are to solve this in the region $-\infty<z<0$ with the boundary condition $V(-\infty)=0$ and $V^{\prime}(0)$ given. Then the reflection coefficient is given by (2.45).

There are several ways to go about solving this equation; one of the more efficient is to introduce the Fourier cosine transform of $V, \dagger$

$$
v(q)=\int_{-\infty}^{0} \mathrm{~d} z \cos q z V(z), \quad V(z)=(2 / \pi) \int_{0}^{\infty} \mathrm{d} q \cos q z v(q)
$$

Multiplying (2.71) by $\cos q z$ and integrating we get

$$
\left(q^{2}+p^{2}\right) v(q)-4 \pi \int_{0}^{\infty} \mathrm{d} q^{\prime} \Gamma\left(q, q^{\prime}\right) v\left(q^{\prime}\right)=V^{\prime}(0)
$$

where the term on the right comes from integrating the first term in (2.71) twice by parts, and where

$$
\Gamma\left(q, q^{\prime}\right)=(2 / \pi) \int_{-\infty}^{0} \mathrm{~d} z \int_{-\infty}^{0} \mathrm{~d} z^{\prime} g\left(z, z^{\prime}\right) \cos q z \cos q^{\prime} z^{\prime}
$$

Putting the expression (2.70) for $g\left(z, z^{\prime}\right)$ in this integrand, we can exchange the order of integration and 
perform the $z$ and $z^{\prime}$ integrals. Before doing this, however, note that the integrand of $(2.70)$ is an even function of $k_{z}$ and of $k_{z}^{\prime}$, so we may extend those integrals over the full range $-\infty$ to $\infty$ and divide by 4 . Putting the resulting expression in (2.74) and using the result

$$
\begin{aligned}
(4 / \pi) \int_{-\infty}^{0} \mathrm{~d} z \cos q z \sin k_{z}^{\prime} z \sin k_{z} z= & \delta\left(q+k_{z}-k_{z}^{\prime}\right)+\delta\left(q-k_{z}+k_{z}^{\prime}\right) \\
& -\delta\left(q+k_{z}+k_{z}^{\prime}\right)-\delta\left(q-k_{z}-k_{z}^{\prime}\right),
\end{aligned}
$$

we get

$$
\begin{aligned}
\Gamma\left(q, q^{\prime}\right)= & \frac{e^{2}}{16 \pi^{3}} \int \mathrm{d} \boldsymbol{k}_{\|} \int_{-\infty}^{\infty} \mathrm{d} k_{z} \int_{-\infty}^{\infty} \mathrm{d} k_{z}^{\prime} \frac{f(\mathscr{E})-f\left(\mathscr{E}^{\prime}\right)}{\mathscr{E}-\mathscr{E}^{\prime}+\hbar \omega}\left[\delta\left(q+k_{z}-k_{z}^{\prime}\right)+\delta\left(q-k_{z}+k_{z}^{\prime}\right)-\delta\left(q+k_{z}+k_{z}^{\prime}\right)\right. \\
& \left.-\delta\left(q-k_{z}-k_{z}^{\prime}\right)\right]\left[\delta\left(q^{\prime}+k_{z}-k_{z}^{\prime}\right)+\delta\left(q^{\prime}-k_{z}+k_{z}^{\prime}\right)-\delta\left(q^{\prime}+k_{z}+k_{z}^{\prime}\right)-\delta\left(q^{\prime}-k_{z}-k_{z}^{\prime}\right)\right] .
\end{aligned}
$$

When the factors in square brackets are multiplied, we get 16 products of $\delta$-functions, four of which may be dropped because $q$ and $q^{\prime}$ are positive. By changing the sign of $k_{z}$ or $k_{z}^{\prime}$ in the integration, the remaining products can be arranged as a group of four, each of the form

$$
\delta\left(q+k_{z}-k_{z}^{\prime}\right) \delta\left(q^{\prime}+k_{z}-k_{z}^{\prime}\right)=\delta\left(q-q^{\prime}\right) \delta\left(q+k_{z}-k_{z}^{\prime}\right)
$$

a second group of four of the form

$$
\delta\left(q-k_{z}-k_{z}^{\prime}\right) \delta\left(q^{\prime}-k_{z}+k_{z}^{\prime}\right)=\frac{1}{2} \delta\left(k_{z}-\frac{1}{2}\left(q+q^{\prime}\right)\right) \delta\left(k_{z}^{\prime}-\frac{1}{2}\left(q-q^{\prime}\right)\right)
$$

and a third group of four of the same form as the second but with $k_{z}$ and $k_{z}^{\prime}$ interchanged. Therefore we can write

$$
\Gamma\left(q, q^{\prime}\right)=G(K, \omega) \delta\left(q^{\prime}-q\right)-(1 / 4 \pi) S\left(q, q^{\prime}\right),
$$

where

$$
G(K, \omega)=\frac{e^{2}}{4 \pi^{3}} \int \mathrm{d} \boldsymbol{k}_{\|} \int_{-\infty}^{\infty} \mathrm{d} k_{z} \frac{f(\mathscr{E})-f\left(\mathscr{E}^{\prime}\right)}{\frac{\mathscr{E}}{\mathscr{E}-\mathscr{C}+\hbar \omega}}
$$

in which $\mathscr{E}=\mathscr{E}(\boldsymbol{k})$ and $\mathscr{E}^{\prime}=\mathscr{E}(\boldsymbol{k}+\boldsymbol{K})$ with $\boldsymbol{k}=\boldsymbol{k}_{\|}+k_{z} \hat{z}$ and $\boldsymbol{K}=\boldsymbol{p}+q \hat{z}$, and where

$$
\begin{aligned}
S\left(q, q^{\prime}\right)= & \frac{e^{2}}{2 \pi^{2}} \int \mathrm{d} \boldsymbol{k}_{\|} \int_{-\infty}^{\infty} \mathrm{d} k_{z} \int_{-\infty}^{\infty} \mathrm{d} k_{z}^{\prime} \frac{f(\mathscr{E})-f\left(\mathscr{E}^{\prime}\right)}{\mathscr{E}-\mathscr{E}^{\prime}+\hbar \omega} \\
& \times\left[\delta\left(k_{z}-\frac{1}{2}\left(q+q^{\prime}\right)\right) \delta\left(k_{z}^{\prime}-\frac{1}{2}\left(q-q^{\prime}\right)\right)+\delta\left(k_{z}-\frac{1}{2}\left(q-q^{\prime}\right)\right) \delta\left(k_{z}^{\prime}-\frac{1}{2}\left(q+q^{\prime}\right)\right)\right],
\end{aligned}
$$

in which $\mathscr{E}=\mathscr{E}\left(\boldsymbol{k}_{\|}+k_{z} \hat{z}\right)$ and $\mathscr{E}^{\prime}=\mathscr{E}\left(\boldsymbol{k}_{\|}+\boldsymbol{p}+k_{z}^{\prime} \hat{z}\right)$. 
The quantity $G(K, \omega)$ is the Lindhard response function corresponding to a plane wave with wavevector $\boldsymbol{K}$ in an infinite electron gas. With $f(\mathscr{E})$ given by $(2.60)$, the integral $(2.80)$ is familiar $[9,18]$, and we can write

$$
G(K, \omega)=-\left(3 \omega_{\mathrm{p}}^{2} / 4 \pi v_{\mathrm{F}}^{2}\right) f_{\ell}(z, u),
$$

where $f_{\ell}(z, u)$ is the longitudinal Lindhard function $(2.32), v_{\mathrm{F}}=\hbar k_{\mathrm{F}} / m$ is the Fermi velocity, $\omega_{\mathrm{p}}=$ $\left(4 \pi n_{0} e^{2} / m\right)^{1 / 2}$ is the plasma frequency, and the dimensionless variables $u$ and $z$ are given by (2.31) with $\nu \rightarrow 0^{+}$. The integration in (2.81) is also elementary and we can write

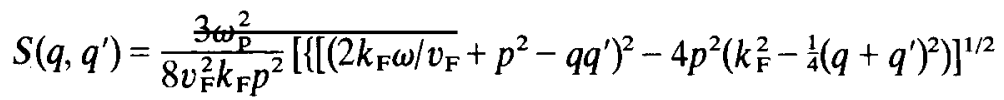

$$
\begin{aligned}
& \left.-\left[\left(2 k_{\mathrm{F}} \omega / v_{\mathrm{F}}-p^{2}+q q^{\prime}\right)^{2}-4 p^{2}\left(k_{\mathrm{F}}^{2}-\frac{1}{4}\left(q+q^{\prime}\right)^{2}\right)\right]^{1 / 2}-2\left(p^{2}-q q^{\prime}\right)\right\} \theta\left(k_{\mathrm{F}}-\frac{1}{2}\left(q+q^{\prime}\right)\right) \\
& +\left\{\left[\left(2 k_{\mathrm{F}} \omega / v_{\mathrm{F}}+p^{2}+q q^{\prime}\right)^{2}-4 p^{2}\left(k_{\mathrm{F}}^{2}-\frac{1}{4}\left(q-q^{\prime}\right)^{2}\right)\right]^{1 / 2}\right. \\
& \left.\left.\left.-\left[\left(2 k_{\mathrm{F}} \omega / v_{\mathrm{F}}-p^{2}-q q^{\prime}\right)^{2}-4 p^{2}\left(k_{\mathrm{F}}^{2}-\frac{1}{4}\left(q-q^{\prime}\right)^{2}\right)\right]^{1 / 2}-2\left(p^{2}+q q^{\prime}\right)\right\} \theta\left(k_{\mathrm{F}}-\frac{1}{2} \mid q-q^{\prime}\right)\right)\right] .
\end{aligned}
$$

In this expression the square roots are to be chosen on the branch with positive imaginary part. Here, as throughout this discussion, $\omega$ is to have a small (infinitesimal) positive imaginary part. Note, incidentally, that $S\left(q, q^{\prime}\right)=S\left(q^{\prime}, q\right)$, and that

$$
\int_{0}^{\infty} \mathrm{d} q^{\prime} S\left(q, q^{\prime}\right)=4 \pi G(K, \omega),
$$

which is a consequence of the fact that the induced charge density vanishes at the surface.

Putting (2.79) in (2.73) we get

$$
\left[K^{2}-4 \pi G(K, \omega)\right] v(q)+\int_{0}^{\infty} \mathrm{d} q^{\prime} S\left(q, q^{\prime}\right) v\left(q^{\prime}\right)=V^{\prime}(0),
$$

where $K^{2}=p^{2}+q^{2}$. With $G$ and $S$ given by the explicit expressions (2.82) and (2.83), this is an integral equation for $v(q)$. The reflection coefficient is given in terms of the solution of this equation by (2.47) with

$$
V(0)=(2 / \pi) \int_{0}^{\infty} \mathrm{d} q v(q) .
$$

We have solved this equation numerically by iteration. The initial, or beginning, iterate is obtained by dropping the integral in (2.85) to get

$$
v^{(0)}(q)=\left[K^{2}-4 \pi G(K, \omega)\right]^{-1} V^{\prime}(0) .
$$


The $n$th iterate is then given by

$$
v^{(n)}(q)=\left[K^{2}-4 \pi G(K, \omega)\right]^{-1}\left[V^{\prime}(0)-\int_{0}^{\infty} \mathrm{d} q^{\prime} S\left(q, q^{\prime}\right) v^{(n-1)}\left(q^{\prime}\right)\right]
$$

Typically we find convergence after 5-10 iterations. We then form $V(0)$ by numerical integration of (2.86) and form the reflection coefficient (2.45). In this connection we should point out for the benefit of anyone repeating these calculations that the numerical integration over $S$ is delicate, since the expression (2.83) has sharp structure corresponding to the branch points of the square roots.

The initial iterate (2.87) corresponds exactly to the SCIB model, as we see by putting $v^{(0)}$ in $(2.86)$ and forming the reflection coefficient with (2.45). The result is identical to the quasistatic SCIB result (2.39), providing we put $\varepsilon_{1}=1$ and make the connection

$$
\varepsilon_{\ell}(K, \omega)=1-4 \pi G(K, \omega) / K^{2},
$$

which with (2.80) is identical to $\varepsilon_{\ell}$ given by (2.30) when $\varepsilon_{\mathrm{b}}=1$ and $\nu \rightarrow 0^{+}$. Thus the SCIB model, for the case in which we neglect electron scattering and the bound-electron contribution to the dielectric constant, is obtained by dropping the integral term in (2.85).

In fig. 3 we show the real and imaginary parts of the quasistatic reflection coefficient plotted as
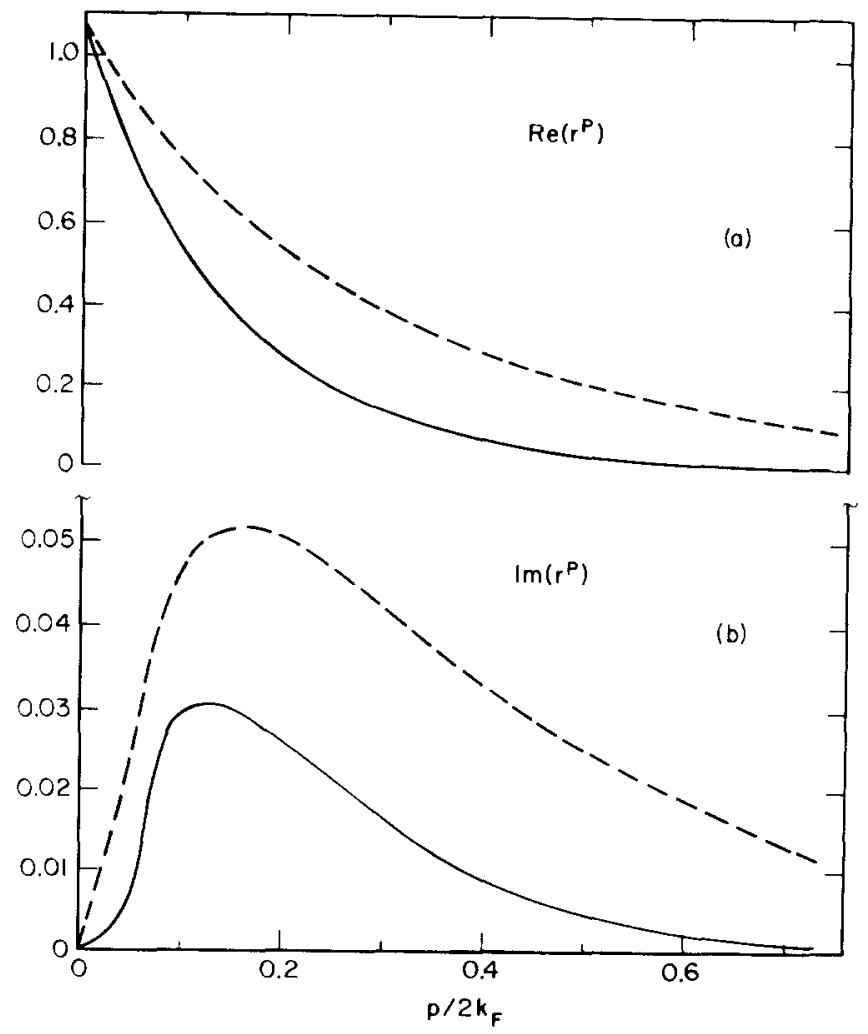

Fig. 3. Quasistatic reflection coefficient for a free electron gas with $\hbar \omega_{\mathrm{p}}=10.8 \mathrm{eV}$ at $\hbar \omega=1.96 \mathrm{eV}$ calculated for the QIB (solid) and SCIB (dashed) models. 
functions of $p$, the component of the wavevector parallel to the interface. The solid curves correspond to the QIB model, the dashed curves to the SCIB model. In both instances the parameters chosen were those appropriate to $\mathrm{Cu}$ in the visible region: $\hbar \omega_{\mathrm{p}}=10.8 \mathrm{eV}, \mathscr{E}_{\mathrm{F}}=7.0 \mathrm{eV}, \hbar \omega=1.96 \mathrm{eV}$, and, of course, $\varepsilon_{\mathrm{b}}=1, \nu=0$. The most obvious conclusion from these numerical results is that the two models give qualitatively similar predictions. Quantitatively both the real and imaginary parts of $r^{\mathrm{P}}$ are significantly smaller in the QIB model.

\subsection{Reflection in the long-wavelength limit}

In a considerable body of work Feibelman [19-22] has considered the reflection from a metal interface in the long-wavelength limit and applied the results to a number of problems, notably the problem of photoemission near the plasma frequency [20], and, of interest to us, the problem of the image field at an atom near the interface [21]. In our view the long-wavelength limit is not appropriate in problems involving molecules near the interface where wavelengths of the order of the molecular spacing or the molecular size are important. Nevertheless we review in this section the methods of Feibelman for two reasons: They have been successful when applied to appropriate problems, e.g., the photoemission problem, and they have been applied to problems of molecules near the surface. Our aim in this section will be first to give a simple exposition of the methods and then to relate them with the methods discussed earlier in the chapter.

First we should explain what is meant by the long-wavelength limit. This is the limit in which well within the metal the dielectric relation is of the local form. From our considerations earlier in this chapter, in particular from an inspection of the expressions (2.30) for the quantum infinite medium dielectric constants, we know that the local approximation requires first that the wavelength be long compared with the Fermi wavelength, or

$$
k \ll 2 k_{\mathrm{F}},
$$

and second that the phase velocity of the waves be large compared with the Fermi velocity, or

$$
k \ll \omega / v_{\mathrm{F}} .
$$

Since $\omega / 2 k_{\mathrm{F}} v_{\mathrm{F}}=\hbar \omega / 4 \mathscr{E}_{\mathrm{F}}$ is in general small, the latter is the operative requirement in our considerations. Under these conditions the dielectric relation in the region of the metal below a narrow surface region (of thickness some multiple of the Fermi wavelength) will be of the local form

$$
\boldsymbol{D}(\boldsymbol{r})=\varepsilon_{2}(\omega) \boldsymbol{E}(\boldsymbol{r}), \quad-z \gg v_{\mathrm{F}} / \omega
$$

On the other hand, within the surface region the dielectric relation will be nonlocal, of a form that for the moment will not be specified.

Now this limit, long-wavelength and a thin-surface region, is exactly that for which the Fresnel formulas (2.6) and (2.8) should be a very good approximation. And indeed they are, the corrections are small and in general negligible. An exception is near the plasma edge $\left(\omega \approx \omega_{\mathrm{p}}\right)$, where excitation of longitudinal plasma waves in the metal is significant, but even then the corrections to the Fresnel formulas are small, although experimentally important [20]. The object, therefore, will be to calculate the first-order corrections to the Fresnel formulas in this long-wavelength limit. The nominal expansion 
parameter will be the ratio of the thickness of the surface region to the wavelength. The starting point of our calculation will be the formulation in terms of surface impedances, introduced in section 2.1. Recall that the surface impedances can be calculated in terms of the solutions of the Maxwell equations within the metal alone, with appropriate boundary conditions at the metal surface.

Consider first the S-polarized case, for which within the metal

$$
\begin{aligned}
& \boldsymbol{E}(\boldsymbol{r})=E_{y}(z) \hat{z} \times \hat{\boldsymbol{p}} \exp (\mathrm{ip} \cdot \boldsymbol{\rho}), \quad \boldsymbol{D}(\boldsymbol{r})=D_{y}(z) \hat{z} \times \hat{\boldsymbol{p}} \exp (\mathrm{ip} \cdot \boldsymbol{\rho}) \\
& \boldsymbol{B}(\boldsymbol{r})=\left[B_{x}(z) \hat{\boldsymbol{p}}+B_{z}(z) \hat{z}\right] \exp (\mathrm{i} \boldsymbol{p} \cdot \boldsymbol{\rho}) .
\end{aligned}
$$

The Maxwell equations (2.2) then become

$$
\mathrm{d} E_{y} / \mathrm{d} z=-\mathrm{i}(\omega / c) B_{x}, \quad \mathrm{~d} B_{x} / \mathrm{d} z=-\mathrm{i}(c / \omega)\left[\left(\omega^{2} / c^{2}\right) D_{y}-p^{2} E_{y}\right]
$$

and

$$
B_{z}=(c p / \omega) E_{y}
$$

The surface impedance for S-polarized waves, as given in (2.19), is

$$
Z^{\mathrm{S}}=(4 \pi / c) E_{y}(0) / B_{x}(0)
$$

To form this quantity the procedure is to solve eqs. (2.94) for $B_{x}(z)$ within the metal $(-\infty<z<0)$ and with the boundary conditions that $E_{y}(0)$ is given and that $B_{x}(z)$ vanishes as $z \rightarrow-\infty$. We then form (2.96) with $B_{x}(0)$. In the local approximation where (2.92) holds throughout the metal, this solution is trivial. The result is

$$
E_{y}(z)=E_{y}(0) \exp \left(-\mathrm{i} q_{2} z\right), \quad B_{x}(z)=\left(c q_{2} / \omega\right) E_{y}(0) \exp \left(-\mathrm{i} q_{2} z\right) \quad \text { (local) }
$$

where $q_{2}=\left(\varepsilon_{2} \omega^{2} / c^{2}-p^{2}\right)^{1 / 2}$ with $\operatorname{Im} q_{2}>0$ as in (2.8). Forming (2.96) we find

$$
Z^{\mathrm{S}}=4 \pi \omega / c^{2} q_{2} \quad \text { (local) }
$$

which when put in (2.20) gives the Fresnel formula (2.7) for the S-polarized reflection coefficient.

To find the first-order correction to this local result we first integrate eqs. (2.94) to write

$$
E_{y}(0)=-\mathrm{i}(\omega / c) \int_{-\infty}^{0} \mathrm{~d} z B_{x}(z), \quad B_{x}(0)=-\mathrm{i}(c / \omega) \int_{-\infty}^{0} \mathrm{~d} z\left[\left(\omega^{2} / c^{2}\right) D_{y}(z)-p^{2} E_{y}(z)\right]
$$

We next multiply the first of these equations by $c q_{2} / \omega$ and subtract it from the second. After a little rearrangement using (2.8), we can write the result in the form

$$
B_{x}(0)=\frac{c q_{2}}{\omega} E_{y}(0)-\mathrm{i} \frac{\omega}{c} \int_{-\infty}^{0} \mathrm{~d} z\left[D_{y}(z)-\varepsilon_{2} E_{y}(z)\right]+\mathrm{i} q_{2} \int_{-\infty}^{0} \mathrm{~d} z\left[B_{x}(z)-\frac{c q_{2}}{\omega} E_{y}(z)\right] .
$$


Here the first term is the local result, as we see from (2.97), so that in the long-wavelength limit the integrals represent corrections to this result. Moreover, the factors multiplying these integrals are both inversely proportional to the wavelength, so that the corrections to first order in the ratio of surface thickness to wavelength are obtained by evaluating the integrals in the strictly infinite wavelength limit. $\dagger$ Now in this limit it is clear from eqs. (2.94) that $E_{y}$ and $B_{x}$ are uniform,

$$
E_{y}(z)=E_{y}(0), \quad B_{x}(z)=B_{x}(0)=\left(c q_{2} / \omega\right) E_{y}(0), \quad(\omega / c \rightarrow 0)
$$

This means that in the limit the second integral in (2.100) vanishes since the integrand is identically zero. The first integral does not vanish since the dielectric displacement corresponding to a uniform (but oscillating at frequency $\omega$ ) electric field is not uniform in the surface region. We are therefore led, following Feibelman, to introduce the quantity $\ddagger$

$$
d_{\|}(\omega)=\frac{1}{\left(\varepsilon_{1}-\varepsilon_{2}\right) E_{y}(0)} \int_{-\infty}^{0} \mathrm{~d} z\left[\bar{D}_{y}(z)-\varepsilon_{2} E_{y}(0)\right]
$$

where $\bar{D}_{y}(z)$ is the electric displacement corresponding to a uniform field $E_{y}(0)$ directed parallel to the interface. With this we see therefore that through first order (2.100) can be written

$$
B_{x}(0)=\left[\left(c q_{2} / \omega\right)+\mathrm{i}(\omega / c)\left(\varepsilon_{2}-\varepsilon_{1}\right) d_{\|}\right] E_{y}(0),
$$

and, therefore, putting this in (2.96) and keeping terms through first order

$$
Z^{\mathrm{S}}=\left(4 \pi \omega / c^{2} q^{2}\right)\left[1-\mathrm{i}\left(\omega^{2} / c^{2} q^{2}\right)\left(\varepsilon_{2}-\varepsilon_{1}\right) d_{\|}\right]
$$

Consider next the P-polarized case, for which within the metal

$$
\begin{aligned}
& \boldsymbol{E}(\boldsymbol{r})=\left[E_{x}(z) \hat{\boldsymbol{p}}+E_{z}(z) \hat{z}\right] \exp (\mathrm{i} \boldsymbol{p} \cdot \boldsymbol{\rho}), \quad \boldsymbol{B}(\boldsymbol{r})=B_{y}(z) \hat{\boldsymbol{z}} \times \hat{\boldsymbol{p}} \exp (\mathrm{ip} \cdot \boldsymbol{\rho}) \\
& \boldsymbol{D}(\boldsymbol{r})=\left[D_{x}(z) \hat{\boldsymbol{p}}+D_{z}(z) \hat{z}\right] \exp (\mathrm{i} \boldsymbol{p} \cdot \boldsymbol{\rho}) .
\end{aligned}
$$

The Maxwell equations (2.2) then become

$$
\mathrm{d} E_{x} / \mathrm{d} z=-\mathrm{i}\left(\omega^{2} / c^{2} p\right) D_{z}+\mathrm{i} p E_{z}, \quad \mathrm{~d} D_{z} / \mathrm{d} z=-\mathrm{i} p D_{x}
$$

and

$$
B_{y}=-(\omega / c p) D_{z}
$$

The surface impedance for P-polarized waves, as given by (2.19), is

\footnotetext{
$\dagger$ In the infinite-wavelength limit the quantities $\omega / c, q_{2}$, and $p$ are all taken as inversely proportional to the wavelength and therefore go to zero while ratios such as $c q_{2} / \omega$ are finite.

$¥$ Because we choose the metal to occupy the region of negative $z$ with the plane $z=0$ being a plane above which all metal properties (e.g. electron density) vanish, our $d_{\|}$is not exactly that of Feibelman, but differs by an added real constant.
} 


$$
Z^{\mathrm{P}}=-\frac{4 \pi}{c} \frac{E_{x}(0)}{B_{y}(0)}=\frac{4 \pi p}{\omega} \frac{E_{x}(0)}{D_{z}(0)}
$$

In a manner similar to that in the S-polarized case we form this quantity by solving eqs. (2.106) for $E_{x}(z)$ in the region $-\infty<z<0$ and with the boundary conditions that $D_{z}(0)$ is given and that $E_{x}(z)$ vanishes as $z \rightarrow-\infty$. We then form (2.108) with $E_{x}(0)$. Again, we do this first in the local approximation, where the solution is

$$
D_{z}(z)=D_{z}(0) \exp \left(\mathrm{i} q_{2} z\right), \quad E_{x}(z)=\left(q_{2} / p \varepsilon_{2}\right) D_{z}(0) \exp \left(-\mathrm{i} q_{2} z\right) \quad \text { (local) }
$$

where again $q_{2}$ is given by (2.8). Forming the surface impedance we find

$$
Z^{\mathrm{P}}=4 \pi q_{2} / \omega \varepsilon_{2} \quad(\text { local })
$$

Again, proceeding as in the S-polarized case, to find the first-order corrections to this local result, we first integrate eqs. (2.106) to obtain

$$
E_{x}(0)=\frac{1}{\mathrm{i} p} \int_{-\infty}^{0} \mathrm{~d} z\left[\frac{\omega^{2}}{c^{2}} D_{z}(z)-p^{2} E_{z}(z)\right], \quad D_{z}(0)=-\mathrm{i} p \int_{-\infty}^{0} \mathrm{~d} z D_{x}(z)
$$

Next multiply the second of these equations by $q_{2} / p \varepsilon_{2}$ and subtract it from the first. The result can be rearranged into the form

$$
\begin{aligned}
E_{x}(0)= & \frac{q_{2}}{p \varepsilon_{2}} D_{z}(0)-\mathrm{i} p \int_{-\infty}^{0} \mathrm{~d} z\left[\frac{1}{\varepsilon_{2}} D_{z}(z)-E_{z}(z)\right] \\
& +\mathrm{i} \frac{q_{2}}{\varepsilon_{2}} \int_{-\infty}^{0} \mathrm{~d} z\left[D_{x}(z)-\varepsilon_{2} E_{x}(z)\right]+\mathrm{i} q_{2} \int_{-\infty}^{0} \mathrm{~d} z\left[E_{x}(z)-\frac{q_{2}}{p \varepsilon_{2}} D_{z}(z)\right] .
\end{aligned}
$$

The factors multiplying the integrals in these expressions are each inversely proportional to the wavelength, while the first term corresponds to the Fresnel, or infinite wavelength, result. The first-order corrections to this result are therefore obtained by evaluating the integrals in the infinite wavelength limit. In this limit, as we see from eqs. (2.106), $E_{x}$ and $D_{z}$ are uniform

$$
E_{x}(z)=E_{x}(0)=\left(q_{2} / p \varepsilon_{2}\right) D_{z}(0), \quad D_{z}(z)=D_{z}(0)
$$

Here we see the strategy of the rearrangement resulting in (2.111), since there the third integral vanishes in the limit, its integrand being identically zero. The second integral is the same as the corresponding integral in the S-polarized case, there being no difference between $x$ and $y$ in the infinite wavelength limit. We can therefore express it in terms of $d_{\|}$given by (2.101) with $x$ replaced by $y$. The first integral in (2.111) is new. We are therefore led, again following Feibelman, to introduce the quantity 


$$
d_{\perp}(\omega)=\frac{1}{\left(1 / \varepsilon_{2}-1 / \varepsilon_{1}\right) \theta_{z}(0)} \int_{-\infty}^{0} \mathrm{~d} z\left[\frac{1}{\varepsilon_{2}} D_{z}(0)-\bar{E}_{z}(z)\right]
$$

where $\bar{E}_{z}(z)$ is the electric field corresponding to a uniform electric displacement $D_{z}(0)$. Collecting these results we see that through first order (2.111) can be written

$$
E_{x}(0)=\frac{q_{2}}{p \varepsilon_{2}} D_{z}(0)+\mathrm{i} p\left(\frac{1}{\varepsilon_{1}}-\frac{1}{\varepsilon_{2}}\right) d_{\perp} D_{z}(0)-\mathrm{i} q_{2}\left(1-\frac{\varepsilon_{1}}{\varepsilon_{2}}\right) d_{\|} E_{x}(0)
$$

Solving for $E_{x}(0)$ and putting the result in (2.107) we get

$$
Z^{\mathrm{P}}=\frac{4 \pi q_{2}}{\omega \varepsilon_{2}}\left[1+\mathrm{i}\left(\frac{1}{\varepsilon_{1}}-\frac{1}{\varepsilon_{2}}\right) \frac{\varepsilon_{2} p^{2} d_{1}-\varepsilon_{1} q_{2}^{2} d_{\|}}{q_{2}}\right]
$$

where again we have kept only first-order terms.

The S- and P-polarized reflection coefficients are now given by $(2.20)$ with the surface impedances given by (2.104) and (2.115). The result through first order can be written

$$
r_{12}^{\mathrm{s}}=\frac{q_{1}-q_{2}}{q_{1}+q_{2}}\left(1+2 \mathrm{i} q_{1} d_{\|}\right), \quad r_{12}^{\mathrm{P}}=\frac{q_{1} \varepsilon_{2}-q_{2} \varepsilon_{1}}{q_{1 \varepsilon_{2}}+q_{2} \varepsilon_{1}}\left(1+2 \mathrm{i} q_{1} \frac{\varepsilon_{1} q_{2}^{2} d_{\|}-\varepsilon_{2} p^{2} d_{1}}{\frac{\varepsilon_{1} q_{2}^{2}}{2} \varepsilon_{2} p^{2}}\right) .
$$

As an illustration consider the very simple model in which one takes the dielectric relation to be local throughout the metal but with a different, anisotropic, form in a layer of thickness $d$ at the surface. That is,

$$
D(r)= \begin{cases}\varepsilon_{x x}[E(r)-\hat{z} \cdot E(r) \hat{z}]+\varepsilon_{z z} \hat{z} \cdot E(r) \hat{z}, & -d<z<0 \\ \varepsilon_{2} E(r), & -\infty<z<-d\end{cases}
$$

According to (2.102), to form $d_{\|}$we are to solve for the electric displacement $D_{y}(z)$ corresponding to a uniform electric field $E_{y}(0)$ parallel to the interface ( $x y$ plane). The solution is trivial;

$$
D_{y}(z)= \begin{cases}\varepsilon_{x x} E_{y}(0), & -d<z<0 \\ \varepsilon_{2} E_{y}(0), & -\infty<z<-d\end{cases}
$$

With this (2.102) becomes

$$
d_{\|}=\left[\left(\varepsilon_{2}-\varepsilon_{x x}\right) /\left(\varepsilon_{2}-\varepsilon_{1}\right)\right] d .
$$

According to (2.113), to form $d_{\perp}$ we are to solve for the electric field $\boldsymbol{E}_{z}(z)$ corresponding to a uniform electric displacement perpendicular to the interface. Again the solution is trivial;

$$
\boldsymbol{E}_{z}(z)= \begin{cases}\left(1 / \varepsilon_{z z}\right) D_{z}(0), & -d<z<0 \\ \left(1 / \varepsilon_{2}\right) D_{z}(0), & -\infty<z<-d\end{cases}
$$

With this (2.113) becomes 


$$
d_{\perp}=\left[\left(1 / \varepsilon_{2}-1 / \varepsilon_{z z}\right) /\left(1 / \varepsilon_{2}-1 / \varepsilon_{1}\right)\right] d
$$

Note that if we choose $\varepsilon_{x x}=\varepsilon_{z z}=\varepsilon_{2}$, corresponding to a local metal with no surface layer, then $d_{\|}=d_{\perp}=0$ and from (2.116) we recover the Fresnel formulas. On the other hand, if we choose $\varepsilon_{x x}=\varepsilon_{z z}=\varepsilon_{1}$, corresponding also to a local metal with no surface layer, but with the surface now at $z=-d$, then $d_{\|}=d_{\perp}=d$. Again from (2.116) we recover the Fresnel formulas, but with the first approximation to the phase shift of the reflected wave due to propagation from $z=0$ to $z=-d$ and back. From these results we see that $d_{\|}$and $d_{\perp}$ can be interpreted as effective thicknesses of the surface region.

Next consider the SCIB and QIB models. Here we make the quasistatic approximation, for which the definitions (2.102) and (2.113) of $d_{\|}$and $d_{\perp}$ are unchanged, but in the formulas (2.104) and (2.115) for the surface impedances and in the formulas (2.116) for the reflection coefficients we make the replacements $q_{1} \rightarrow \mathrm{i} p$ and $q_{2} \rightarrow \mathrm{i} p$. In this approximation the S-polarized reflection coefficient vanishes, as expected, and the P-polarized reflection coefficient becomes

$$
r_{12}^{\mathrm{P}}=\frac{\varepsilon_{2}-\varepsilon_{1}}{\varepsilon_{2}+\varepsilon_{1}}\left(1-2 p \frac{\varepsilon_{1} d_{\Downarrow}+\varepsilon_{2} d_{1}}{\varepsilon_{1}+\varepsilon_{2}}\right)
$$

To form $d_{\|}$we are to calculate $D_{y}(z)$ corresponding to a uniform electric field $E_{y}(0)$ directed parallel to the interface and oscillating at frequency $\omega$. For these models the electron density $n(z)$ is unchanged by such a field, so the solution is

$$
D_{y}(z)=\left(1-4 \pi n(z) e^{2} / m \omega^{2}\right) E_{y}(0)
$$

The dielectric constant deep within the metal is $\left(\varepsilon_{\mathrm{b}}=1, \nu=0\right)$

$$
\varepsilon_{2}=1-4 \pi n_{0} e^{2} / m \omega^{2}=1-\omega_{\mathrm{p}}^{2} / \omega^{2}
$$

where $n_{0}=n(-\infty)$ is the asymptotic electron density. Putting these results in (2.102) we find

$$
d_{\|}=\frac{\varepsilon_{2}-1}{\varepsilon_{2}-\varepsilon_{1}} \int_{-\infty}^{0} \mathrm{~d} z \frac{n_{0}-n(z)}{n_{0}} .
$$

For the SCIB model $n(z)=n_{0}$ throughout the metal and $d_{\|}=0$. For the QIB model $n(z)$ is given by (2.61) and

$$
d_{\|}=\left[\left(\varepsilon_{2}-1\right) /\left(\varepsilon_{2}-\varepsilon_{1}\right)\right] 3 \pi / 8 k_{\mathrm{F}} \quad(\mathrm{QIB}) .
$$

Thus, for these models, aside from the factor $\left(\varepsilon_{2}-1\right) /\left(\varepsilon_{2}-\varepsilon_{1}\right)$, which is equal to unity if $\varepsilon_{1}=1$, the quantity $d_{\|}$is the "depth of the jellium edge", i.e., the depth at which a uniform positive background charge density $n_{0}$ would have to be terminated in order to ensure total charge neutrality.

Next consider the calculation of $d_{\perp}$ for the SCIB and QIB models. To form this quantity we are to calculate $E_{z}(z)$ corresponding to a uniform electric displacement $D_{z}(0)$ directed perpendicular to the interface and oscillating at frequency $\omega$. In the context of the discussion in section 2.3 , this means that 
for these models we are to calculate

$$
E_{z}(z)=-V^{\prime}(z)
$$

with

$$
D_{z}(0)=E_{z}(0)=-V^{\prime}(0)
$$

given. Here $V(z)$ is the potential corresponding to a uniform source in the $x y$ plane, i.e., it is the solution of (2.71) with $p=0$. Thus we can write (2.113) in the form

$$
d_{\perp}=\frac{1}{\left(1 / \varepsilon_{2}-1 / \varepsilon_{1}\right)} \int_{-\infty}^{0} \mathrm{~d} z\left[\frac{1}{\varepsilon_{2}}-\frac{V^{\prime}(z)}{V^{\prime}(0)}\right]
$$

In order to evaluate this expression in terms of the numerical results described in section 2.3 , it is convenient to express it in terms of the cosine transform of $V$. Thus, from (2.72), we write

$$
V^{\prime}(z)=-\frac{2}{\pi} \int_{0}^{\infty} \mathrm{d} q q v(q) \sin q z
$$

We put this in the second term in the integrand of (2.129) while in the first term we use the identity

$$
\frac{2}{\pi} \int_{0}^{\infty} \mathrm{d} q \frac{\sin q z}{q}=-1, \quad z<0
$$

to write

$$
d_{\perp}=\frac{1}{1 / \varepsilon_{2}-1 / \varepsilon_{1}} \int_{-\infty}^{0} \mathrm{~d} z \frac{2}{\pi} \int_{0}^{\infty} \mathrm{d} q\left[-\frac{1}{q \varepsilon_{2}}+\frac{q v(q)}{W^{\prime}(0)}\right] \sin q z
$$

Interchanging the order of integration and performing the $z$ integration we get

$$
d_{\perp}=\frac{1}{1 / \varepsilon_{2}-1 / \varepsilon_{1}} \frac{2}{\pi} \int_{0}^{\infty} \mathrm{d} q \frac{1}{q^{2}}\left(\frac{1}{\varepsilon_{2}}-\frac{q^{2} v(q)}{V^{\prime}(0)}\right)
$$

This is to be evaluated with $v(q)$ the solution of $(2.85)$ in which $p=0$. Note that for this solution

$$
\lim _{q \rightarrow 0} q^{2} v(q)=V^{\prime}(0) / \varepsilon_{2}
$$

with $\varepsilon_{2}$ the bulk dielectric constant (2.124). The integral (2.133) is therefore convergent. For the SCIB 
model we use (2.87), which with (2.89) gives the result

$$
d_{\perp}=\frac{1}{1 / \varepsilon_{2}-1 / \varepsilon_{1}} \frac{2}{\pi} \int_{0}^{\infty} \mathrm{d} q \frac{1}{q^{2}}\left(\frac{1}{\varepsilon_{2}}-\frac{1}{\varepsilon_{\ell}(q, \omega)}\right), \quad(\mathrm{SCIB})
$$

where $\varepsilon_{\ell}$ is the longitudinal dielectric constant (2.30) specialized to $\varepsilon_{\mathrm{b}}=1$ and $\nu=0$. For the QIB model we must evaluate (2.132) with the numerical solution of $(2.85)$.

Numerical results of these calculations for the example of a free electron gas with electron density appropriate to $\mathrm{Cu}$ in the visible region are shown in table 1. On first inspection the difference between the two models is quite large. However, if we were to choose the "surface" for the SCIB model to be at the depth of the jellium edge, which would correspond to adding $3 \pi / 8 k_{\mathrm{F}}=0.866 \AA$ to both $d_{\|}$and $d_{\perp}$ for that mode, the real parts are brought into very close agreement. There still remains a large discrepancy in the imaginary parts of $d_{\perp}$, but this is also misleading. Referring to the equation (2.122) for the reflection coefficient we see that $\operatorname{Im}\left(d_{\perp}\right)$ is proportional to the slope at the origin of $\operatorname{Im}\left(r^{\mathrm{P}}\right)$ shown in fig. 3. The slopes at the origin are indeed very different for the two models, but this is true only over a very narrow range of $p$, the slope for the QIB model quickly rises to be comparable to that for the SCIB model. We conclude once again that the two models give qualitatively similar predictions, and that quantitatively there is little difference, given that the effective position of the surface is an adjustable parameter in the SCIB model.

The fact that $d_{\|}$and $d_{\perp}$ are related only to the slope at the origin of the reflection coefficient as a function of $p$ makes this whole discussion appear to be rather trivial, since for the SCIB and QIB models it is no more difficult to calculate the whole curve as exemplified in fig. 3. Such a conclusion is misleading, however, since the calculation of $d_{\|}$and $d_{\perp}$ can be carried out for more realistic models of the electron density near the surface, models for which calculations of the sort described in the previous section would be impracticable. The way this is done is to replace the infinite barrier potential in the QIB Hamiltonian (2.49) by a more general potential $U(z)$ which is large and positive outside the metal and vanishes well inside the metal, with a smooth transition between. The result in the eigenstates (2.50) is to replace $\sin k_{z} z$ with $\phi_{k_{z}}(z)$, the corresponding eigenfunction in the one-dimensional potential $U(z)$, normalized so that well inside the metal $\phi_{k_{z}}(z) \sim \sin \left(k_{z} z+\delta\right)$. (The possible existence of bound states in the potential $U(z)$, corresponding to surface electronic states, is an additional complication.) The discussion in the remainder of section 2.3 goes through as before with the result that one obtains the expression (2.70) for the linear response function $g\left(z, z^{\prime}\right)$, but with the above replacement: $\sin k_{z} z \rightarrow$ $\phi_{k_{z}}(z)$. Calculations based on a formalism equivalent to this are reported by Feibelman in refs. [19-22].

Table 1

Numerical values of $d_{\|}$and $d_{1}$ for the SCIB and QIB models, calculated for a collisionless free electron gas with the same parameters as used in fig. 3

\begin{tabular}{lll}
\hline & $d_{\mathfrak{l}}(\AA)$ & $d_{\perp}(\AA)$ \\
\hline SCIB & 0 & $0.680-0.092 \mathrm{i}$ \\
QIB & 0.866 & $1.500-0.014 \mathrm{j}$ \\
\hline
\end{tabular}




\section{Molecular fluorescence near a metal}

In this chapter we address the problem of the emission of radiation by a molecule near a metal surface. Our primary interest will be in fluorescence, i.e., the decay of an excited electronic state of a molecule, but our results will be equally applicable to the final radiation step in a Raman or Rayleigh scattering process. The fluorescence problem has been reviewed by Chance et al. [23], and some of our discussion will repeat theirs. However, we will also cover two aspects of the problem which require further elucidation: the emission into surface plasmons and other guided modes, and the importance of specific mechanisms such as interband absorption, electron scattering loss, or electron-hole excitations in determining the fluorescence decay rate when the molecule is very close to the metal. The last two of these mechanisms can only be described accurately using a nonlocal response function for the metal.

The presence of a metal surface can markedly influence the fluorescence properties of a molecule nearby, and the nature of this influence depends on the distance $d$ from the metal. At distances of the order of or slightly greater than the emission wavelength $\left(d \geq 10^{3} \AA\right)$, the emission is primarily into photons and the fluorescent lifetime exhibits an oscillatory behavior with $d$, which arises from an interference between the directly radiated emission and that being reflected from the metal. This phenomenon was studied in the early experiments by Drexhage $[24,25]$ and was discussed theoretically by several authors [26-29]. At distances of order $d=50-500 \AA$, i.e., much larger than atomic dimensions but somewhat less than the wavelength, the fluorescing molecule couples strongly to the propagating surface plasmons on the metal $[30,31]$. This decay into surface plasmons has been observed directly in experiments which use prism or grating couplers to convert these surface waves into photons [32-36]. At very small values of $d(\leq 10 \AA)$, nonradiative processes dominate the decay rate, and these are determined by the intrinsic loss mechanisms in the metal: interband absorption, electron scattering losses, and electron-hole excitations. Which of these mechanisms is most important in a given experiment depends in detail upon the properties of the metal at the emission frequency. Only a few experiments have probed the small distance range [37,38].

The surface plasmon emission, although often termed "nonradiative" since it is usually not directly observable, is actually radiative in character since no loss in the metal is required and the emission is into propagating modes which have well-defined dispersion relations. The same is true of the emission into other types of guided modes which occur in multilayered structures.

To describe photon and surface plasmon emission, which dominate at large distances from the metal, the full retarded solutions of Maxwell's equations are required, but the metal response can be treated in local approximation. On the other hand, to describe the decay processes occurring at small distances from the metal, the quasistatic electromagnetic fields can be used, but a nonlocal metal response is required.

These fluorescence phenomena can all be understood in terms of a model of an oscillating point dipole above the surface. This problem was first discussed in the radio-frequency regime by Sommerfeld $[39,40]$ in the context of a radiating dipole above the conducting earth. Sommerfeld's solution was extended by Chance et al. [23] and others [26-31] for the purpose of explaining the optical fluorescence experiments. The solutions to this problem have usually been obtained by introducing the Hertz vector potential or by using dyadic Green's functions [23]. In our discussion we develop an alternative approach, originally due to Weyl [41], in which the dipolar field is written as a superposition of P- and $\mathrm{S}$-polarized plane waves and the influence of the metal surface is described by its plane wave reflection coefficients. This approach has been used by several authors [42-45]. It has the advantage of separating the calculation of the metal response, as described by the reflection coefficients, from the rest of the 
problem. It has the additional advantage that the solutions for problems with multiple interfaces can be obtained easily from the solution for a single interface.

\subsection{Point dipole above a semi-infinite metal}

In this section we derive a formula, expressed in terms of an integral involving the reflection coefficients, for the power dissipated by an oscillating dipole above a metal surface. To do this we first consider a dipole in the medium in the absence of the metal. Then, by a simple modification of the fields in accordance with the results of chapter 2 , we obtain the desired formula for the power dissipated in the presence of the metal.

Consider an oscillating point dipole $\boldsymbol{\mu} \mathrm{e}^{-\mathrm{i} \omega t}$ placed at $\boldsymbol{r}=\hat{z} d$ with arbitrary orientation in an infinite medium of real dielectric constant $\varepsilon_{1}$. The power dissipated by this dipole is given by

$$
\mathscr{P}=\omega / 2 \operatorname{Im}\left(\boldsymbol{\mu}^{*} \cdot \boldsymbol{E}\right)
$$

where $\boldsymbol{E} \mathrm{e}^{-\mathrm{i} \omega t}$ is the electric field at the dipole produced by the dipole, i.e., by the current source

$$
j(\boldsymbol{r}, t)=-\mathrm{i} \omega \boldsymbol{\mu} \mathrm{e}^{-\mathrm{i} \omega t} \delta(\boldsymbol{r}-\hat{z} d)
$$

To determine $\boldsymbol{E}$ we solve the Maxwell equations (2.2) with $\boldsymbol{D}=\varepsilon_{1} \boldsymbol{E}$ and this current source.

We introduce Fourier expansions of the fields

$$
\boldsymbol{E}(\boldsymbol{r})=\int \mathrm{d} \boldsymbol{k} \exp (\mathrm{i} \boldsymbol{k} \cdot \boldsymbol{r}) \boldsymbol{E}_{\boldsymbol{k}}, \quad \boldsymbol{B}(\boldsymbol{r})=\int \mathrm{d} \boldsymbol{k} \exp (\mathrm{i} \boldsymbol{k} \cdot \boldsymbol{r}) \boldsymbol{B}_{\boldsymbol{k}}
$$

then eqs. (2.2) can be written in the form

$$
\boldsymbol{B}_{k}=\frac{c}{\omega} \boldsymbol{k} \times \boldsymbol{E}_{k}, \quad \boldsymbol{k} \times \boldsymbol{B}_{k}+\varepsilon_{1} \frac{\omega}{c} \boldsymbol{E}_{k}=-\frac{\omega}{2 \pi^{2} c} \boldsymbol{\mu} \exp \left(-\mathrm{i} k_{z} d\right) .
$$

Solving these equations for the electric field amplitude, which is all we need, we find

$$
\boldsymbol{E}_{k}=\frac{-1}{2 \pi^{2} \varepsilon_{1}}\left\{\boldsymbol{\mu}+\frac{\boldsymbol{k} \times(\boldsymbol{k} \times \boldsymbol{\mu})}{k^{2}-k_{1}^{2}}\right\} \exp \left(-i k_{z} d\right)
$$

where $k_{1}^{2}=\varepsilon_{1} \omega^{2} / c^{2}$.

When this result is put in (3.3) we obtain an integral expression for $\boldsymbol{E}(\boldsymbol{r})$. Although at this point we are still dealing with the problem of the dipole in an infinite medium, our aim is to use the results in the problem in the presence of a metal surface, which can be taken to be the $x y$ plane. We therefore in the $\boldsymbol{k}$ integration in (3.3) write $\boldsymbol{k}=\boldsymbol{p}+\boldsymbol{k}_{z} \hat{z}$. The electric field then becomes

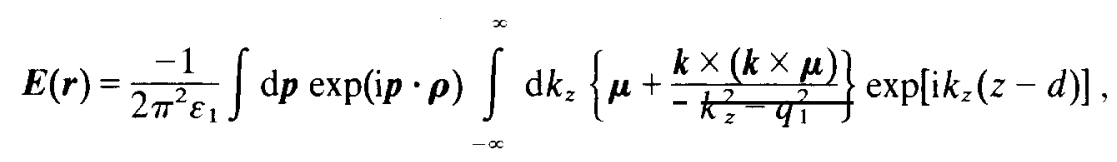


where we have written $\boldsymbol{r}=\boldsymbol{\rho}+z \hat{\boldsymbol{z}}$ and where

$$
q_{1}=\left(k_{1}^{2}-p^{2}\right)^{1 / 2}, \quad \operatorname{Im}\left(q_{1}\right) \geq 0
$$

The $k_{z}$ integration can be done using contour integration in the $k_{z}$ plane. The integrand in (3.6) has poles at $k_{z}= \pm q_{1}$. For $z>d$ we complete the contour in the upper half-plane; the integral is $2 \pi \mathrm{i}$ times the residue at $k_{z}=q_{1}$. For $z<d$ we complete the contour in the lower half-plane; the integral is $-2 \pi \mathrm{i}$ times the residue at $k_{z}=-q_{1}$. For $z=d$ there is an additional contribution to the integral for the $\boldsymbol{E}$ field equal to $2 \pi \delta(z-d)$ times the limit as $\left|k_{z}\right| \rightarrow \infty$ of the quantity in curly brackets. The result is

$$
\boldsymbol{E}(\boldsymbol{r})=\frac{-1}{\pi \varepsilon_{1}} \int \mathrm{d} \boldsymbol{p} \exp (\mathrm{ip} \cdot \boldsymbol{\rho})\left\{\delta(z-d) \hat{z} \cdot \boldsymbol{\mu} \hat{z}+\frac{\mathrm{i}}{2 q_{1}} \boldsymbol{k}_{1} \times\left(\boldsymbol{k}_{1} \times \boldsymbol{\mu}\right) \exp \left[\mathrm{i} q_{1}|z-d|\right]\right\}
$$

where

$$
\boldsymbol{k}_{1}=\boldsymbol{p}+q_{1} \hat{z}(z-d) /|z-d|
$$

To evaluate the dissipated power we insert (3.8) into (3.1). The singular $\delta$-function in the electric field does not contribute to the dissipated power since it corresponds to a real term in $\boldsymbol{\mu}^{*} \cdot \boldsymbol{E}$. The remaining terms are imaginary only for $0 \leq p \leq k_{1}$, i.e., only in the radiation regime. Remembering that at the dipole $\rho=0$ and $z=d$, we find after integrating over directions of $p$

$$
\mathscr{P}=\frac{\omega}{2 \varepsilon_{1}} \int_{0}^{k_{1}} \mathrm{~d} p \frac{p}{q_{1}}\left[p^{2} \mu_{\perp}^{2}+\frac{1}{2}\left(k_{1}^{2}+q_{1}^{2}\right) \mu_{\|]}^{2}\right] ;
$$

where we have introduced $\mu_{\perp}=|\hat{z} \cdot \boldsymbol{\mu}|$ and $\mu_{\|}=|\boldsymbol{\mu}-\hat{\boldsymbol{z}} \cdot \boldsymbol{\mu} \hat{z}|$. The $p$-integration is now elementary and we obtain the well-known Larmor formula for the power radiated by a dipole:

$$
\mathscr{P}=|\boldsymbol{\mu}|^{2} \varepsilon_{1}^{1 / 2} \omega^{4} / 3 c^{3}
$$

We now consider the extension of this result to the case when a metal (designated medium 2) is present in the region $z<0$. To find the electric field at the dipole we first decompose the field (3.8) in the region below the dipole into its $\mathrm{P}$ - and S-polarized components. This is accomplished using the vector identity

$$
-k_{1} \times\left(k_{1} \times \mu\right)=\left(p \hat{z}+q_{1} \hat{p}\right) \cdot \mu\left(p \hat{z}+q_{1} \hat{p}\right)+k_{1}^{2} \hat{z} \times \hat{p} \cdot \mu \hat{z} \times \hat{p}
$$

Using this in (3.8) the field emitted from the dipole toward the metal can be written

$$
\boldsymbol{E}_{\text {dipole }}(z<d)=\frac{\mathrm{i}}{2 \pi \varepsilon_{1}} \int \mathrm{d} \boldsymbol{p} \frac{\exp (\mathrm{i} \boldsymbol{p} \cdot \boldsymbol{\rho})}{q_{1}}\left\{\left(p \hat{z}+q_{1} \hat{\boldsymbol{p}}\right) \cdot \boldsymbol{\mu}\left(p \hat{z}+q_{1} \hat{\boldsymbol{p}}\right)+k_{1}^{2} \hat{z} \times \hat{\boldsymbol{p}} \cdot \boldsymbol{\mu} \hat{z} \times \hat{\boldsymbol{p}}\right\} \exp \left[-\mathrm{i} q_{1}(z-d)\right],
$$

where we have used the subscript "dipole" to emphasize that this is the downward propagating part of 
the field, coming from the dipole. The total field in the region $0<z<d$ must be this dipole field plus an upward propagating reflected field, with its components chosen so that the total field is a superposition of P- and S-polarized waves of the form (2.16) and (2.17), respectively. The first term in the brackets is a P-polarized wave, the second an S-polarized wave. The total field in this region is therefore

$$
\begin{aligned}
\boldsymbol{E}_{\mathrm{tot}}= & \frac{\mathrm{i}}{2 \pi \varepsilon_{1}} \int \mathrm{d} \boldsymbol{p}{\stackrel{\exp \left[\mathrm{i}\left(\boldsymbol{p} \cdot \boldsymbol{\rho}+q_{1} d\right)\right]}{q_{\mathrm{T}}}\left\{\boldsymbol{\mu} \cdot\left(p \hat{\boldsymbol{z}}+q_{1} \hat{\boldsymbol{p}}\right)\left[\left(p \hat{\boldsymbol{z}}+q_{1} \hat{\boldsymbol{p}}\right) \exp \left(-\mathrm{i} q_{1} z\right)+\left(p \hat{\boldsymbol{z}}-q_{1} \hat{\boldsymbol{p}}\right) r_{12}^{\mathrm{p}} \exp \left(\mathrm{i} q_{1} z\right)\right]\right.}+k_{1}^{2} \boldsymbol{\mu} \cdot \hat{\boldsymbol{z}} \times \hat{\boldsymbol{p}} \hat{\boldsymbol{z}} \times \hat{\boldsymbol{p}}\left[\exp \left(-\mathrm{i} q_{1} z\right)+\boldsymbol{r}_{12}^{\mathrm{s}} \exp \left(\mathrm{i} q_{1} z\right]\right\}, \quad 0<z<d
\end{aligned}
$$

The power dissipated by the dipole in the presence of the metal can now be evaluated by inserting (3.14) for $\boldsymbol{E}$ in (3.1) (remembering that the singular part of the dipole field gives no contribution to $\mathscr{P}$ ). After integrating over the directions of $p$, we obtain

$$
\begin{aligned}
\mathscr{P}= & \frac{\omega}{2 \varepsilon_{1}} \operatorname{Re} \int_{0}^{\infty} \mathrm{d} p \frac{p}{q_{1}}\left\{\mu_{\perp}^{2} p^{2}\left[1+r_{12}^{\mathrm{P}} \exp \left(2 \mathrm{i} q_{1} d\right)\right]+\frac{1}{2} \mu_{\|}^{2} k_{1}^{2}\left[1+r_{12}^{\mathrm{s}} \exp \left(2 \mathrm{i} q_{1} d\right)\right]\right. \\
& \left.+\frac{1}{2} \mu_{\|}^{2} q_{1}^{2}\left[1-r_{12}^{\mathrm{P}} \exp \left(2 \mathrm{i} q_{1} d\right)\right]\right\} .
\end{aligned}
$$

Here $\mu_{\|}$and $\mu_{\perp}$ are, respectively, the components of $\boldsymbol{\mu}$ parallel and perpendicular to the surface. This result, which is the main result of this section, is the generalization of the formula (3.10), to which it reduces when the reflection coefficients are set equal to zero.

The corresponding formula for the total power dissipated by a magnetic dipole $m$ in the same dielectric (nonmagnetic) medium above the metal is obtained by making the following replacements in (3.15): $\boldsymbol{\mu} \rightarrow \boldsymbol{m} ; \boldsymbol{r}_{12}^{\mathrm{P}} \rightarrow \boldsymbol{r}_{12}^{\mathrm{S}} ; \boldsymbol{r}_{12}^{\mathrm{S}} \rightarrow \boldsymbol{r}_{12}^{\mathrm{P}} ; \varepsilon_{1} \rightarrow 1$.

\subsection{Power dissipated spectrum}

The power dissipated (3.15) is expressed as an integral over the magnitude of a surface wave vector $\boldsymbol{p}$. For purposes of discussion it is convenient to introduce a quantity $\mathrm{d} \mathscr{P} / \mathrm{d} p$ defined as the $p$-space power spectrum, writing

$$
\mathscr{P}=\int_{0}^{\infty} \mathrm{d} p(d \mathscr{P} / \mathrm{d} p) .
$$

Different regions of this $p$ integration in (3.23) can be identified with different power dissipation channels. In this section we discuss the various contributions, showing that we can to a good approximation write the power dissipated as a sum

$$
\mathscr{P}=\mathscr{P}_{\text {photon }}+\mathscr{P}_{\text {SP }}+\mathscr{P}_{\text {LSW }}
$$

where $\mathscr{P}_{\text {photon }}$ is the power radiated via photons, $\mathscr{P}_{\text {SP }}$ via surface plasmons, and $\mathscr{P}_{\text {LSw }}$ that via what we call lossy surface waves.

We show in fig. 4 the $p$-space power spectrum at two different distances from the metal for the case 


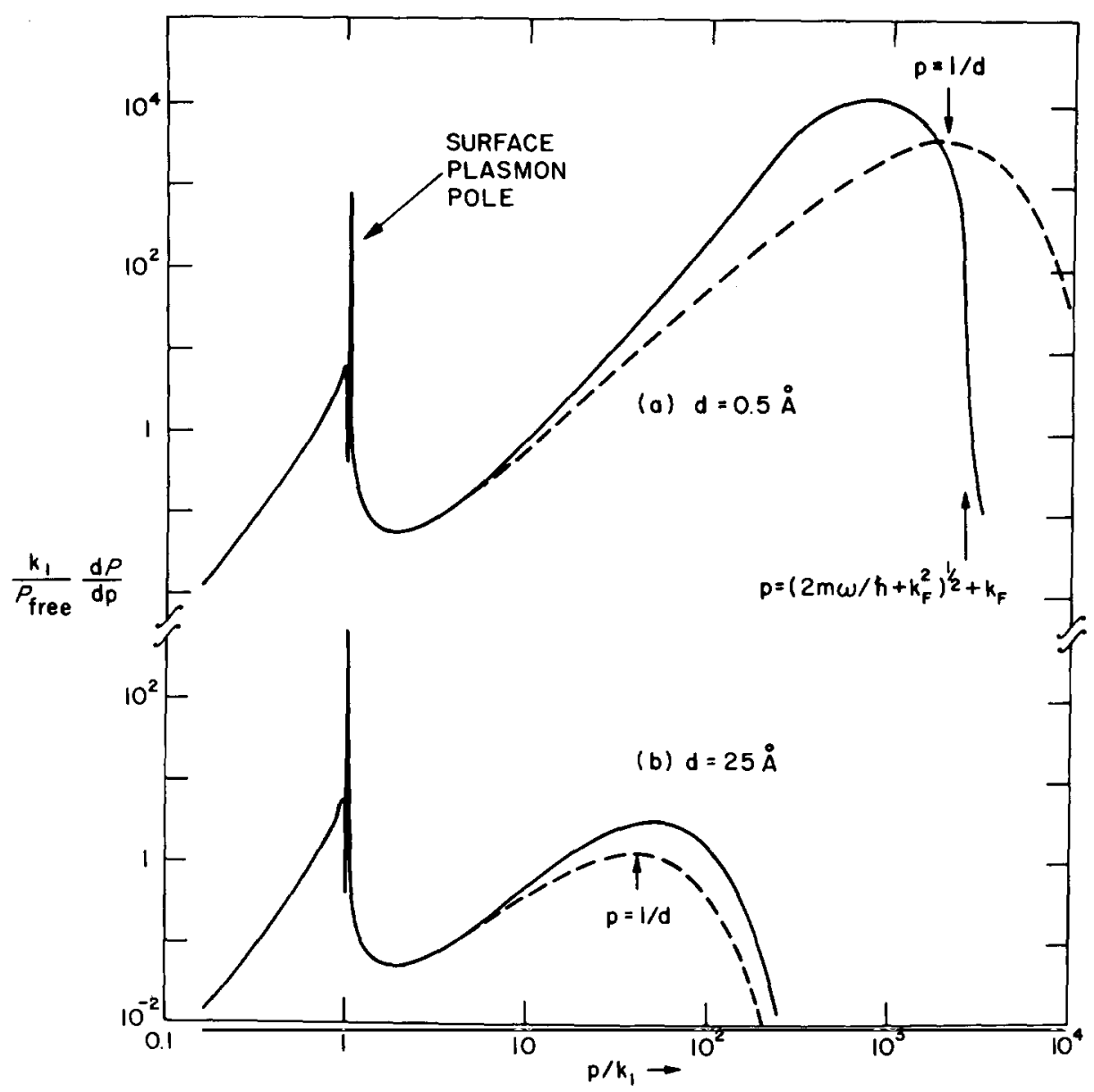

Fig. 4. The $p$-space power spectrum at $\hbar \omega=1.96 \mathrm{eV}$ for a $\frac{1}{\circ}$ dipole in vacuum outside a Ag surface calculated using the nonlocal SCIB model (solid) and the local model (dashed). In (a) the dipole is $0.5 \AA$ from the surface, in (b) it is $25 \AA$ away. The Ag parameters are given in the text.

of a dipole oriented normal to the surface and calculated for two different models of the metal. The parameters chosen apply to $\mathrm{Ag}$ at $\hbar \omega=1.96 \mathrm{eV}$. The solid curves are calculated with the nonlocal SCIB model in which $r_{12}^{\mathrm{P}}$ is given by (2.22) and (2.27) with the dielectric constants given by (2.31); the dashed curves are calculated with the local model in which $r_{12}^{\mathrm{P}}$ is given by (2.6). Both calculations employ the same interband term $\varepsilon_{\mathrm{b}}$, plasma frequency $\omega_{\mathrm{p}}$, and electron scattering rate $\nu . \dagger$

In the region $0 \leq p \leq k_{1}$ the $z$-component of the propagation vector $q_{1}$ is real, which means the waves in medium 1 are propagating bulk waves. We thus assign this part of the power spectrum to photon emission

$$
\mathscr{P}_{\text {photon }}=\int_{0}^{k_{1}} \mathrm{~d} p(\mathrm{~d} \mathscr{P} / \mathrm{d} p) .
$$

† The determination of the metal parameters is to some extent a matter of choice. In this case we have chosen to fit the optical data for Ag in the range $450-700 \mathrm{~nm}$ to obtain $\hbar \omega_{\mathrm{p}}=9.33 \mathrm{eV}, \hbar \nu=0.058 \mathrm{eV}$, and $\varepsilon_{\mathrm{b}}=3.6$ [46]. The remaining parameters were determined assuming one electron per atom and a spherical Fermi surface with the free-electron mass, giving $k_{\mathrm{F}}=\left(3 n \pi^{2}\right)^{1 / 3}=1.2 \times 10^{8} \mathrm{~cm}^{-1}$ and $v_{\mathrm{F}}=\hbar k_{\mathrm{F}} / m=1.4 \times 10^{8} \mathrm{~cm} / \mathrm{s}$. Note that we have fitted $\omega_{\mathrm{P}}$ as independent of $k_{\mathrm{F}}$ and $v_{\mathrm{F}}$, while in the free electron model they are related. 
Just above the light line, $p \geq k_{1}$, there is narrow peak in $\mathrm{d} \mathscr{P} / \mathrm{d} p$ which corresponds to surface plasmon emission. The peak arises from a pole in $r_{12}^{\mathrm{P}}$ at the complex surface plasmon wavevector $p_{\mathrm{SP}}$, which, using the local approximation (2.6), is given by

$$
p_{\mathrm{SP}}=(\omega / c)\left[\varepsilon_{1} \varepsilon_{2} /\left(\varepsilon_{1}+\varepsilon_{2}\right)\right]^{1 / 2} \text {. }
$$

The power emitted into surface plasmons can be determined by expanding $r_{12}^{\mathrm{P}}$ in the vicinity of this pole,

$$
r_{12}^{\mathrm{P}}\left(p \cong p_{\mathrm{SP}}\right)=\frac{2 \varepsilon_{1} \varepsilon_{2}}{\varepsilon_{1}^{2}-\varepsilon_{2}^{2}} \frac{p_{\mathrm{SP}}}{p-p_{\mathrm{SP}}}
$$

and then evaluating the pole contribution to (3.15). The result is

$$
\mathscr{P}_{\mathrm{SP}}=\frac{\pi c^{2}}{\omega} \operatorname{Re}\left\{\frac{\left(-\varepsilon_{2}\right)^{1 / 2} p_{\mathrm{SP}}^{5}}{\varepsilon_{1}^{3 / 2}\left(\varepsilon_{1}-\varepsilon_{2}\right)}\left(\mu_{\perp}^{2}-\frac{\varepsilon_{1}}{2 \varepsilon_{2}} \mu_{\|}^{2}\right) \exp \left(-2\left(-\varepsilon_{1} / \varepsilon_{2}\right)^{1 / 2} p_{\mathrm{SP}} d\right)\right\}
$$

Near the surface plasmon pole $p>k_{1}$ and $q_{1}=\left(k_{1}^{2}-p^{2}\right)^{1 / 2}$ is pure imaginary, corresponding to evanescent surface waves. This means the energy is trapped on the metal. However, the emission process is still radiative in nature, since the energy is carried away by a wave propagating along the surface. Moreover, no loss in the metal is required for this process to occur. In a multilayered structure several guided modes may exist in addition to, or instead of, the surface plasmon mode discussed here. Each guided mode of such a structure will produce a pole in $\mathrm{d} \mathscr{P} / \mathrm{d} p$ near the real $p$-axis. The power emitted into each can be evaluated by determining the pole contribution to (3.15) in a manner analogous to the above procedure for the surface plasmon pole. This will be shown in sections 3.4 and 3.5 below.

When we consider the large- $p$ region of fig. 4 we see there is a broad peak in $\mathrm{d} \mathscr{P} / \mathrm{d} p$. It is only in this region that there is a disparity between the local and nonlocal results. On the other hand, in this region of large $p$ the quasistatic approximation is valid. The formula for the power dissipated in this approximation can be obtained from (2.15) by setting $q_{1}=\mathrm{i} p$, as in (2.37), and $r_{12}^{\mathrm{s}}=0$.

$$
\mathscr{P}_{\text {LSW }}=\frac{\omega}{2 \varepsilon_{1}} \int_{0}^{\infty} \mathrm{d} p p^{2}\left(\mu_{\perp}^{2}+\frac{1}{2} \mu_{\|}^{2}\right) \mathrm{e}^{-2 p d} \operatorname{Im}\left(r_{12}^{\mathrm{P}}\right), \quad \text { (quasistatic) }
$$

Here we have ascribed the power dissipated to lossy surface waves, since in the large- $p$ region the dissipation arises from the imaginary part of the metal response and the fields which couple to the metal are evanescent waves associated with the near field of the dipole. The solid curves in fig. 4 are well fit in the region $p \gg k$ by $\mathrm{d} \mathscr{P} / \mathrm{d} p$ obtained from (3.22) with $r_{12}^{\mathrm{P}}$ given by (2.40), corresponding to the quasistatic SCIB model. Similarly, the dashed curves in fig. 4 are given quite accurately by using the local quasistatic result $r_{12}^{\mathrm{P}}=\left(\varepsilon_{2}-\varepsilon_{1}\right) /\left(\varepsilon_{2}+\varepsilon_{1}\right)$. In this case the integral in (3.22) can be evaluated to obtain

$$
\mathscr{P}_{\mathrm{LSW}}=\left(\omega / 8 \varepsilon_{1} d^{3}\right)\left(\mu_{\perp}^{2}+\frac{1}{2} \mu_{\|}^{2}\right) \operatorname{Im}\left[\left(\varepsilon_{2}-\varepsilon_{1}\right) /\left(\varepsilon_{2}+\varepsilon_{1}\right)\right] \quad \text { (local, quasistatic) }
$$

In the region $10 k_{1} \leq p \leq 10^{3} k_{1} \cong 2 k_{\mathrm{F}}$ of fig. 4 the nonlocal $\mathrm{d} \mathscr{P} / \mathrm{d} p$ exceeds the local by about an 
order of magnitude. This is a consequence of the fact that the local calculation does not describe electron-hole excitation, which dominates electron scattering loss in the high- $p$ region. At even larger $p$ values, as seen in fig. 4(a), the nonlocal result has a sharp cutoff beyond which it falls orders of magnitude below the local result. The cutoff corresponds to the upper edge of the bulk excitation spectrum. The fact that beyond the cutoff the nonlocal result falls so dramatically is a consequence of screening of the electron scattering loss mechanism. At large wavevectors this mechanism becomes ineffective, due to screening, as a means of absorbing energy from the electromagnetic fields.

The similarity of the appearance of the solid and dashed curves in the region of large $p$ is misleading. One might be tempted to think that the local calculation describes the effects of electron scattering while the nonlocal calculation describes in addition electron-hole excitation. While this picture has some validity at $p \ll 2 k_{\mathrm{F}}$, at larger values electron scattering is screened and is very much overestimated in a local calculation. The fact is that in the regions where the two calculations differ the local calculation is incorrect. On the other hand, in the low- $p$ region where the two calculations agree, the local calculation is obviously adequate.

There are, however, situations for which the region of validity of the local calculation is extended. One is for a rather poorer metal, such as liquid $\mathrm{Hg}$ where the electron scattering rate is about 20 times as large as that in $\mathrm{Ag}$. Then the power dissipated through electron scattering will dominate and the local and nonlocal calculations will coincide until at very large $p$ the nonlocal calculation will fall off faster due to screening. Another situation would be for a transition metal such as $\mathrm{Ni}$, for which the bound-electron dielectric constant $\varepsilon_{\mathrm{b}}$ has a large imaginary part, due to interband absorption. If interband absorption dominates, then the local calculation will be valid through very large $p$, there being no screening of $\varepsilon_{\mathrm{b}}$ by the electron gas. There will, however, be nonlocal effects in $\varepsilon_{\mathrm{b}}$ itself at extremely large wavevector.

The form of the $p$-space power spectrum in the LSW region is particularly simple for the local model. In this case $\mathrm{d} \mathscr{P} / \mathrm{d} p$ rises as $p^{2}$, has a peak at $p=1 / d$, and falls thereafter as $\exp (-2 p d)$. The form of $\mathrm{d} \mathscr{P} / \mathrm{d} p$ for the nonlocal model is more complicated and can best be discussed by referring to the dispersion relations for bulk modes in the metal, shown in fig. 5(a), and for modes associated with the vacuum-metal interface, shown in fig. 5(b). The propagating modes in the bulk metal are the transverse photon mode and the longitudinal plasmon mode, both occurring for $\omega>\omega_{\mathrm{p}}$, which is well above the range of frequencies of interest in our discussion. The other feature of the bulk mode spectrum is the broad continuum of electron-hole excitations, which occur at any given frequency for values of the bulk propagation vector $k$ in the range

$$
\left(2 m \omega / \hbar+k_{\mathrm{F}}^{2}\right)^{1 / 2}-k_{\mathrm{F}}<k<\left(2 m \omega / \hbar+k_{\mathrm{F}}^{2}\right)^{1 / 2}+k_{\mathrm{F}} .
$$

The modes associated with the interface are shown in fig. 5(b), where the abscissa is the component of the propagation vector parallel to the surface. The photon modes above the metal form a continuum, $0<p<k_{1}$, coming from the projection of the light line onto the interface. The continuum of electron-hole excitations is projected into a still broader continuum, $0<p<\left(2 m \omega / \hbar+k_{\mathrm{F}}^{2}\right)^{1 / 2}+k_{\mathrm{F}}$, with the same upper limit as the bulk excitations (3.29). In addition there is the propagating surface plasmon mode already discussed. The projections of the modes above $\omega_{p}$ are not shown since we are concerned with lower frequencies.

The radiating dipole couples to the surface excitations by matching the surface Fourier components in its fields with the surface excitation spectrum of fig. 2(b). The thin horizontal line in fig. 5(b) represents the path of integration in (3.15) at a frequency comparable to that used in fig. 4 . The photon 

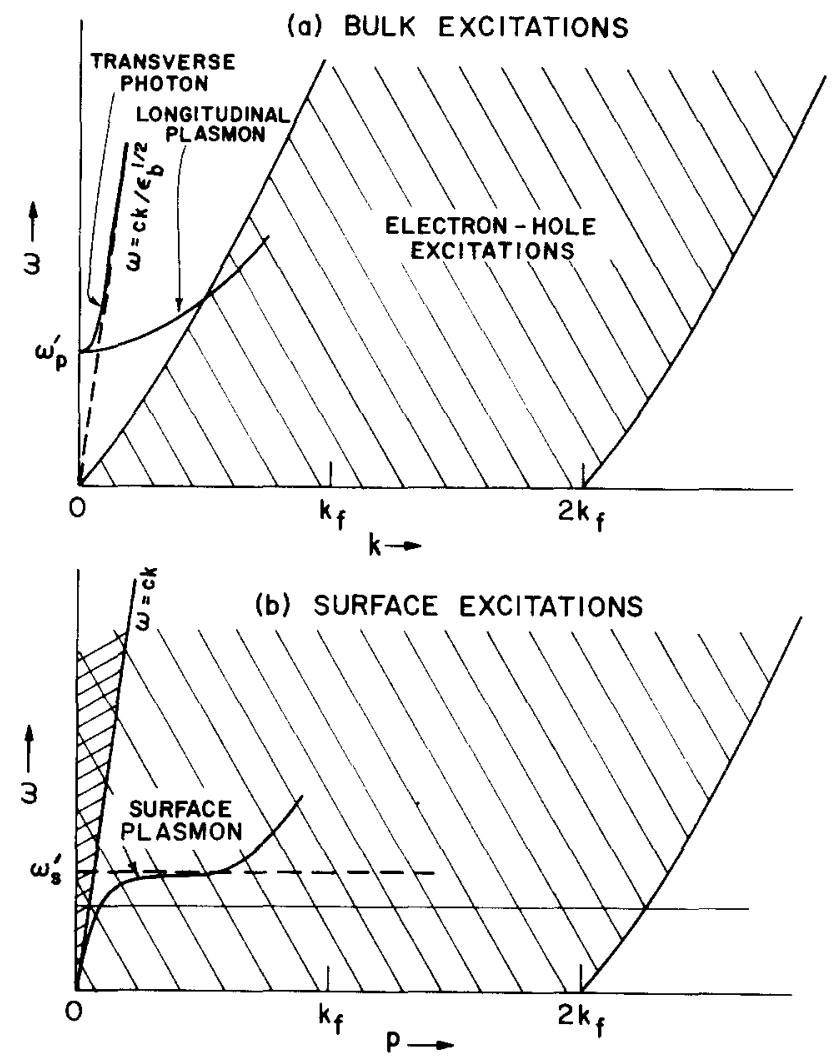

Fig. 5. Schematic excitation spectrum of a free-electron metal with a core polarization term $\varepsilon_{\mathrm{b}}$. In (a), $\omega_{\mathrm{p}}^{\prime}=\varepsilon_{\mathrm{b}}^{-1 / 2} \omega_{\mathrm{p}}$ is the bulk plasmon frequency. In (b), $\omega_{\mathrm{s}}^{\prime}=\left(\varepsilon_{\mathrm{b}}+1\right)^{-1 / 2} / \omega_{\mathrm{p}}$ is the asymptotic surface plasmon frequency for a local metal. The medium above the metal is vacuum. The thin horizontal line in (b) corresponds to the abscissa in fig. 4 . After ref. [42].

emission corresponds to the range $0<p<k_{1}$, to the left of the light line. The surface plasmon emission corresponds to the point on the dispersion curve just to the right of the light line. Finally, there is the broad underlying continuum of electron-hole excitations. Phase space factors strongly weight the large- $p$ portions of the continua, leading to peaks near the upper ends of the ranges. Note that there is no sharp onset of the electron-hole excitations, but the sharp high- $p$ cutoff at the bulk value remains as shown in fig. 4(a).

\subsection{Fluorescence near a single surface}

We consider here the specific problem of molecular fluorescence in the presence of a metal surface. The quantity of interest now is the decay rate $\gamma$ of an excited electronic state. According to the semiclassical theory of radiation this quantum mechanical decay rate is related to the classical dissipated power by

$$
\gamma=\mathscr{P} / \hbar \omega
$$

Thus, in the absence of nonradiative decay channels and when the molecule is far from the metal, the decay is purely radiative and is given by (3.25) in which $\mathscr{P}$ is given by the Larmor formula (3.11), 


$$
\gamma_{\mathrm{rad}}=|\boldsymbol{\mu}|^{2} \varepsilon_{1}^{1 / 2} \omega^{3} / 3 \hbar c^{3}
$$

Here $\boldsymbol{\mu}$ must be taken to be twice the quantum mechanical transition dipole moment for the radiating transition [1]. In this section, we use the principle embodied in (3.25) to discuss the various contributions to the decay rate.

In addition to radiative decay most fluorescing molecules possess nonradiative decay channels associated with the medium in which they are imbedded or with their internal structure. The total decay rate far from the metal is then the sum of the radiative decay rate $\gamma_{\mathrm{rad}}$ and a nonradiative decay rate $\gamma_{\mathrm{nr}}$. The radiative quantum efficiency is defined to be the ratio of the radiative decay rate to the total decay rate,

$$
\eta=\gamma_{\mathrm{rad}} /\left(\gamma_{\mathrm{rad}}+\gamma_{\mathrm{nr}}\right)
$$

In general one assumes that $\gamma_{n r}$ is independent of the molecule-metal spacing $d$. The picture here is that the nonradiative decay channel corresponds to an independent nonelectromagnetic decay that is unaffected by the presence of the metal. One would, for example, not expect this assumption to be correct for an adsorbed molecule.

When the molecule is spaced a distance $d$ from the metal surface, the total decay rate will be the sum of the nonradiative decay rate $\gamma_{\mathrm{nr}}$ and a rate given by the prescription (3.25) where $\mathscr{P}$ is the classical power dissipated given by (3.15),

$$
\gamma(d)=\gamma_{\mathrm{nr}}+\mathscr{P}(d) / \hbar \omega
$$

It is customary to introduce the reduced decay rate $\hat{\gamma}(d)$, the decay rate normalized with respect to its value far from the metal;

$$
\hat{\gamma}(d)=\gamma(d) / \gamma(d \rightarrow \infty) .
$$

We form this quantity using (3.28) with $\mathscr{P}(d)$ given by (3.15). Noting that $\gamma_{\mathrm{rad}}=\mathscr{P}(d \rightarrow \infty) / \hbar \omega$, with $\mathscr{P}(d \rightarrow \infty)$ given by (3.11), and using (3.27), we can write

$$
\begin{aligned}
\hat{\gamma}(d)= & 1-\eta+\frac{3}{2} \eta k_{1}^{-3} \operatorname{Re} \int_{0}^{\infty} \mathrm{d} p \frac{p}{q_{1}}\left\{\frac{\mu_{\perp}^{2} p^{2}}{|\mu|^{2}}\left[1+r_{12}^{\mathrm{P}} \exp \left(2 \mathrm{i} q_{1} d\right)\right]\right. \\
& \left.+\frac{\mu_{\|}^{2} k_{1}^{2}}{2|\mu|^{2}}\left[1+r_{12}^{\mathrm{s}} \exp \left(2 \mathrm{i} q_{1} d\right)\right]+\frac{\mu_{\|}^{2} q_{1}^{2}}{2|\mu|^{2}}\left[1-r_{12}^{\mathrm{P}} \exp \left(2 \mathrm{i} q_{1} d\right)\right]\right\} .
\end{aligned}
$$

Here the second term exactly cancels the contribution to the integral arising from the terms not involving the reflection coefficients, thus we arrive at the alternative and somewhat simpler expression:

$$
\hat{\gamma}(d)=1+\frac{3}{2} \eta k_{1}^{-3} \operatorname{Re} \int_{0}^{\infty} \mathrm{d} p \frac{p \exp \left(2 \mathrm{i} q_{1} d\right)}{q_{1}|\mu|^{2}}\left\{\mu_{\perp}^{2} p^{2} r_{12}^{\mathrm{P}}+\frac{1}{2} \mu_{\|}^{2} k_{1}^{2} r_{12}^{\mathrm{s}}-\frac{1}{2} \mu_{\|}^{2} q_{1}^{2} r_{12}^{\mathrm{P}}\right\}
$$

This form of the result corresponds to eqs. (2.17) and (2.29) of Chance et al. [23]. 


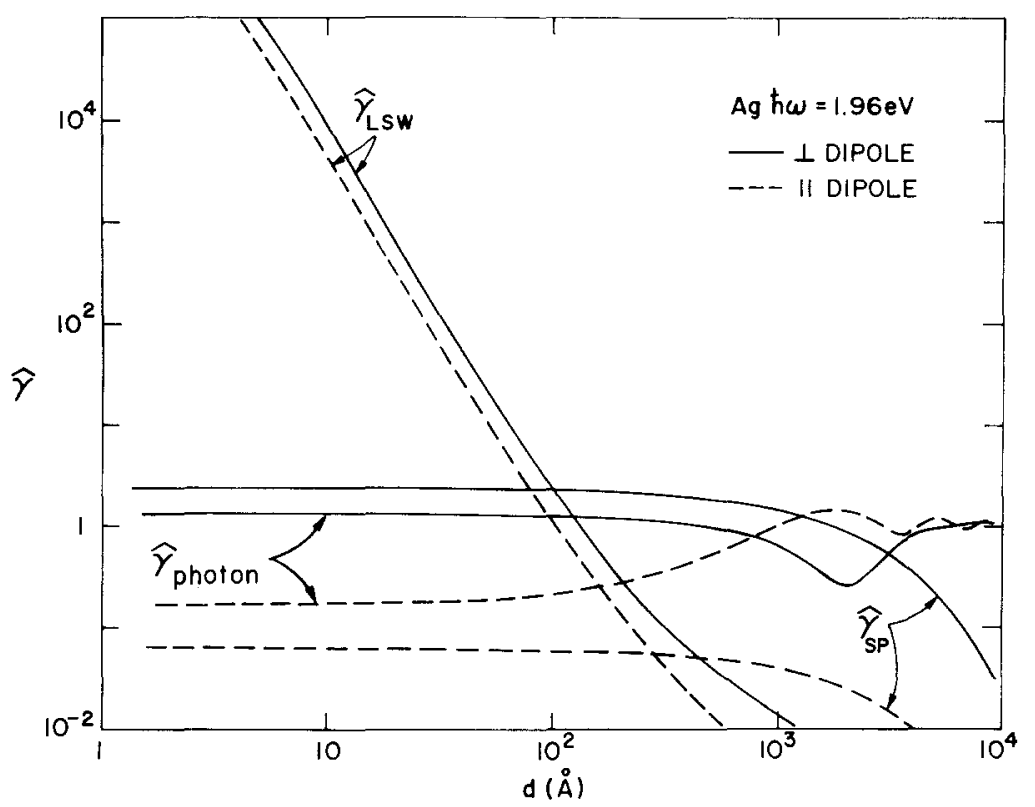

Fig. 6. Partial decay rates vs. distance for a dipole in vacuum outside a Ag surface calculated using the nonlocal SClB model, eqs. (2.20), (2.30), and (3.30) with $\eta=1$ and $\hbar \omega=1.96 \mathrm{eV}$. The Ag parameters are the same as those for fig. 4 .

The total normalized decay rate can be written as a sum of partial decay rates in a manner corresponding to the partitioning (3.17) of the dissipated power. For this purpose, we refer to the expression (3.30) and write

$$
\hat{\gamma}(d)=\hat{\gamma}_{\mathrm{nr}}+\hat{\gamma}_{\mathrm{photon}}(d)+\hat{\gamma}_{\mathrm{SP}}(d)+\hat{\gamma}_{\mathrm{LSW}}(d)
$$

where $\hat{\gamma}_{\mathrm{nr}}=1-\eta$ is the nonradiative reduced decay rate; $\hat{\gamma}_{\text {photon }}(d)$ is the normalized decay rate via photon emission and corresponds to the range $0<p<k_{1}$ in the integral of $(3.30) ; \hat{\gamma}_{\mathrm{SP}}$ is the normalized decay rate via surface plasmon emission and corresponds to the contribution of the pole in $r_{12}^{\mathrm{P}}$; and $\hat{\gamma}_{\mathrm{Lsw}}(d)$ is the normalized decay rate via lossy surface waves and corresponds to the high- $p$ continuum contribution to the integral. These various contributions to the decay were discussed in some detail in the previous section.

The partial decay rates discussed above are plotted in fig. 6 as a function of the molecule-metal distance. The solid curves are for a perpendicular, the dashed for a parallel orientation of the transition dipole moment. An alternative way of displaying these results is shown in fig. 7 , where we plot the relative decay probability vs. $d$ using the same parameters. For large $d$ the radiative decay rates dominate, and these have an oscillatory behavior due to interference. The surface plasmon decay is most important at intermediate distances $\left(10^{2}-10^{3} \AA\right)$. The lossy surface wave contribution always dominates at small $d$.

The results of figs. 6 and 7 are calculated with the reflection coefficients given by the nonlocal SCIB model, corresponding to (2.20) and (2.26) with $\varepsilon_{\ell}$ and $\varepsilon_{\mathrm{t}}$ given by the Lindhard form (2.30). The parameters are those appropriate for $\mathrm{Ag}$. The lossy surface wave decay is thus due mainly to electron-hole excitations, with a small electron scattering contribution. The importance of the nonlocal 


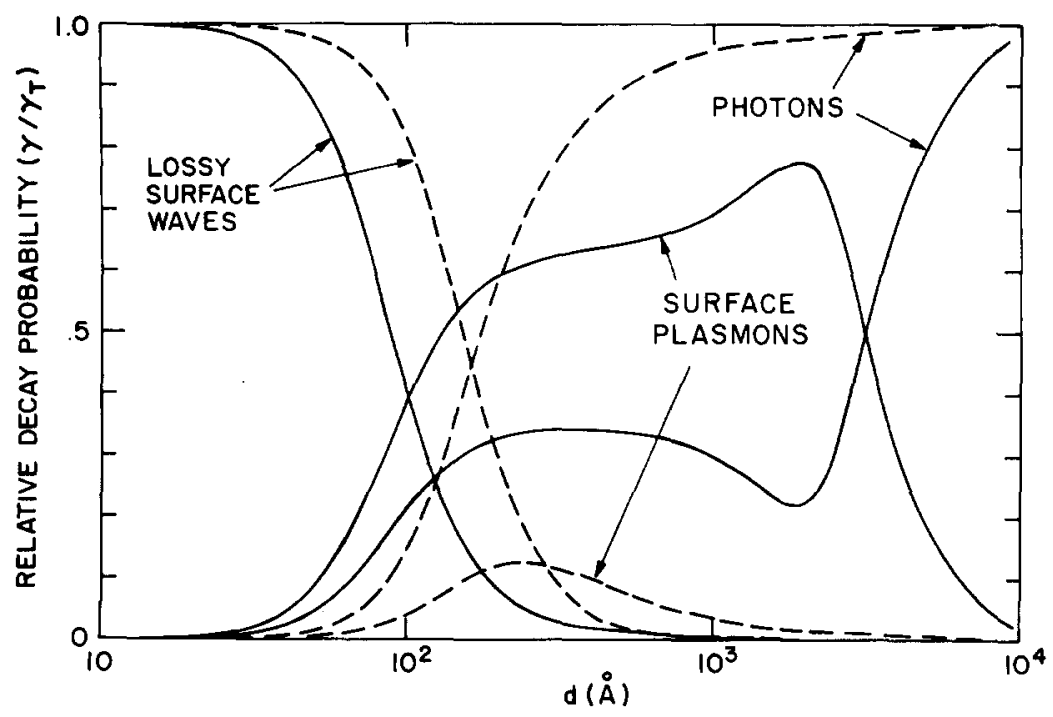

Fig. 7. Relative decay probabilities for the same parameters as used in the plots in fig. 6.

response at small $d$ is shown in fig. 8 , where we plot the ratio of the calculated decay rate to $\gamma_{\text {local }}$, the rate determined using the local result (3.23):

$$
\gamma_{\text {local }}\left(d \ll k_{1}^{-1}\right)=\left(8 \varepsilon_{1} \hbar d^{3}\right)^{-1} \operatorname{Im}\left[\left(\varepsilon_{2}-\varepsilon_{1}\right) /\left(\varepsilon_{2}+\varepsilon_{1}\right)\right]\left(\mu_{\perp}^{2}+\frac{1}{2} \mu_{\|}^{2}\right) \text {. }
$$

We have also separated out the electron scattering contribution, which is that part of $\gamma_{\mathrm{LSw}}$ proportional to $\nu$. We see in fig. 8 that due to the electron-hole excitations $\gamma_{L S w}$ increases more rapidly than $d^{-3}$, reaching a peak at $d \cong 3 \AA$, while the electron scattering contribution is gradually screened out.

It is of interest to compare results obtained from the SCIB model as shown in fig. 8 with results from the QIB model discussed in section 2.3. To be consistent with the quantum mechanical formulation

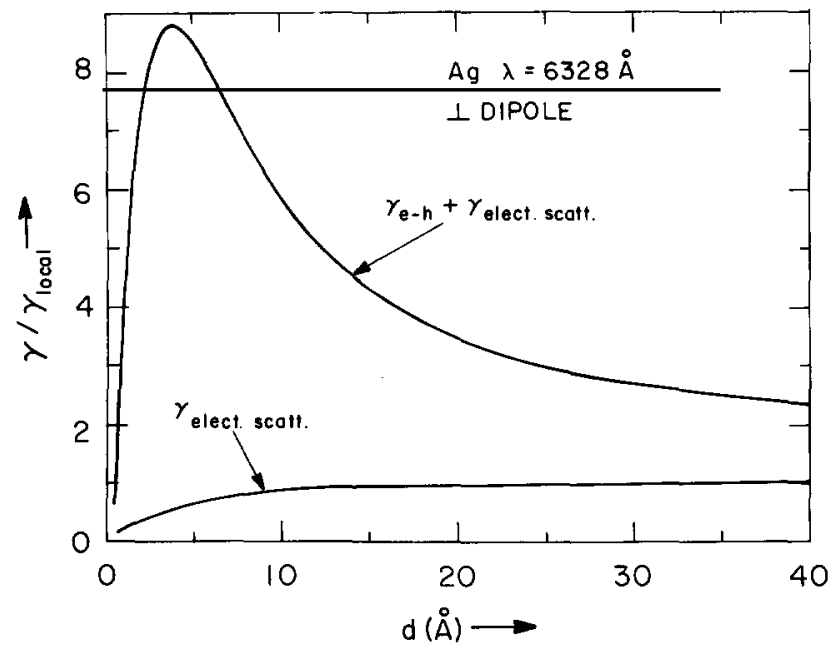

Fig. 8. Decay rate for a $\perp$ dipole outside a Ag surface calculated with the SCIB model and normalized with respect to the local result eq. (3.33). The lower curve is that part of the decay rate proportional to the electron scattering rate $\nu$. 
required in the QIB model we choose a hypothetical collisionless free-electron metal with no core polarization $\left(\varepsilon_{\mathrm{b}}=1\right)$ and an electron density corresponding to $\mathrm{Cu}$. The only loss mechanism is thus electron-hole excitation. For a perpendicular dipole and using the quasistatic approximation $\left(r_{12}^{\mathrm{S}}=0\right.$, $q_{1}=\mathrm{i} p$ ), the normalized decay rate (3.31) becomes:

$$
\hat{\gamma}(d)=1+\frac{3}{2} \eta k_{1}^{-3} \int_{0}^{\infty} \mathrm{d} p p^{2} \mathrm{e}^{-2 p d} \operatorname{Im}\left(r_{12}^{\mathrm{P}}\right)
$$

Figure 9 shows results for this formula using the two models. As expected from the results in fig. 3(b), $\hat{\gamma}_{\text {QIB }}$ is always less than $\hat{\gamma}_{\text {SCIB }}$. However, if the metal "surface" is chosen to be at the jellium edge for the QIB model, the two models agree much better. This is shown in fig. 9 where the dotted curve is the solid curve shifted to the right by $\sim 0.9 \AA$.

The conclusion to be made from this comparison is that the two models give qualitatively similar results, and with a slight shift in the effective molecule-metal spacing the results are quantitatively close as well. Which model to chose is to some extent a matter of taste. The QIB model certainly has a more realistic electron density profile while the SCIB model allows the inclusion, albeit in a phenomenological manner, of important effects such as core polarization and electron scattering. Furthermore the SCIB model is computationally much simpler; QIB calculations require at least a factor of 10 more computer time.

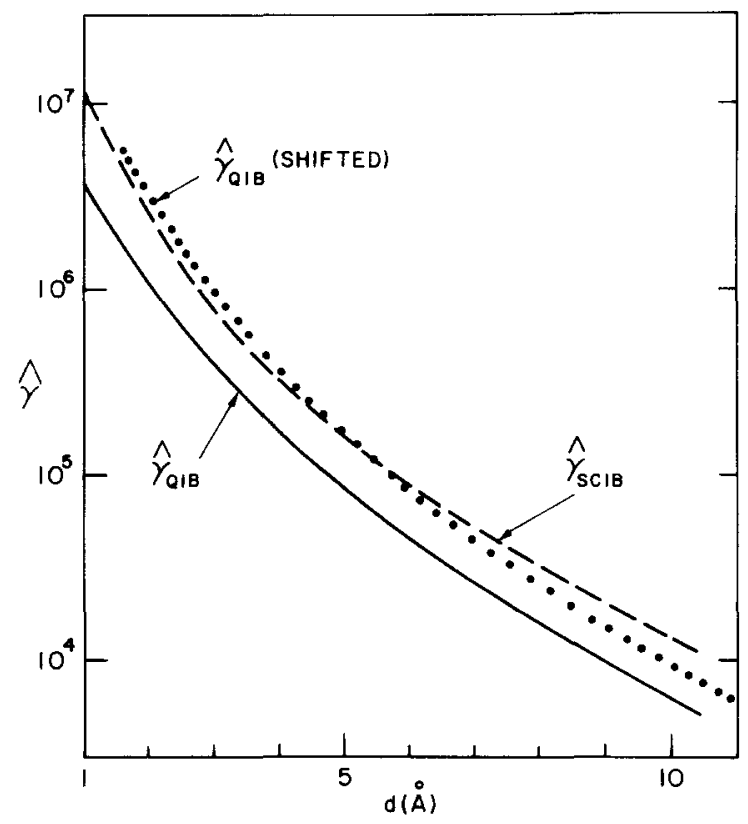

Fig. 9. Comparison between the decay rates for a $\perp$ dipole outside a free-electron metal with $\hbar \omega_{\mathrm{p}}=10.8 \mathrm{eV}$ obtained from the QIB (solid) and SCIB (dashed) models. The distance scale is measured from the infinite barrier in each case. The dotted curve is the QIB result shifted to the right by $0.9 \mathrm{~A}$, which corresponds to placing the effective surface at the jellium edge. 


\subsection{Dielectric layer above a metal}

We next consider the problem of a dielectric layer separating the radiating dipole from the metal, as shown in the inset of fig. 10. This geometry is important since it is often used in experiments, notably those by Drexhage [24, 25], by Campion et al. [38], and by Adams et al. [36]. It should be clear that the nonlocal response of the metal will have little effect. The point being that the dipole is spaced far from the metal so that the $p$-space power spectrum is cut off at large $p$ by the geometrical factor $\exp (-2 p t)$. This means that we can use the Fresnel formulas (2.6) and (2.7) for the reflection coefficients. The formula (3.30) for the normalized decay rate outside a metal surface can easily be generalized to obtain a formula appropriate to the geometry of fig. 10. To do this we simply replace the two-layer reflection coefficients $r_{12}^{\mathrm{P}}$ and $r_{12}^{\mathrm{S}}$ with their three-layer analogs [5]: $\dagger$

$$
r_{12}^{\mathrm{P}, \mathrm{S}} \rightarrow r_{123}^{\mathrm{P}, \mathrm{S}}=\left[r_{12}^{\mathrm{P}, \mathrm{S}}+r_{23}^{\mathrm{P}, \mathrm{S}} \exp \left(2 \mathrm{i} q_{2} t\right)\right] /\left[1+r_{12}^{\mathrm{P}, \mathrm{s}} r_{23}^{\mathrm{P}, \mathrm{S}} \exp \left(2 \mathrm{i} q_{2} t\right)\right]
$$

where $t$ is the layer thickness. In addition to the surface plasmon mode associated with the pole in $r_{23}^{P}$ (which is now shifted by the dielectric) we also have the possibility of waveguide modes associated with the vanishing of the denominators in $r_{123}^{\mathrm{P}}$ or $r_{123}^{\mathrm{s}}$.

As an example we show in fig. 10 the calculated power dissipated spectrum for a perpendicular dipole just outside a dielectric layer on a $\mathrm{Ag}$ film. We have taken $d=2 \AA$, but the results are insensitive to $d$ as long as $d \ll t, \lambda$. For the value $t=8000 \AA$ there are two waveguide modes $\mathrm{TM}_{0}$ and $\mathrm{TM}_{1}$ in addition to the surface plasmon, and they all produce prominent peaks in $d \mathscr{P} / \mathrm{d} p$. For a parallel dipole the power dissipated spectrum would have peaks at the same places as those in fig. 10, as well as

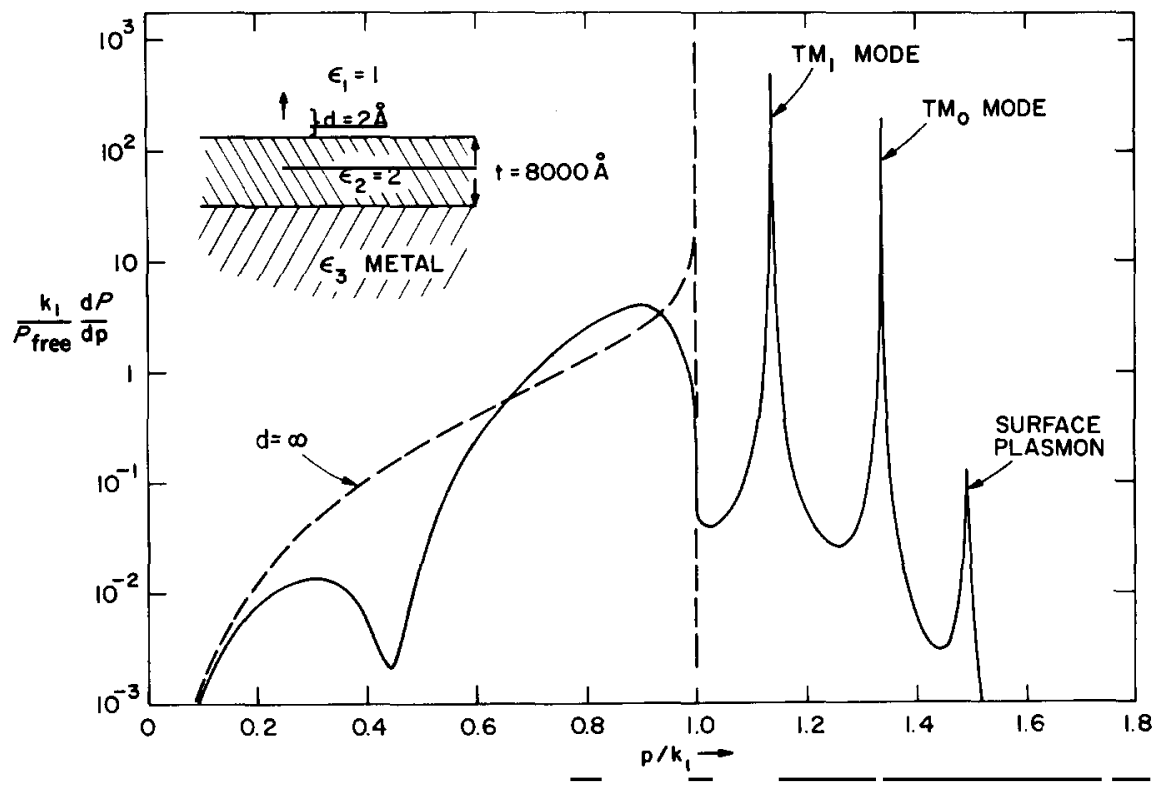

Fig. 10. Calculated $p$-space power spectrum at $\hbar \omega=1.96 \mathrm{eV}$ for a $\perp$ dipole $2 \AA$ above a dielectric on a $\mathrm{Ag}$ surface. The dashed curve is the form expected when the dipole is in free space $(d=\infty)$.

$\dagger$ This result, which is derived in ref. [5] pp, 464-472, can be obtained by summing plane wave amplitudes as outlined in the following section. 
additional peaks corresponding to the TE waveguide modes. For comparison we also show in the dashed curve of fig. 10 the result for a dipole in free space $(d=\infty)$. The structure in the solid curve in the radiation region below the light line arises from interference.

The formal calculation of the decay rate into each of the modes in fig. 10 can be done by expanding eq. (3.35) in the vicinity of each pole and evaluating the pole contribution as was done in eqs. (3.20) and (3.21). For computational purposes, however, a direct numerical integration of the pole contributions is simpler.

In fig. 11 we show results of a numerical integration to obtain the decay rates for the same structure as in fig. 10. The $\mathrm{TM}_{0}$ and $\mathrm{TM}_{1}$ modes have cutoffs at $t=2712$ and $5875 \AA$, respectively. The normalized decay rate into each mode is zero at cutoff, increases rapidly above cutoff to a maximum, and then falls slowly thereafter. This result can be understood by realizing that the decay rate is a measure of the overlap between the near fields of the dipole and the fields of the mode. At cutoff the mode field extends to $z=+\infty$, consequently there is a poor match with the dipolar fields. As the thickness of the dielectric is increased above cutoff, the mode field is more localized, providing a better match with the dipolar fields. As the thickness of the dielectric is further increased, however, the mode spreads approximately as the guide thickness, thus producing a poorer match with the dipolar fields.

The surface plasmon decay rate $\hat{\gamma}_{\mathrm{SP}}$ actually peaks when $t \cong 600 \AA$, a somewhat surprising result, since eq. (3.21) shows an exponential falloff with distance from the metal. The peak in $\hat{\gamma}_{\mathrm{SP}}$ with finite $t$ comes about because in the range $t \cong \lambda / 10$ the SP field is concentrated in the dielectric and thereby provides a better match to the dipolar fields. At large values of $t$, the SP field is again concentrated on the metal and the exponential falloff is recovered.

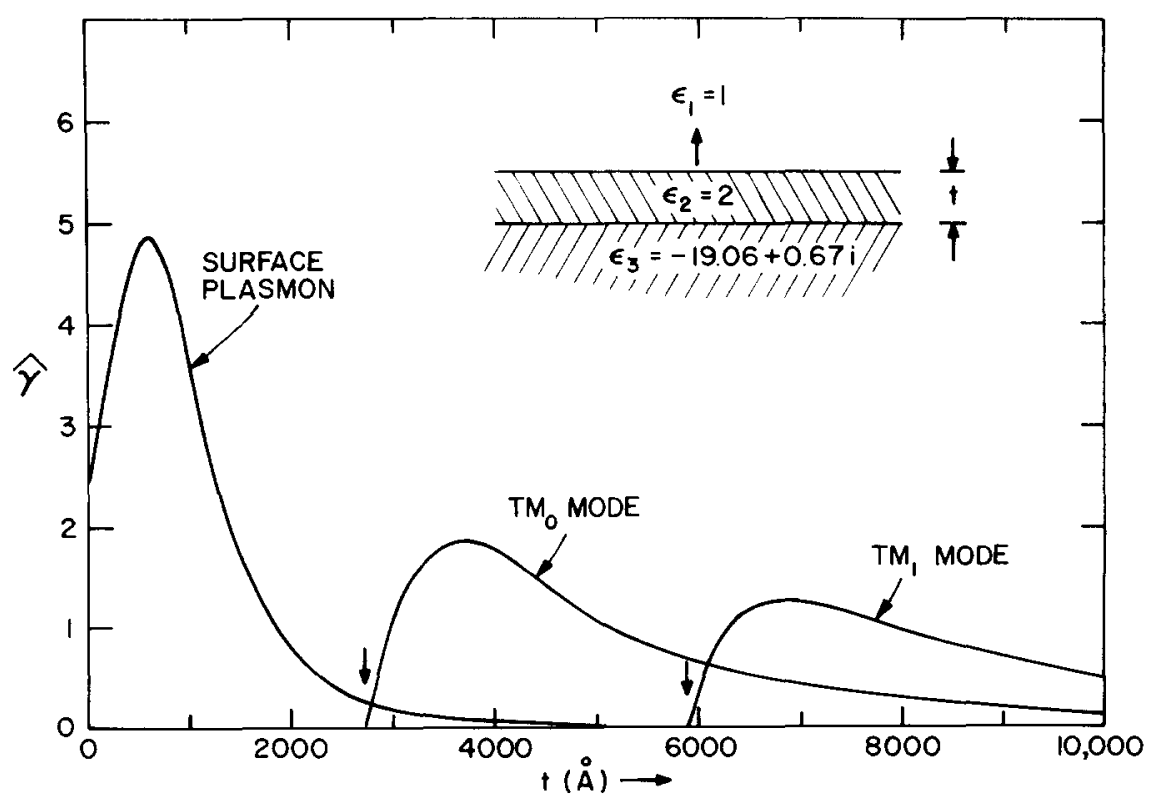

Fig. 11. Normalized decay rates into the various guided modes as a function of the dielectric spacer thickness for a $\perp$ dipole $2 \AA$ above the dielectric. The parameters correspond to $\mathrm{Ag}$ at $\hbar \omega=1.96 \mathrm{eV}$. The vertical arrows indicate the cutoffs for the first two waveguide modes. 


\subsection{Tunnel junction geometry}

We now address the problem of a radiating dipole in a thin oxide layer sandwiched between two metal films as shown in fig. 12. This is the geometry used in the Raman scattering experiments by Tsang et al. [47] and in the experiments on light emission via inelastic tunneling (LEIT) by Lambe and McCarthy [48]. Although our calculations are done specifically for a point dipole in the oxide of the junction, which is appropriate for the fluorescence or Raman scattering problem, to the extent that the fluctuating tunneling current can be confined to the oxide, the results are also applicable to the LEIT devices $[49,50]$.

The usual procedure for treating the problem shown in fig. 12 (see, e.g., Chance et al. [23]) is to form for each value of $p$ the appropriate downward and upward propagating solutions to Maxwell's equations with unknown coefficients in each of the five regions. There will be six coefficients for regions $2-4$ and one each for regions 1 and 5. Matching boundary conditions at each interface then produces eight equations in eight unknowns, which can be solved to find the field at the dipole. This procedure requires considerable algebra. We give here a simpler derivation which uses our general method of describing the surface response by reflection coefficients. This has the further advantage of giving an intuitive picture of the physical quantities involved in the calculation.

In the expression (3.30) for the normalized decay rate for a dipole above a single surface, the three factors $r_{12}^{\mathrm{P}} \exp \left(2 \mathrm{i} q_{1} d\right), r_{12}^{\mathrm{S}} \exp \left(2 \mathrm{i} q_{1} d\right)$, and $-r_{12}^{\mathrm{P}} \exp \left(2 \mathrm{i} q_{1} d\right)$, give the complex amplitude changes, respectively, for the $\perp E$-field of a P-polarized wave, the $\| E$-field of an S-polarized wave, and the $\| E$-field of a P-polarized wave, produced as each wave propagates from $z=d$ to the metal and back. When more boundaries are added to the problem we need only generalize these three quantities, allowing for all multiple reflections, to obtain the resulting field at the dipole.

We consider first the $\perp E$-field from the P-polarized waves for the geometry in fig. 12 . Define $a=r_{345}^{\mathrm{P}} \exp \left(-2 \mathrm{i} q_{3} z_{3}\right)$ and $b=r_{321}^{\mathrm{P}} \exp \left(2 \mathrm{i} q_{3} z_{2}\right)$, the amplitude changes produced when a wave propagates from the plane of the dipole $(z=0)$ to the metal below and back and to the metal above and back, respectively. In our discussion the interest will be in the low- $p$ regime, in which case the metal response can be described by a local dielectric constant and the three-layer reflection coefficients will be given by

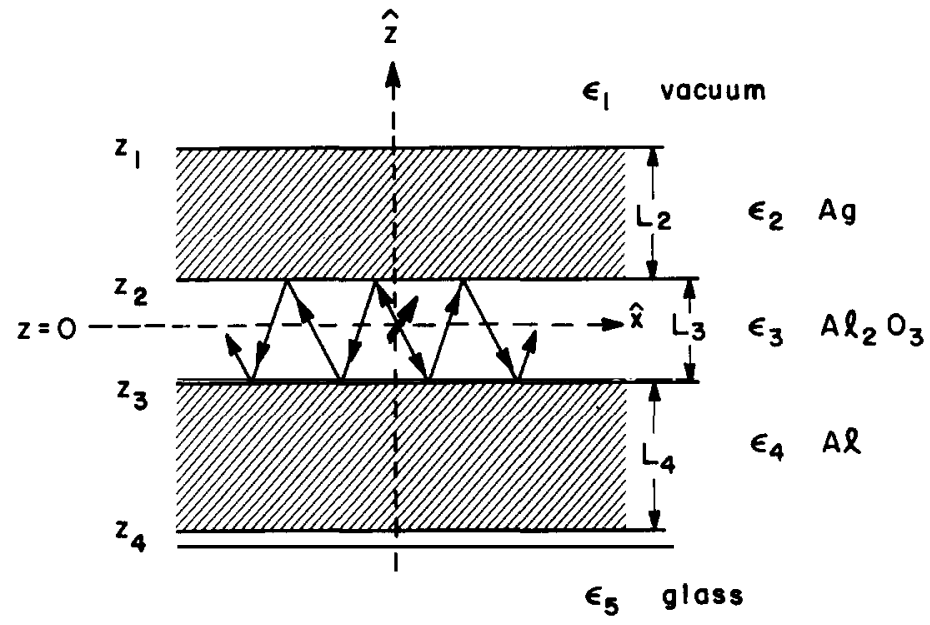

Fig. 12. Diagram for summing the plane-wave amplitudes contributing to the decay rate of a dipole inside a dielectric sandwiched between two metal films. 
(3.35) with the appropriate changes in subscripts,

$$
r_{i j k}^{\mathrm{P}, \mathrm{S}}=\left[r_{i j}^{\mathrm{P}, \mathrm{s}}+r_{j k}^{\mathrm{P}, \mathrm{s}} \exp \left(2 \mathrm{i} q_{j} L_{j}\right)\right] /\left[1+r_{i j}^{\mathrm{P}, \mathrm{s}} r_{j k}^{\mathrm{P}, \mathrm{s}} \exp \left(2 \mathrm{i} q_{j} L_{j}\right)\right] .
$$

The multiple reflections which must be summed are represented pictorially by the zig-zag arrows in fig. 12; each crossing of the plane $z=0$ contributes one term to the sum. The sum associated with the arrows going to the right is

$$
a+a b+a^{2} b+a^{2} b^{2}+a^{3} b^{2}+a^{3} b^{3}+\cdots
$$

and the sum associated with the arrows to the left is

$$
b+a b+a b^{2}+a^{2} b^{2}+a^{2} b^{3}+a^{3} b^{3}+\cdots .
$$

Summing these geometric series we obtain

$$
A_{1}^{\mathrm{P}}=(1+a)(1+b) /(1-a b)-1 \text {, }
$$

where $A_{\perp}^{\mathrm{P}}$ is defined as the amplitude change in the $\perp E$-field component of the P-polarized wave produced by all reflections from the metal surfaces. For the $\|$ component of the S-polarized waves, the sums are exactly the same as above. Thus $A_{\|}^{\mathrm{S}}$ is given by (3.37) with $a=r_{345}^{\mathrm{s}} \exp \left(-2 \mathrm{i} q_{3} z_{3}\right)$ and $b=r_{321}^{\mathrm{S}} \exp \left(2 \mathrm{i} q_{3} z_{2}\right)$. For the $\|$ component of the P-polarized waves, there is a sign change at each reflection, thus $A_{\|}^{\mathrm{P}}$ is given by (3.37) with $a=-r_{345}^{\mathrm{P}} \exp \left(-2 \mathrm{i} q_{3} z_{3}\right)$ and $b=-r_{321}^{\mathrm{P}} \exp \left(2 \mathrm{i} q_{3} z_{2}\right)$. Collecting these results together and modifying eq. (3.30) for the dipole being in a medium with dielectric constant $\varepsilon_{3}$, we find for the normalized decay rate

$$
\begin{aligned}
\hat{\gamma}= & 1-\eta+\frac{3}{2} \eta k_{1}^{-3}|\boldsymbol{\mu}|^{-2} \operatorname{Re} \int_{0}^{\infty} \mathrm{d} p \frac{p}{q_{3}}\left\{\mu^{2} p^{2}\left[\frac{\left[1+r_{345}^{\mathrm{P}} \exp \left(-2 \mathrm{i} q_{3} z_{3}\right)\right]\left[1+r_{321}^{\mathrm{P}} \exp \left(2 \mathrm{i} q_{3} z_{2}\right)\right]}{\mathrm{P}}\right]\right. \\
& +\frac{1}{2} \mu_{\|}^{2} k_{3}^{2}\left[\frac{\left[1+r_{345}^{\mathrm{s}} \exp \left(-2 \mathrm{i} q_{3} z_{3}\right)\right]\left[1+r_{321}^{\mathrm{s}} \exp \left(2 \mathrm{i} q_{3} L_{3}\right)\right.}{1-r_{321}^{\mathrm{s}} r_{345}^{\mathrm{s}} \exp \left(2 \mathrm{i} q_{3} L_{3}\right)}\right] \\
& \left.+\frac{1}{2} \mu_{\|}^{2} q_{3}^{2}\left[\frac{\left[1-r_{345}^{\mathrm{P}} \exp \left(-2 \mathrm{i} q_{3} z_{3}\right)\right]\left[1-r_{321}^{\mathrm{P}} \exp \left(2 \mathrm{i} q_{3} z_{2}\right)\right]}{1-r_{321}^{\mathrm{P}} r_{345}^{\mathrm{P}} \exp \left(2 \mathrm{i} q_{3} L_{3}\right)}\right]\right\}
\end{aligned}
$$

As an example we show in fig. 13 a portion of the normalized $p$-space power spectrum, identified as the integrand in (3.38) multiplied by the factors preceding the integral. In the calculation we have taken $\eta=1$ and specialized to the $\perp$ orientation of the dipole. The dipole is in the oxide layer and the lower electrode was taken to be infinitely thick, which is a reasonable approximation to the actual devices.

There are two guided modes of interest now as indicated by the two peaks in the power spectrum in fig. 13. The larger peak arises from the vanishing of the quantity $1-r_{321}^{\mathrm{P}} r_{345}^{\mathrm{P}} \exp \left(2 \mathrm{i} q_{3} L_{3}\right)$ in the denominator of $\mathrm{d} \mathscr{P} / \mathrm{d} p$. This pole corresponds to the slow wave or junction mode occurring at $p_{\mathrm{JM}} \cong 10 k_{1}$ in fig. 13 . The fields associated with this mode are peaked in the oxide and decay rapidly outside; consequently the dipole couples very strongly to it. The smaller peak arises from a pole in the 


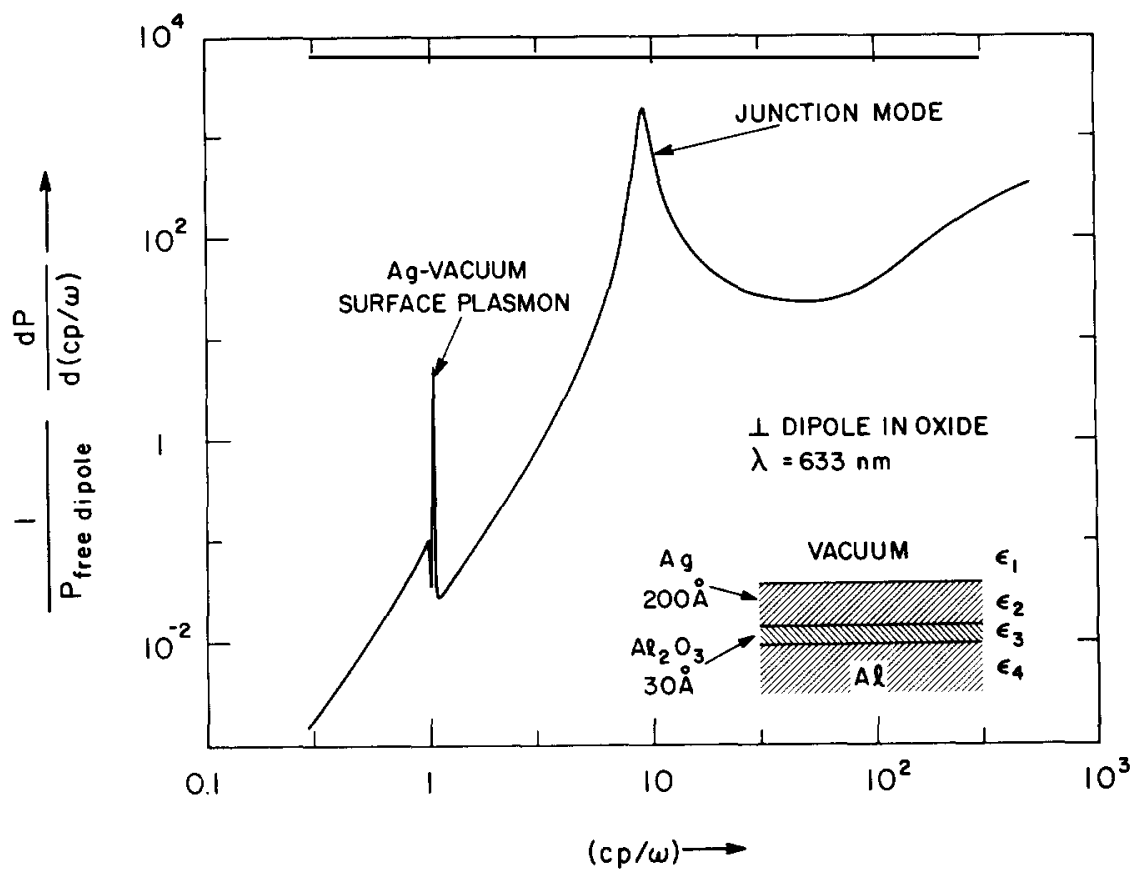

Fig. 13. Calculated $p$-space power spectrum for a $\perp$ dipole in the oxide of a tunnel junction as shown in the inset. The parameters are $\eta=1$, $\hbar \omega=1.96 \mathrm{eV}, \varepsilon_{1}=1, \varepsilon_{2}=-19+0.5 \mathrm{i}, \varepsilon_{3}=3.1, \varepsilon_{4}=-50+17 \mathrm{i}$.

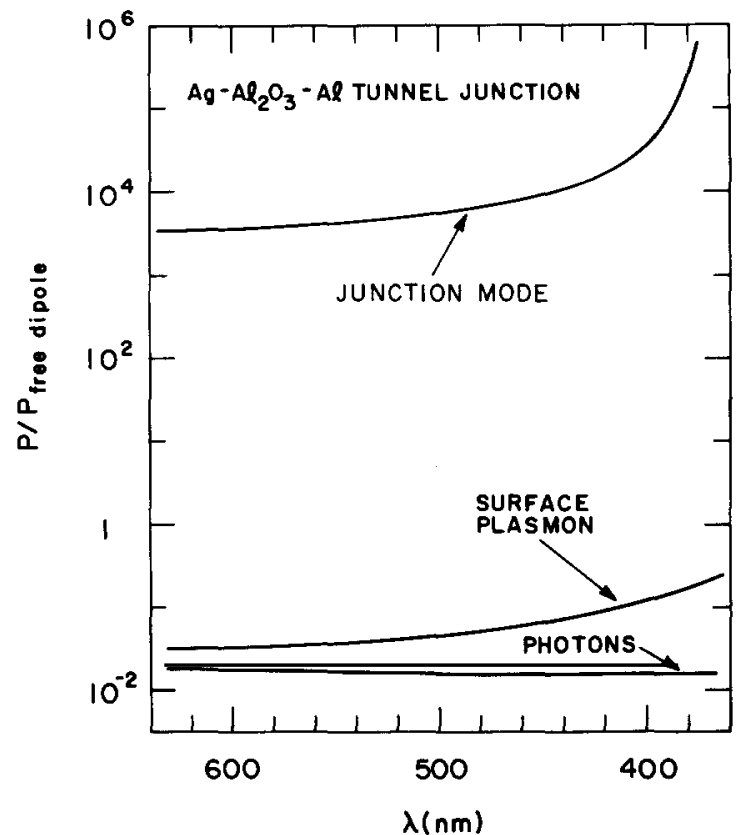

Fig. 14. Normalized power dissipation vs. frequency (equivalent to the normalized decay rate) for a $\perp$ dipole in the oxide of the tunnel junction shown in the inset of fig. 13 . 
two-layer reflection coefficient $r_{21}^{\mathrm{P}}$ occurring in $r_{321}^{\mathrm{P}}$. This pole corresponds to the surface plasmon mode localized on the Ag-vacuum interface. It is modified slightly by the multilayered structure. The fields of the surface plasmon are peaked at the vacuum interface and extend well into the vacuum; consequently the coupling of the dipole field to it is much weaker than to the junction mode. This feature is demonstrated in fig. 14 where we plot as a function of frequency the powers emitted into the two guided modes as well as into free photons. These are all normalized with respect to the power emitted by a dipole in an infinite medium $\varepsilon_{1}$, which has the effect of suppressing the $\omega^{4}$ dependence. The most striking feature in fig. 14 is the complete dominance of the junction mode.

\subsection{Fluorescence near a small sphere}

We conclude this chapter with a discussion of a fluorescing molecule near a small sphere whose response is described by a local dielectric constant. This discussion is pertinent to a large class of experiments on fluorescence and Raman scattering from molecules on metal-island films [51-53], on particle arrays $[54,55]$, and in metal-colloid solutions [56-59]. Theoretical treatments of this problem have been given by several authors [60-62].

Consider a point dipole oscillating harmonically at frequency $\omega$ and placed near a sphere in a medium with dielectric constant $\varepsilon_{1}$ as shown in fig. 15. We are interested in the case of a molecule placed close to a sphere whose radius is small compared with the wavelength of the emitted radiation $(a, R \ll c / \omega)$. It is therefore appropriate to use the quasistatic approximation. On the other hand, if the sphere is metal, it should not be so small that nonlocal effects are important. This means we must assume $k_{\mathrm{F}} a \gg 1$. Our aim here will be to calculate the power dissipated by the dipole, and as in the planar case discussed in section 3.1, we must calculate the "reflected" or "induced" field at the dipole due to the presence of the sphere. In the planar case the effect of the metal surface was described by the reflection coefficients; in the spherical case the role of the reflection coefficients is taken by the multipole polarizabilities, which are introduced below.

The quasistatic potential due to the dipole is

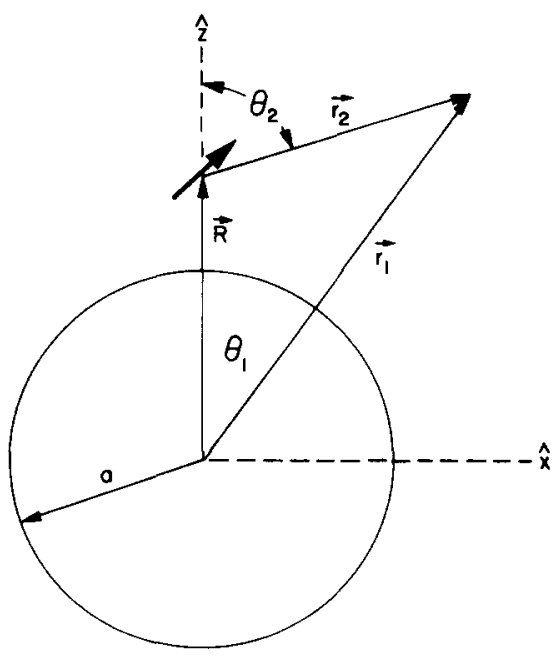

Fig. 15. Geometry for calculating the decay rate for a dipole near a sphere. 


$$
\Phi_{\text {dipole }}=\left(\mu \cdot r_{2}\right) / \varepsilon_{1} r_{2}^{3}=\left(\mu_{\perp} / \varepsilon_{1} r_{2}^{2}\right) P_{1}\left(\cos \theta_{2}\right)+\left(\mu_{\|} / \varepsilon_{1} r_{2}^{2}\right) P_{1}^{1}\left(\cos \theta_{2}\right) \cos \phi
$$

where $\mu_{\perp}$ and $\mu_{\|}$are the components of $\boldsymbol{\mu}$ perpendicular and parallel to the surface of the sphere beneath the dipole. Here $P_{l}^{m}$ is the associated Legendre polynomial and $\phi$ is the azimuthal angle. We now use the formula [63],

$$
\frac{1}{r_{2}^{2}} P_{1}^{m}\left(\cos \theta_{2}\right)=(-1)^{m+1} \sum_{l=m}^{\infty}\left(\begin{array}{l}
l+1 \\
l+m
\end{array}\right) \frac{r_{1}^{l}}{R^{l+2}} P_{l}^{m}\left(\cos \theta_{1}\right), \quad r_{1}<R,
$$

where $\left(\begin{array}{c}N \\ M\end{array}\right) \equiv N ! / M !(N-M) !$ is the binomial coefficient. Using this result the potential due to the dipole in the region $a<r<R$ can be written

$$
\Phi_{\text {dipole }}(\boldsymbol{r})=\frac{1}{\varepsilon_{1}} \sum_{m=0}^{1}\left(-\mu_{\perp} \delta_{m, 0}+\mu_{\|} \delta_{m, 1}\right) \sum_{l=m}^{\infty}\left(\begin{array}{l}
l+1 \\
l+m
\end{array}\right) \frac{r_{1}^{l}}{R^{l+2}} P_{l}^{m}\left(\cos \theta_{1}\right) \cos m \phi .
$$

The total potential just outside the sphere must be the sum of this field and the induced field from the sphere.

The induced field can be expressed in terms of the multipole polarizabilities, which characterize the response of the sphere to applied multipole fields $[42,64]$. For an applied $2 l$-pole field the potential just outside the sphere must be of the form

$$
\Phi_{l m} \propto\left(-r_{1}^{l}+\alpha_{l} / \varepsilon_{1} r_{1}^{l+1}\right) P_{l}^{m}\left(\cos \theta_{1}\right) \cos m \phi
$$

where the first term in parentheses corresponds to the potential of the applied field while the second corresponds to the potential of the induced field, due to the $2 l$-pole induced within the sphere. The coefficient $\alpha_{l}$ is the multipole polarizability.

The total potential, $\Phi=\Phi_{\text {dipole }}+\Phi_{\text {ind }}$, just outside the sphere must be a sum of potentials of the form (3.42). Therefore, from (3.41) we find

$$
\Phi_{\mathrm{ind}}(r)=\frac{1}{\varepsilon_{1}^{2}} \sum_{m=0}^{1}\left(\mu_{\perp} \delta_{m, 0}-\mu_{\|} \delta_{m, 1}\right) \sum_{l=m}^{\infty}\left(\begin{array}{c}
l+1 \\
l+m
\end{array}\right) \frac{\alpha_{l}}{R^{l+2} r_{1}^{l+1}} P_{l}^{m}\left(\cos \theta_{1}\right) \cos m \phi
$$

The induced electric field is given by $E_{\text {ind }}(r)=-\nabla \Phi_{\text {ind }}(r)$, and after some calculation the field at the dipole is found to be

$$
\boldsymbol{E}_{\text {ind }}(\boldsymbol{R})=\frac{1}{\varepsilon_{1}^{2}} \sum_{l=1}^{\infty} \frac{\alpha_{l}}{R^{2 l+4}}\left[(l+1)^{2} \mu_{\perp} \hat{z}+\frac{1}{2} l(l+1) \mu_{\|} \hat{\boldsymbol{x}}\right] .
$$

The power dissipated by the dipole is given by inserting (3.49) into (3.1), giving

$$
\mathscr{P}=\sum_{l=1}^{\infty} \frac{\omega}{2 \varepsilon_{1}^{2}}(l+1)\left[(l+1) \mu_{\perp}^{2}+\frac{1}{2} l \mu_{\|}^{2}\right] \operatorname{Im}\left(\alpha_{l}\right) / R^{2 l+4} .
$$

This formula is the analog of the plane surface result (3.22) in which the integral over the surface wavevector $p$ is replaced by a sum over $l$ and the quasistatic reflection coefficient is replaced by the 
multipole polarizability. Like its plane wave analog, (3.45) is quite general, it can be applied to any model for which the multipole polarizabilities can be calculated.

For our model of the sphere having a local dielectric constant $\varepsilon_{2}$, the $\alpha_{l}$ are given by

$$
\alpha_{i}=\varepsilon_{1} \frac{l\left(\varepsilon_{2}-\varepsilon_{1}\right)}{t \varepsilon_{2}+(t+1) \varepsilon_{1}} a^{2 l+1}
$$

Thus the dissipated power becomes

$$
\mathscr{P}=\frac{\omega}{2 \varepsilon_{1}} \sum_{l=1}^{\infty}(l+1)\left[(l+1) \mu_{\perp}^{2}+\frac{1}{2} l \mu_{\|}^{2}\right] \underline{\operatorname{Im}}\left\{\frac{l\left(\varepsilon_{2}-\varepsilon_{1}\right)}{l \varepsilon_{2}+(l+1) \varepsilon_{1}}\right\} \frac{a^{2 l+1}}{R^{2 l+4}} .
$$

When $a / R \ll 1$, the series in this expression is dominated by the dipole term $(l=1)$, and the reflected field is just that expected from a polarizable point at the center of the sphere. When $a / R \approx 1$, however, the power series is dominated by the large $l$ terms, and quite a different limit is reached. To see this limit we set $R=a+d$ and expand (3.47) for $d / a \ll 1$, giving

$$
\mathscr{P}=\frac{\omega}{2 \varepsilon_{1}} \sum_{l=1}^{\infty}(l+1)\left[(l+1) \mu_{\perp}^{2}+\frac{1}{2} l \mu_{i]}^{2}\right] \operatorname{Im}\left\{\frac{l\left(\varepsilon_{2}-\varepsilon_{1}\right)}{l \varepsilon_{2}+(l+1) \varepsilon_{1}}\right\} \frac{1}{a^{3}}(1+d / a)^{-(2 l+4)} .
$$

Now taking the limit $l \rightarrow \infty$, making the substitution $p=(l+1) / a$, and changing the sum to an integral we have

$$
\begin{aligned}
\mathscr{P} & \cong \frac{\omega}{2 \varepsilon_{1}} \int_{0}^{\infty} p^{2} \mathrm{~d} p\left[\mu_{\perp}^{2}+\frac{1}{2} \mu_{\|}^{2}\right] \operatorname{Im}\left\{\frac{\varepsilon_{2}-\varepsilon_{1}}{\varepsilon_{2}+\varepsilon_{1}}\right\} \mathrm{e}^{-2 p d} \\
& =\frac{\omega}{8 \varepsilon_{1} d^{3}}\left(\mu_{\perp}^{2}+\frac{1}{2} \mu_{\|}^{2}\right) \operatorname{Im}\left\{\frac{\varepsilon_{2}-\varepsilon_{1}}{\varepsilon_{2}+\varepsilon_{1}}\right\}
\end{aligned}
$$

This result is precisely the same as the corresponding plane surface result (3.23), which is to be expected. When the dipole is very close to the sphere, the reflected field at the dipole is the same as that from a semi-infinite planar surface.

The result (3.47) can be turned into a formula for the normalized decay rate for a molecule placed near a sphere. Using the prescription: $\hat{\gamma}(R)=1+\eta / \mathscr{P}_{\text {free }}$, where $\mathscr{P}_{\text {free }}$ is the power radiated by a free dipole given by the Larmor formula (3.11). The result is

$$
\hat{\gamma}(R)=1+\frac{3}{2} \eta\left(k_{1} R\right)^{-3} \mu^{-2} \sum_{l=1}^{\infty}(l+1)\left[(l+1) \mu_{\perp}^{2}+\frac{1}{2} l \mu_{\|}^{2}\right] \operatorname{Im}\left\{\frac{l\left(\varepsilon_{2}-\varepsilon_{1}\right)}{l \varepsilon_{2}+(l+1) \varepsilon_{1}}\right\}\left(\frac{a}{R}\right)^{2 l+1} .
$$

The result of a calculation using this formula for a $\perp$ dipole and a $\mathrm{Ag}$ sphere of radius $100 \AA$ is shown in the solid curve in fig. 16. The dashed curved labeled "point" is obtained by keeping only the $l=1$ term in (3.50), in which case the sphere response is that of a polarizable point. The dashed curve labeled "plane" is the plane-surface result obtained by replacing the sum in (3.50) by its large- $l$ limit corresponding to (3.49). Note the transition from "point" response far from the sphere to "plane" response close to the sphere, which occurs at $R \cong 2 a$. 


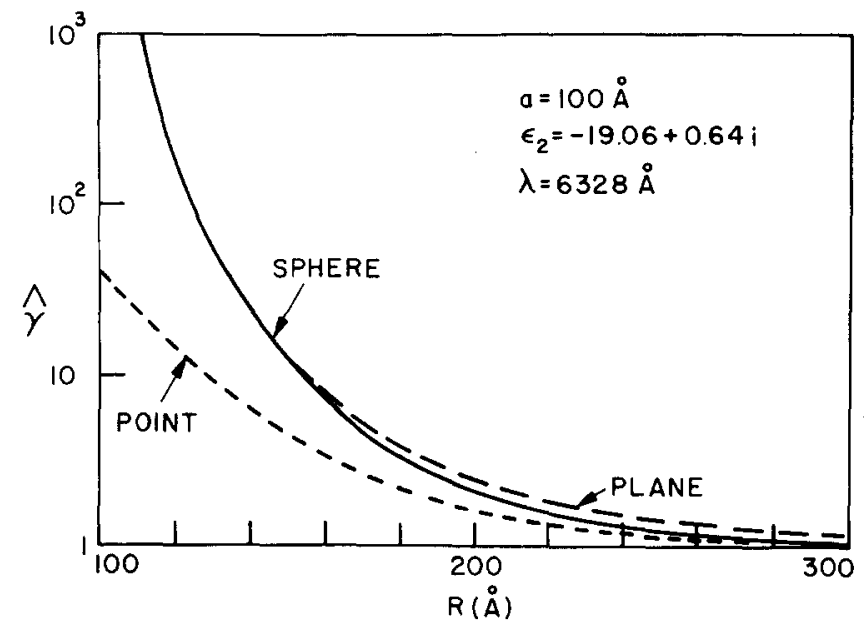

Fig. 16. Normalized decay rate for a dipole outside a sphere calculated with eq. (3.50). The dashed curves labeled "point" and "plane" correspond, respectively, to approximate solutions valid when the dipole is either very far from or very close to the sphere.

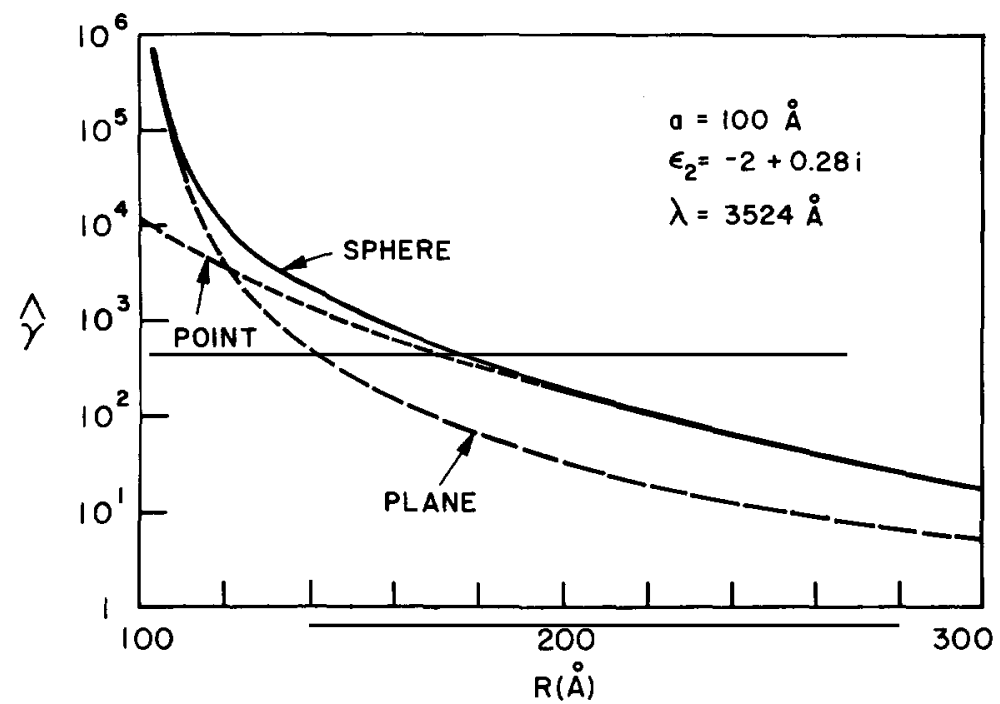

Fig. 17. Same calculation as in fig. 16, but for a frequency at which the dipole polarizability of the sphere has a resonance.

A similar plot is shown in fig. 17 , but there the frequency has been chosen so that $\operatorname{Re}\left\{\varepsilon_{2}\right\}=-2$, corresponding to a resonance in the dipole polarizability $\alpha_{1}$. In this case, as one might expect, the "point" response persists to much closer spacings.

We emphasize that in this section we have been considering the power dissipated by losses within the sphere. There is in addition power radiated by the dipole plus the sphere acting as a driven antenna. This radiation is discussed in section 5.4 .

\section{Shift and broadening of molecular vibration modes}

In chapter 3 we discussed the fluorescence problem, in which the molecule is generally well separated 
from the metal surface and there is little question as to the adequacy of electromagnetic methods. In this chapter we consider the influence of the surface on the vibrational modes of the molecule, which is taken to be very close to or even adsorbed onto it. There will therefore be important nonelectromagnetic effects. These we try to take into account by separate arguments, but we should make clear our view that one should not expect the sort of quantitative accuracy of prediction one has in the fluorescence applications. Rather, the aim will be to build intuition and in particular to investigate to what extent the physical picture of a dipole interacting with its image is meaningful.

The earliest discussions [65-67] of the effects we consider were based on the simple picture of a point-like molecule placed above a perfectly reflecting metal surface. The shift in a molecular vibrational frequency due to the presence of the metal surface can be calculated in this case by the following brief argument. We assume the molecule has a single mode vibrating perpendicular to the surface. The dipole polarizability of the free molecule, i.e., the molecule far from the surface, is of the phenomenological form

$$
\alpha(\omega)=\alpha_{\mathrm{e}}+\alpha_{\mathrm{v}} /\left[1-\left(\omega / \omega_{\mathrm{v}}\right)^{2}\right]
$$

where $\alpha_{\mathrm{e}}$ is the electronic (high-frequency) polarizability, $\alpha_{\mathrm{v}}$ is the polarizability associated with the vibrational mode, and $\omega_{\mathrm{v}}$ is the frequency of the mode. When the molecule is placed at height $d$ above the surface in the presence of an applied electric field $E_{0} \mathrm{e}^{-\mathrm{i} \omega t}$ perpendicular to the surface, the induced dipole moment will be $\mu(\omega) \mathrm{e}^{-\mathrm{i} \omega t}$, where

$$
\mu(\omega)=\alpha(\omega)\left[E_{0}+\mu(\omega) / 4 d^{3}\right]
$$

Here the quantity in square brackets is the amplitude of the total external electric field at the dipole, which is the sum of the applied field and the field due to the image dipole a distance $d$ below the surface. Solving for $\mu(\omega)$, we find

$$
\mu(\omega)=\frac{\alpha(\omega)}{1-\alpha(\omega) / 4 d^{3}} E_{0}
$$

The resonant frequency is the frequency at which $\mu(\omega)$ has a pole, i.e., using (4.1)

$$
\omega_{\mathrm{r}}=\omega_{\mathrm{v}}\left[\left(1-\frac{\alpha_{\mathrm{e}}+\alpha_{\mathrm{v}}}{4 d^{3}}\right) /\left(1-\frac{\alpha_{\mathrm{e}}}{4 d^{3}}\right)\right]^{1 / 2}
$$

Thus the frequency is always lowered. If the molecule is far from the surface, the shift is

$$
\Delta \omega \equiv \omega_{\mathrm{r}}-\omega_{\mathrm{v}} \cong-\left(\alpha_{\mathrm{v}} / 8 d^{3}\right) \omega_{\mathrm{v}} .
$$

The observed shifts for adsorbed molecules are of a few percent. Since electronic polarizabilities are tpyically some fraction of the molecular volume, it is clear that these shifts can be fitted with spacings that are some multiple of the molecular radius. For an adsorbed molecule, however, the spacing should be somewhat smaller, which means that the shifts predicted by this simple argument are probably rather too large. More important, there are obvious effects that should change this simple picture. Among these are finite molecular size and nonlocal response of the metal, both of which might be expected to 
modify significantly the above results. The question therefore is to what extent is the picture of an electromagnetic shift arising from a molecule interacting with its image valid. We try to give an answer in this chapter.

In section 4.1 we show how to take into account nonlocal response of the metal and finite molecular size for a molecule whose electromagnetic response is characterized by its multiple polarizabilities. We also calculate the response function, which is the analog of the expression (4.3) above. In section 4.2 we discuss the calculation of the multipole polarizabilities and give results for several simple electronic models. Then in section 4.3 , just as above, we identify the resonant frequency with a pole of the response function. This pole occurs at a complex frequency, the imaginary part arising from loss in the metal, so we find not only a shift but also a broadening. In section 4.4 we discuss other contributions to the shift, the mechanical shift due to the adsorption bond to the metal and the "chemical" shift due to hybridization of the molecular orbitals with metallic electron states. We then combine these shifts with the electromagnetic shift and broadening and compare them with experimental results. Finally, in section 4.5 we give a short discussion of the coverage dependence of the shift. We have chosen $\mathrm{CO}$ on copper for our model calculations, since the most widely studied system from an experimental point of view is $\mathrm{CO}$ on various noble metals. Experimental work on this problem has been reviewed by Sheppard and Nguyen [68].

\subsection{Response of a spherical molecule above a nonlocal metal}

We consider here the problem of a spherical molecule placed above a nonlocal metal surface, as shown in fig. 18. We want to calculate the induced dipole moment in the molecule due to an applied field $\boldsymbol{E}_{0} \mathrm{e}^{\mathrm{i} \omega t}$. We assume the molecular radius $\boldsymbol{a}$ and the molecule-metal spacing $d$ are both small compared with the wavelength of emitted light $\lambda=2 \pi c / \omega \varepsilon_{1}^{1 / 2}$. This assumption has two consequences: the applied field may be taken as uniform, and we may use the quasistatic approximation in calculating the response. In this approximation, the nonlocal response of the metal surface can be described by the P-polarized reflection coefficient, as discussed in section 2.2, while the response of the molecule can be described by its multipole polarizabilities, introduced in another context in section 3.6. The spirit of the discussion in this section will therefore be that the molecule and the metal are described by their

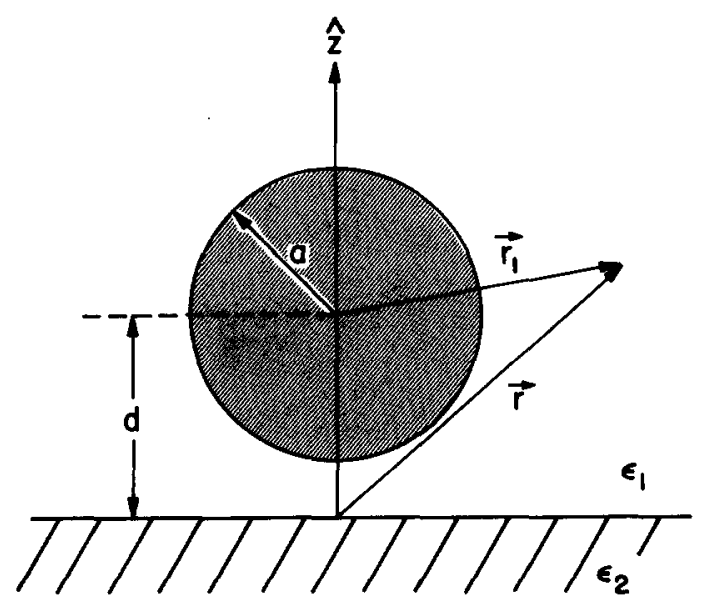

Fig. 18. Geometry for a spherical molecule above a metal. 
responses to external fields, and we calculate only with the fields outside of both. The calculation of these responses, multipole polarizabilities for the molecule and reflection coefficients for the metal, is viewed as a problem to be discussed separately.

As in our previous discussion we choose the metal surface to be the $x y$ plane with the metal filling the region below the plane. The sphere (molecule) is centered on the $z$ axis at a height $d$ above the plane as shown in fig. 18. In the quasistatic approximation the electric field in the region outside the molecule and above the metal (the unhatched region in fig. 18) is obtained from a potential of the form

$$
\Phi=-\boldsymbol{E}_{0} \cdot \boldsymbol{r}+\sum_{l=1}^{\infty} \sum_{m=-l}^{l} B_{m l} r_{1}^{-l-1} P_{l}^{m}\left(\cos \theta_{1}\right) \exp \left(\mathrm{im} \phi_{1}\right)+\int \mathrm{d} \boldsymbol{p} B(\boldsymbol{p}) \exp [\mathrm{i}(\boldsymbol{p} \cdot \boldsymbol{\rho}-p z)]
$$

where

$$
\boldsymbol{r}=\boldsymbol{\rho}+z \hat{\boldsymbol{z}}=\boldsymbol{r}_{1}+\hat{\boldsymbol{z}} d
$$

and $\theta_{1}, \phi_{1}$ are the polar angles of $\boldsymbol{r}_{1}$. In (4.5) the first term is the potential of the uniform applied field, the second term is the sum over the potentials due to the induced multipoles in the sphere, and the third term is the integral over the potential of the induced field from the metal corresponding to $p$, the $x y$ projection of the propagation vector. The coefficients $B_{l m}$ and $B(p)$ are determined from the requirement that just above the metal surface this potential must be a superposition of potentials of the form

$$
\Phi_{\boldsymbol{p}} \propto \exp (\mathrm{ip} \cdot \boldsymbol{\rho})\left(-\mathrm{e}^{p z}+\boldsymbol{r}_{12}^{\mathrm{P}} \mathrm{e}^{-p z}\right)
$$

corresponding to the form (2.37) of the quasistatic electric field, while just outside the sphere the potential must be a superposition of potentials of the form

$$
\Phi_{l m} \propto\left(-r_{1}^{l}+\frac{\alpha_{l}}{\varepsilon_{1} r_{1}^{l+1}}\right) P_{l}^{m}\left(\cos \theta_{1}\right) \exp \left(\mathrm{i} m \phi_{1}\right)
$$

as in (3.42). The similarity of these two forms should be obvious. In either case the first term within the parentheses represents the potential of an applied field due to external sources, while the second represents the potential of the field due to induced sources within the medium. In the planar case the response is characterized by the reflection coefficient $r_{12}^{\mathrm{P}}=r_{12}^{\mathrm{P}}(p, \omega)$, while in the spherical case it is characterized by the multipole polarizabilities $\alpha_{l}=\alpha_{l}(\omega)$. In this present section, as we have stated, we view these quantities as given.

In order to apply these conditions we use a pair of mathematical identities. The first is

$$
\exp (\mathrm{i} p \cdot \boldsymbol{\rho}-p z)=\mathrm{e}^{-p d} \sum_{l=0}^{\infty} \sum_{m=-l}^{l} \frac{\mathrm{i}^{m}\left(-p r_{1}\right)^{l}}{(l+m) !} P_{l}^{m}\left(\cos \theta_{1}\right) \exp \left[\mathrm{im}\left(\phi_{1}-\phi\right)\right],
$$

where $\phi$ is the azimuthal angle of $p$. This identity, which is valid for $z>0$, is used to express the potential of the reflected field from the metal in terms of incident multipole fields at the molecule. The second identity is 


$$
r_{1}^{-l-1} P_{l}^{m}\left(\cos \theta_{1}\right) \exp \left(\mathrm{i} m \phi_{1}\right)=\frac{\mathrm{i}^{m}(-)^{l-m}}{2 \pi(t-m) !} \int \mathrm{d} p p^{l-1} \exp [\mathrm{i} \boldsymbol{\rho} \cdot \boldsymbol{\rho}+p(z-d)] \exp (\mathrm{i} m \phi)
$$

which holds for $z<d$ and serves to express the potential of the induced multipole in the molecule in terms of plane waves incident upon the surface. A brief derivation of these identities is given in appendix A.

To apply the condition just above the metal surface, we use (4.10) in the terms within the sum in (4.5) to write

$$
\Phi=-\boldsymbol{E}_{0} \cdot \boldsymbol{r}+\int \mathrm{d} \boldsymbol{p} \exp (\mathrm{i} \boldsymbol{p} \cdot \boldsymbol{\rho})\left[-\sum_{l=1}^{\infty} \sum_{m=-l}^{l} B_{l m} \frac{(-\mathrm{i})^{m}(-p)^{l-1} \mathrm{e}^{-p d+i m \phi}}{2 \pi(l-m) !} \mathrm{e}^{p z}+B(p) \mathrm{e}^{-p z}\right]
$$

Requiring the integrand to be of the form (4.7) we find

$$
B(p)=r_{12}^{\mathrm{P}} \mathrm{e}^{-p d} \sum_{l=1}^{\infty} \sum_{m=-l}^{l} B_{l m} \frac{(-\mathrm{i})^{m}(-p)^{l-1} \mathrm{e}^{\mathrm{i} m \phi}}{2 \pi(l-m) !}
$$

Note that the uniform field does not enter this condition, since a uniform field always can satisfy the boundary conditions.

Next consider the condition just outside the sphere. We put (4.9) in the integral in (4.5) to write

$$
\Phi=-\boldsymbol{E}_{0} \cdot \boldsymbol{r}_{1}+\sum_{l=1}^{\infty} \sum_{m=-l}^{l}\left[\frac{\mathrm{i}^{m}}{(l+m) !} \int \mathrm{d} p B(p)(-p)^{l} \mathrm{e}^{-\mathrm{i} m \phi} r_{1}^{l}+B_{l m} r_{1}^{-l-1}\right] P_{l}^{m}\left(\cos \theta_{1}\right) \exp \left(\mathrm{i} m \phi_{1}\right),
$$

where we have dropped the constant terms (from $l=0$ in (4.9) and from replacing $\boldsymbol{r}=\boldsymbol{r}_{1}+\hat{\boldsymbol{r}} \boldsymbol{d}$ in the first term), which only adjust the potential at the molecule. We can write

$$
\boldsymbol{E}_{0} \cdot \boldsymbol{r}_{1}=\hat{\boldsymbol{z}} \cdot \boldsymbol{E}_{0} r_{1} P_{1}\left(\cos \theta_{1}\right)-\frac{1}{2}(\hat{\boldsymbol{x}}-\mathrm{i} \hat{\boldsymbol{y}}) \cdot \boldsymbol{E}_{0} r_{1} P_{1}^{1}\left(\cos \theta_{1}\right) \mathrm{e}^{\mathrm{i} \phi_{1}}+(\hat{\boldsymbol{x}}+\mathrm{i} \hat{\boldsymbol{y}}) \cdot \boldsymbol{E}_{0} r_{1} P_{1}^{-1}\left(\cos \theta_{1}\right) \mathrm{e}^{-\mathrm{i} \phi_{1}},
$$

where we have used $P_{1}=\cos \theta_{1}, P_{1}^{1}=-2 P_{1}^{-1}=-\sin \theta$. Using this expression in (4.13) and requiring the result to be a sum of potentials of the form (4.8), we find

$$
\frac{\varepsilon_{1}}{\alpha_{l}} B_{l m}+\frac{\mathrm{i}^{m}}{(l+m) !} \int \mathrm{d} p B(p)(-p)^{l} \mathrm{e}^{-\mathrm{i} m \phi}=\delta_{l, 1}\left[\hat{\boldsymbol{z}} \cdot \boldsymbol{E}_{0} \delta_{m, 0}-\frac{1}{2}(\hat{\boldsymbol{x}}-\mathrm{i} \hat{\boldsymbol{y}}) \cdot \boldsymbol{E}_{0} \delta_{m, 1}+(\hat{\boldsymbol{x}}+\mathrm{i} \hat{\boldsymbol{y}}) \cdot \boldsymbol{E}_{0} \delta_{m,-1}\right]
$$

This relation together with the relation (4.12) determines the coefficients in the expression (4.5) for the pontential.

To find the coefficients $B_{l m}$ we put $B(p)$ given by (4.12) in the integral in the relation (4.15). In the resulting integral the integration over $\phi$, the azimuthal angle of $p$, gives a factor of $2 \pi \delta_{m, m^{\prime}}$, where $m^{\prime}$ is the summation variable in (4.12). For each $m$ we thus obtain an infinite set of coupled inhomogeneous linear equations for the coefficients $B_{l m}$, which we write in the form 


$$
\frac{\varepsilon_{1}}{\alpha_{l}} B_{l m}+\sum_{l^{\prime}=1}^{\infty}(-)^{l+l^{\prime}+1}\left(\begin{array}{c}
l+l^{\prime} \\
l+m
\end{array}\right) F_{l+l^{\prime}} B_{l^{\prime} m}=\delta_{l, 1}\left[\hat{\boldsymbol{z}} \cdot \boldsymbol{E}_{0} \delta_{m, 0}-\frac{1}{2}(\hat{\boldsymbol{x}}-\mathrm{i} \hat{\boldsymbol{y}}) \cdot \boldsymbol{E}_{0} \delta_{m, 1}+(\hat{\boldsymbol{x}}+\mathrm{i} \hat{\boldsymbol{y}}) \cdot \boldsymbol{E}_{0} \boldsymbol{\delta}_{m,-1}\right],
$$

where where $\left(\begin{array}{c}N \\ M\end{array}\right)$ is the binomial coefficient and

$$
F_{L}(\omega, d) \equiv \frac{1}{L !} \int_{0}^{\infty} \mathrm{d} p r_{12}^{\mathrm{P}}(p, \omega) p^{L} \mathrm{e}^{-2 p d}
$$

Since these equations are uncoupled for different values of $m$ (a consequence of our assumption of spherical symmetry for the molecule), we see immediately

$$
B_{l m}=0, \quad m \neq 0, \pm 1 .
$$

For $m=0, \pm 1$ we can write these equations in a simpler form if we introduce a new set of coefficients $A_{l m}$ given by

$$
B_{l, 0}=(-)^{l+1} \frac{1}{\varepsilon_{1}} \hat{\boldsymbol{z}} \cdot \boldsymbol{E}_{0} A_{l, 0}, \quad B_{l, 1}=(-)^{l} \frac{1}{2 \varepsilon_{1}}(\hat{\boldsymbol{x}}-\mathrm{i} \hat{\boldsymbol{y}}) \cdot \boldsymbol{E}_{0} A_{l, 1}, \quad B_{l,-1}=(-)^{l+1} \frac{1}{\varepsilon_{1}}(\hat{\boldsymbol{x}}+\mathrm{i} \hat{\boldsymbol{y}}) \cdot \boldsymbol{E}_{0} A_{l,-1} .
$$

The coupled equations (4.16) can then be written in the form

$$
\sum_{l^{\prime}=1}^{\infty} M_{l, l^{\prime}}^{m} A_{l^{\prime}, m}=\delta_{l, 1}
$$

where

$$
M_{l, l^{\prime}}^{m}=\frac{1}{\alpha_{l}} \delta_{l, l^{\prime}}-\frac{1}{\varepsilon_{1}}\left(\begin{array}{l}
l+l^{\prime} \\
l+m
\end{array}\right) F_{l+l^{\prime}}
$$

The solution of these equations is expressed by Cramer's rule [69]:

$$
A_{l m}=\operatorname{cof}\left(M_{1, l}^{m}\right) / \operatorname{det}\left(M^{m}\right)
$$

where the denominator is the determinant of the matrix $M^{m}$ whose elements are the $M_{l, l^{\prime}}^{m}$, and the numerator is the cofactor of the $1, l$ element in this matrix. In our case, where the matrices are infinite, the meaning of (4.22) is the limit as $n \rightarrow \infty$ of the corresponding ratio formed with the $n$th truncation of $M^{m}$, the $n \times n$ matrix in the upper left hand corner. A brief discussion of convergence of infinite determinants is given by Whittaker and Watson [70].

The potential of the induced dipole moment in the sphere corresponds to the $l=1$ terms in the sum in (4.5). That is,

$$
\boldsymbol{\mu} \cdot \boldsymbol{r}_{1} / \varepsilon_{1} r_{1}^{3}=\sum_{m=-1}^{1} B_{1, m}\left(1 / r_{1}^{2}\right) P_{1}^{m}\left(\cos \theta_{1}\right) \exp \left(i m \phi_{1}\right)
$$


From this expression we can identify the components of $\boldsymbol{\mu}$, using (4.14). Then, using (4.19) we can write these components in terms of the coefficients $A_{1, m}$. Finally, we note $A_{1,-1}=A_{1,1}$ as we see from (4.22) and the fact that the matrix $M^{-m}$ is the transpose of the matrix $M^{m}$, which in turn is clear from the expression (4.21) for the matrix elements. The result can be written in the form

$$
\boldsymbol{\mu}(\omega, d)=A_{1,0} \hat{z} \cdot E_{0} \hat{z}+A_{1,1}\left(E_{0}-\hat{z} \cdot E_{0} \hat{z}\right)
$$

This is our desired expression for the induced dipole moment in the sphere. When the reflection coefficient $r_{12}^{\mathrm{P}}(p, \omega)$ and the multipole polarizabilities $\alpha_{l}(\omega)$ are given, the quantities $A_{1,0}$ and $A_{1,1}$ are therefore calculated by the following prescription. First calculate the integrals $F_{L}$ given by (4.17). Then form the matrix $M^{m}$ whose elements are given by (4.21). The quantities $A_{1, m}$ are then given by (4.22). In practice this prescription is carried out for successive truncations of the matrix, increasing the size of the truncation until convergence is obtained.

The quantities $A_{1,1}$ and $A_{1,0}$ are generalized susceptibilities, in the sense of linear response theory [71]. As functions of the complex variable $\omega$ they are analytic and have no zeros in the upper half-plane. Moreover, $-i \omega A_{1, m}(\omega)$ is what is termed a positive function, with real part positive for $\omega$ in the upper half-plane as well on the real axis. The analytic continuation of the generalized susceptibilities into the lower half-plane will in general have poles, which are associated with normal modes of the system. The positions of these poles give the complex frequencies of the normal modes, the real part being the frequency and twice the (negative) imaginary part being the decay rate of the mode. We use these general ideas in section 4.3 to obtain expressions for the shift and broadening of a molecular vibration mode.

\subsection{Multipole polarizabilities}

We discuss here the calculation of the multipole polarizabilities for some simple electronic systems. The aim will be to gain insight into the structure of these quantities for real molecules and to obtain for special models explicit expressions useful for computations based on the formalism derived in the previous section.

For a one-electron system with a non-degenerate spherically symmetric ground state, the $2^{l}$-pole polarizability is given by the quantum mechanical formula

$$
\alpha_{l}=e^{2} \sum_{\mathrm{i}}\left|\left\langle\psi_{\mathrm{i}}, r^{l} P_{l}(\cos \theta) \psi_{\mathrm{g}}\right\rangle\right|^{2}\left(\frac{1}{\mathscr{E}_{\mathrm{i}}-\mathscr{E}_{\mathrm{g}}-\hbar \omega}+\frac{1}{\mathscr{E}_{\mathrm{i}}-\mathscr{E}_{\mathrm{g}}+\hbar \omega}\right)
$$

where the sum is over intermediate states $\psi_{\mathrm{i}}$ with energy $\mathscr{E}_{\mathrm{i}}$ and $\psi_{\mathrm{g}}$ is the ground state with energy $\mathscr{C}_{\mathrm{g}}$. This is the direct generalization of the well-known Kramers-Heisenberg dispersion formula for the dipole polarizability [72]. In fact, this formula is not as useful as a basis for calculation as it appears, since except for the harmonic oscillator the sum converges slowly. However, we do see from this formula that there will be resonances in $\alpha_{l}$ corresponding to the Bohr frequencies $\omega= \pm\left(\mathscr{E}_{\mathrm{i}}-\mathscr{E}_{\mathrm{g}}\right) / \hbar$. In this chapter we are interested in the case where there is such a resonance, corresponding to a vibrational frequency of an adsorbed molecule. However, we will postpone the discussion of this case and consider first the case where the frequency of the exciting radiation is well below all the Bohr frequencies and the polarizability becomes the static polarizability.

For the static polarizabilities a more useful approach than the Kramers-Heisenberg formula is to 
solve explicitly for the perturbed wave function [73]. According to (3.42) the multipole polarizabilities give the response of the molecule to an applied multipole field. We therefore consider a perturbed Hamiltonian of the form

$$
H=H_{0}+\operatorname{er}^{l} P_{l}(\cos \theta)
$$

where the second term is $-e$ times the potential of the applied multipole field and

$$
H_{0}=-\left(\hbar^{2} / 2 m\right) \nabla^{2}+U(r)
$$

is the molecular Hamiltonian corresponding to a single electron in a spherically symmetric potential $U(r)$. The unperturbed ground state is a spherically symmetric eigenstate of $H_{0}$ :

$$
H_{0} \psi_{\mathrm{g}}=\mathscr{E}_{\mathrm{g}} \psi_{\mathrm{g}}, \quad \psi_{\mathrm{g}}=\psi_{\mathrm{g}}(r)
$$

The perturbed eigenvalue problem is

$$
\left(H_{0}+\operatorname{er}^{l} P_{l}\right) \psi=\mathscr{E} \psi
$$

Expanding

$$
\psi=\psi_{\mathrm{g}}+\psi^{(1)}+\cdots, \quad \mathscr{E}=\mathscr{E}_{\mathrm{g}}+\mathscr{E}^{(1)}+\cdots,
$$

the first-order equation is

$$
\left(H_{0}-\mathscr{E}_{\mathrm{g}}\right) \psi^{(1)}=-\operatorname{er}^{l} P_{l} \psi_{\mathrm{g}}+\mathscr{E}^{(1)} \psi_{\mathrm{g}}
$$

The first-order energy is

$$
\mathscr{E}^{(1)}=\int \mathrm{d} r \psi_{\mathrm{g}}^{*}(r) \operatorname{er}^{l} P_{l}(\cos \theta) \psi_{\mathrm{g}}(r)=0
$$

since $l>0$. If we put therefore

$$
\psi^{(1)}(r)=R_{l}(r) P_{l}(\cos \theta)
$$

and use (4.27), the first-order equation (4.31) becomes

$$
\left[\frac{\hbar^{2}}{2 m}\left(-\frac{1}{r^{2}} \frac{\mathrm{d}}{\mathrm{d} r} r^{2} \frac{\mathrm{d}}{\mathrm{d} r}+\frac{l(l+1)}{r^{2}}\right)+U(r)-\mathscr{C}_{\mathrm{g}}\right] R_{l}=-\mathrm{e} r^{l} \psi_{\mathrm{g}}
$$

This inhomogeneous differential equation for the perturbed radial wave function $R$ is sometimes called the Sternheimer equation [74].

If we have solved the Sternheimer equation, the induced charge density is

$$
-e n_{\mathrm{ind}}(r)=-e\left(|\psi|^{2}-\left|\psi_{\mathrm{g}}\right|^{2}\right)=-e\left(\psi_{\mathrm{g}}^{*} R_{l}+\psi_{\mathrm{g}} R_{l}^{*}\right) P_{l}+\cdots
$$


The corresponding induced electrostatic potential in a medium with dielectric constant $\varepsilon_{1}$ is

$$
\Phi_{\mathrm{ind}}(\boldsymbol{r})=\int \mathrm{d} \boldsymbol{r}^{\prime} \frac{-e n_{\mathrm{ind}}\left(\boldsymbol{r}^{\prime}\right)}{\varepsilon_{1}\left|\boldsymbol{r}-\boldsymbol{r}^{\prime}\right|} \sim \sum_{l=0}^{\infty} \frac{-e}{\varepsilon_{1} r^{I+1}} \int \mathrm{d} \boldsymbol{r}^{\prime} \boldsymbol{r}^{\prime l} P_{l}\left(\hat{\boldsymbol{r}} \cdot \hat{\boldsymbol{r}}^{\prime}\right) n_{\mathrm{ind}}\left(\boldsymbol{r}^{\prime}\right)
$$

where we have used the well-known expansion of $\left|\boldsymbol{r}-\boldsymbol{r}^{\prime}\right|$ for $r>\boldsymbol{r}^{\prime}$ [75]. Using the addition theorem for spherical harmonics [75],

$$
P_{l}\left(\hat{\boldsymbol{r}} \cdot \hat{\boldsymbol{r}}^{\prime}\right)=P_{l}(\cos \theta) P_{l}\left(\cos \theta^{\prime}\right)+2 \sum_{m=1}^{l} \frac{(l-m) !}{(l+m) !} P_{l}^{m}(\cos \theta) P_{l}^{m}\left(\cos \theta^{\prime}\right) \cos m\left(\phi-\phi^{\prime}\right),
$$

the form (4.35) for $n_{\text {ind }}$, and the orthogonality of the spherical harmonics [75], we find the induced potential is of the form corresponding to the defining relation (3.42),

$$
\Phi_{\text {ind }}(r) \sim\left(\alpha_{l} / \varepsilon_{1} r^{l+1}\right) P_{l}(\cos \theta)
$$

where the multipole polarizability is given by

$$
\alpha_{l}=-\frac{4 \pi e}{2 t+1} \int_{0}^{\infty} \mathrm{d} r r^{l+2}\left[\psi_{\mathrm{g}}^{*} R_{l}(r)+\psi_{\mathrm{g}}(r) R_{l}(r)^{*}\right]
$$

The Sternheimer equation has been used extensively as the basis for numerical calculation of the multipole polarizabilities for small $l$. For a recent example, see ref. [76]. We are more interested, however, in the behavior for all $l$, for which exact results for simple models are instructive.

For a hydrogenic atom

$$
V(r)=-Z e^{2} / r, \quad \psi_{\mathrm{g}}(r)=\pi^{-1 / 2}\left(Z a_{0}\right)^{-3 / 2} \exp \left(-r / Z a_{0}\right)
$$

where $a_{0}=\hbar^{2} / m e^{2}$. The solution of (4.34) is

$$
R_{l}(r)=-\frac{m e}{\hbar^{2}}\left(Z a_{0}\right)^{2}\left(\frac{1}{l} r^{l}+\frac{1}{l+1} \frac{r^{l+1}}{Z a_{0}}\right) \psi_{\mathrm{g}}(r)
$$

Putting this in (4.39) we find

$$
\alpha_{l}=Z\left(Z a_{0}\right)^{2 l+1} \frac{(2 l+1) !(l+2)}{2^{2 l} l}
$$

We can write this in the form

$$
\alpha_{l}=\frac{(2 l+1) !(l+2)}{18 l(12)^{l-1}} \alpha_{1}\left\langle r^{2}\right\rangle^{l-1}
$$

where $\alpha_{1}$ is the dipole polarizability and 


$$
\left\langle r^{2}\right\rangle=\int \mathrm{d} r \psi_{\mathrm{g}}^{*} r^{2} \psi_{\mathrm{g}}=3\left(Z a_{0}\right)^{2}
$$

is the mean square radius of the ground state.

In the same way for the harmonic oscillator

$$
\begin{aligned}
& V(r)=\frac{1}{2} m \omega_{0}^{2} r^{2} \\
& \psi_{\mathrm{g}}(r)=\left(m \omega_{0} / \pi \hbar\right)^{3 / 4} \exp \left(-m \omega_{0} r^{2} / 2 \hbar\right)
\end{aligned}
$$

The solution of (4.34) is

$$
R_{l}(r)=-\left(e / l \hbar \omega_{0}\right) r^{l} \psi_{\mathrm{g}}(r)
$$

and

$$
\alpha_{l}=\frac{(2 l-1) ! !}{l 3^{l-1}} \alpha_{1}\left\langle r^{2}\right\rangle^{l-1}
$$

where $(2 l-1) ! !=1 \times 3 \times 5 \cdots(2 l-1)$ and

$$
\alpha_{1}=e^{2} / m \omega_{0}^{2}, \quad\left\langle r^{2}\right\rangle=3 \hbar / 2 m \omega_{0} .
$$

Note that $\alpha_{1}$ is the familiar static dipole polarizability of the oscillator.

As a final example of a quantum mechanical model for which the solution is simple we consider the infinite spherical well for which

$$
V(r)=\left\{\begin{array}{ll}
0, & 0<r<a \\
\infty, & a<r<\infty
\end{array}, \quad \psi_{\mathrm{g}}(r)=(2 \pi a)^{-1 / 2} \sin (\pi r / a) / r\right.
$$

The solution of (4.34) is

$$
R_{l}(r)=-\frac{1}{l+1} \frac{m e}{\hbar^{2}}\left(\frac{a}{2 \pi^{3}}\right)^{1 / 2}\left[r^{l} \cos (\pi r / a)+a^{l} j_{l}(\pi r / a) / j_{l}(\pi)\right]
$$

where $j_{l}$ is the spherical Bessel function. Putting this in (4.39) we find

$$
\alpha_{l}=\frac{6 g(l)}{(l+1)(2 l+1) \pi^{2 l-2} g(1)} \alpha_{1} a^{2 l-2}
$$

where

$$
g(l)=\int_{0}^{\pi} \mathrm{d} y\left[y^{2 l+1} \sin y \cos y+\pi^{l} y^{l+1} \sin y j_{l}(y) / j_{l}(\pi)\right]
$$


and

$$
\alpha_{1}=\left(1 / 3 \pi^{2}+1 / 4 \pi^{2}\right) a^{4} / a_{0}
$$

The integrals $g(l)$ are elementary and can be performed explicitly, although this soon becomes tedious as $l$ increases. However, for $l \geqslant 3$ they are well approximated by their asymptotic form:

$$
g(l) \sim 3 \pi^{2 l+5} /\left[(2 l+1)^{2}+\pi^{2}\right]\left[(2 l+1)^{2}+(2 \pi)^{2}\right] .
$$

These results for quantum mechanical models are all to be compared with the multipole polarizability of a uniform dielectric sphere:

$$
\alpha_{l}=\frac{\varepsilon_{1} l\left(\varepsilon_{3}-\varepsilon_{1}\right)}{l \varepsilon_{3}+(l+1) \varepsilon_{1}} a^{2 l+1}=\frac{3 l}{2 l+1+(l-1) \alpha_{1} / \varepsilon_{1} a^{3}} \alpha_{1} a^{2 l-2},
$$

where $\varepsilon_{3}$ is the dielectric constant of the sphere and $a$ its radius.

In fig. 19 we compare the multipole polarizabilities as calcualted from the above formulas for the various models. Here we plot the dimensionless quantity $\alpha_{l} / \alpha_{1} a^{2 l-2}$ as a function of $l$ for each model. For the spherical square well and dielectric sphere models $a$ is the sphere radius, while for the hydrogenic atom and oscillator models $a$ is the rms radius of the ground state $\left(a=\left\langle r^{2}\right\rangle^{1 / 2}\right)$. The results for the various models are strikingly different, but the reasons for this can be easily understood. For the hydrogenic atoms and oscillator models the integral (4.39) is for large $l$ dominated by the region of large $r$. This is so because of the high power of $r$ appearing explicitly in the integrand of (4.39) and also because of the corresponding high power of $r$ multiplying $\psi_{\mathrm{g}}$ in the expressions (4.41) and (4.47) for $R_{l}$. In this region of large $r$, however, the perturbing potential in (4.29) is no longer small compared with the molecular potential $U(r)$, and perturbation theory cannot be expected to be valid. On the other hand, there is no such criticism of the predictions for the spherical square well model since the infinite barrier at $r=a$ limits the range of integration. There, however, for $l$ large the high power of $r$ in the integrand

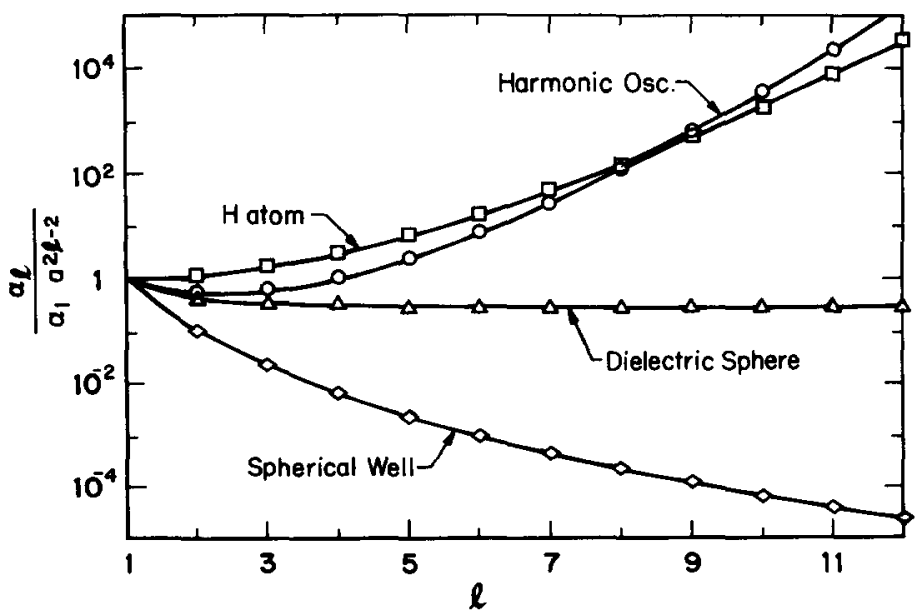

Fig. 19. Multipole polarizabilities for various molecular models. 
of (4.39) emphasizes the neighborhood of $r=a$, where $\psi_{\mathrm{g}}(r)$ vanishes. This is the reason for the rapid fall-off as shown in fig. 19 for this model. Intermediate between the rapid increase shown for the hydrogenic atom and oscillator models and the rapid decrease shown for the spherical square well model is the smooth transition to an asymptotic value shown for the dielectric sphere model. Primarily because we feel that the extreme behavior of the other models is largely of calculational origin and reflects artificial features of the models rather than essential physics, we choose for most of our calculations the dielectric sphere model to represent the multipole polarizabilities of a finite molecule. We should emphasize that this is a crude approximation which probably underestimates the $\alpha_{l}$, at least for intermediate values of $l$. There is a need for a careful, critical study of methods for calculating multipole polarizabilities for realistic molecular models.

We now turn to the problem of including dynamical effects in the multipole polarizabilities, effects which are essential if we are to describe the resonance associated with molecular vibrations. The key to how we do this is the phenomenological form (4.1) for the dipole polarizability of a molecule with a single vibrational mode. There $\alpha_{\mathrm{e}}$ represents the polarizability due to the electronic cloud of the molecule and is frequency independent while the additional polarizability due to the vibrational mode has a resonance structure. This is the form of the polarizability which arises from a model of the molecule as a polarizable sphere (the electron cloud) with a point-like vibrating dipole at the center [77]. We accordingly extend this model to the higher multipoles, which will be entirely of electronic origin and of the form (4.56) with $\alpha_{1}=\alpha_{\mathrm{e}}$. That is we write

$$
\alpha_{l}(\omega)=\frac{3 l \alpha_{\mathrm{e}} a^{2 l-2}}{2 l+1+(l-1) \alpha_{\mathrm{e}} / \varepsilon_{1} a^{3}}+\frac{\alpha_{\mathrm{v}}}{1-\left(\omega / \omega_{\mathrm{v}}\right)^{2}} \delta_{l, 1} .
$$

As we have emphasized above, this is a crude model, but it does describe the essential physical features of a polarizable electron cloud surrounding the vibrating bond.

Efrima and Metiu [78] have used a very different finite-size molecular model. They model the vibrational polarizability by a pair of point charges oscillating about a finite separation and the electronic polarizability by a polarizable point at the center of the molecule. Their model and ours might be viewed as two extremes. We treat the vibrator as point-like and ascribe all finite-size effects to the electron cloud. They treat the electron cloud as point-like and ascribe finite size effects to the separation of the ion cores. We believe that for calculating the electromagnetic effects, our model is more appropriate, since the outer regions of the molecule are in fact occupied by the valence electrons. Our calculations on this and similar problems show that all finite-size effects disappear rapidly as the molecule is moved away from the surface. Thus, the vibrator, being more distant than the electron cloud, should be more point-like.

\subsection{Shift and broadening of the vibrational mode}

We consider here the case of a molecule near the surface responding to an electric field oriented perpendicular to the surface. The example we have in mind is $\mathrm{CO}$ adsorbed on $\mathrm{Cu}$ with the molecule oriented perpendicular to the surface and with the $\mathrm{C}$ atom bonded to the surface. We describe the molecular response by the multipole polarizabilities given by (4.57) and the metal response by the quasistatic reflection coefficient $r_{12}^{\mathrm{P}}$. The complex vibrational frequency $\omega_{\mathrm{r}}$ of the mode will therefore be associated with a pole in the generalized susceptibility $A_{1,0}(\omega)$, which according to (4.24) describes the induced dipole moment in the molecule. The idea here is very simple: the induced dipole moment 
satisfies the equation

$$
\left[A_{1,0}(\omega)\right]^{-1} \mu=E_{0}
$$

and there will therefore be a free motion in zero applied field (i.e., a normal mode) at $\omega=\omega_{\mathrm{r}}$ if and only if

$$
\left[A_{1,0}\left(\omega_{\mathrm{r}}\right)\right]^{-1}=0
$$

(i.e., $A_{1,0}(\omega)$ has a pole at $\omega=\omega_{\mathrm{r}}$ ).

In our case the condition can be rearranged so as to make the vibrational parameters explicit. Note that (4.57) can be written in the form

$$
\frac{1}{\alpha_{l}(\omega)}=\frac{2 l+1+(l-1) \alpha_{\mathrm{e}} / \varepsilon_{1} a^{3}}{3 l \alpha_{\mathrm{e}} a^{2 l-2}} \frac{\alpha_{\mathrm{v}}}{\alpha_{\mathrm{e}}} \frac{\omega_{\mathrm{v}}^{2}}{\left(\alpha_{\mathrm{e}}+\alpha_{\mathrm{v}}\right) \omega_{\mathrm{v}}^{2}-\alpha_{\mathrm{e}} \omega^{2}} \delta_{l, 1}
$$

Putting this in the expression (4.21) for the elements of the matrix $M^{m}$ we see that the matrix is the same as that for a dielectric sphere except for the 1,1 element in the upper left-hand corner. If therefore in the expression (4.22) for $A_{1,0}$ we expand $\operatorname{det}\left(M^{0}\right)$ according to elements in the first row, we readily see that

$$
\left[A_{1,0}(\omega)\right]^{-1}=\left[A_{1,0}^{\mathrm{e}}(\omega)\right]^{-1}-\frac{\alpha_{\mathrm{v}}}{\alpha_{\mathrm{e}}} \frac{\omega_{\mathrm{v}}^{2}}{\left(\alpha_{\mathrm{e}}+\alpha_{\mathrm{v}}\right) \omega_{\mathrm{v}}^{2}-\alpha_{\mathrm{e}} \omega^{2}}
$$

where $A_{1,0}^{\mathrm{e}}(\omega)$ is the generalized electronic susceptibility corresponding to just the electronic part of the molecular response. That is, $A_{1,0}^{\mathrm{e}}(\omega)$ is obtained from (4.22) in which in the matrix elements (4.21) the quantity $1 / \alpha_{l}$ is given by the first term in (4.60), corresponding to $\alpha_{v}=0$. The advantage of this rearrangement is that $A_{1,0}^{\mathrm{e}}(\omega)$ is a slowly varying function of $\omega$ [its frequency dependence comes only from the metal response described by the $F_{L}(\omega)$ in (4.21)] with no sharp structure near $\omega=\omega_{\mathrm{v}}$. Therefore in the condition (4.59), and provided $\omega_{\mathrm{r}}$ is near $\omega_{\mathrm{v}}$, we can replace $A_{1,0}^{\mathrm{e}}\left(\omega_{\mathrm{r}}\right) \rightarrow A_{1,0}^{\mathrm{e}}\left(\omega_{\mathrm{v}}\right)$ and solve explicitly to find

$$
\omega_{\mathrm{r}}^{2}=\omega_{\mathrm{v}}^{2}\left\{1+\left(\alpha_{\mathrm{v}} / \alpha_{\mathrm{e}}\right)\left[1-A_{1,0}^{\mathrm{e}}\left(\omega_{\mathrm{v}}\right) / \alpha_{\mathrm{e}}\right]\right\}
$$

The real part of $\omega_{\mathrm{r}}$ is interpreted as the shifted vibrational frequency, the imaginary part as the half-width. Provided the shift is small we obtain for the relative shift the expression

$$
\left.\Delta \omega / \omega_{\mathrm{v}} \equiv \operatorname{Re}\left\{\omega_{\mathrm{r}}-\omega_{\mathrm{v}}\right) / \omega_{\mathrm{v}}\right\} \approx\left(\alpha_{\mathrm{v}} / 2 \alpha_{\mathrm{e}}\right) \operatorname{Re}\left\{1-A_{1,0}^{\mathrm{e}}\left(\omega_{\mathrm{v}}\right) / \alpha_{\mathrm{e}}\right\}
$$

and for the relative half-width $\dagger$

$$
\gamma / 2 \omega_{\mathrm{v}} \equiv-\operatorname{Im}\left\{\omega_{\mathrm{r}} / \omega_{\mathrm{v}}\right\} \approx\left(\alpha_{\mathrm{v}} / 2 \alpha_{\mathrm{e}}^{2}\right) \operatorname{Im}\left\{A_{1,0}^{\mathrm{e}}\left(\omega_{\mathrm{v}}\right)\right\}
$$

\footnotetext{
† Here $\gamma$ is the decay rate which is also the full-width at half maximum of the resonance. In ref. [77] this same symbol was used for the
} half-width. 
A special case of these results is that in which finite molecular size is neglected and the molecule is treated as point-like with polarizability given by (4.1). Setting $\alpha_{l}=0$ for $l>1$, we find from (4.22)

$$
\left[A_{1,0}^{\mathrm{e}}(\omega)\right]^{-1}=\frac{1}{\alpha_{\mathrm{e}}}-\frac{2}{\varepsilon_{1}} F_{2}(\omega, d) \quad \text { (point model) }
$$

Putting this into (4.63) and (4.64) we get

$$
\frac{\Delta \omega}{\omega_{\mathrm{v}}} \approx-\alpha_{\mathrm{v}} \operatorname{Re}\left\{\frac{F_{2}\left(\omega_{\mathrm{v}}, d\right)}{\varepsilon_{1}-2 \alpha_{\mathrm{e}} F_{2}\left(\omega_{\mathrm{v}}, d\right)}\right\}, \quad \frac{\gamma}{2 \omega_{\mathrm{v}}} \approx \alpha_{\mathrm{v}} \operatorname{Im}\left\{\frac{F_{2}\left(\omega_{\mathrm{v}}, d\right)}{\varepsilon_{1}-2 \alpha_{\mathrm{e}} F_{2}\left(\omega_{\mathrm{v}}, d\right)}\right\} \quad \text { (point model). }
$$

It is of interest to compare this expression for the width with the corresponding expression for the decay rate obtained in chapter 3 . According to general principles the two should be the same [1]. To make the comparison we first should drop the term involving $F_{2}$ in the denominator of (4.66) since that corresponds to a renormalization of the fluctuating dipole moment due to image fields from the metal, which was neglected in chapter 3. Then we get the same result if in the relation (3.25), i.e., $\gamma=\mathscr{P} / \hbar \omega$, we use the quasistatic expression (3.22) for the power dissipated and provided we put $\mu_{\|}=0$ and $\mu_{\perp}^{2}=2 \hbar \omega_{v} \alpha_{v}$. This last identity is obtained if we interpret $\mu_{\perp}$ as twice the quantum transition dipole moment from the first excited state to the ground state of the oscillator corresponding to the vibrational mode, i.e., $\mu_{\perp}=-2 e\left(\hbar / 2 m \omega_{v}\right)^{1 / 2}$, and interpret $\alpha_{v}$ as the corresponding static polarizability of the oscillator, i.e., $\alpha_{\mathrm{v}}=e^{2} / m \omega_{\mathrm{v}}^{2}$. Thus the apparently very different methods of this chapter and of chapter 3 do indeed give identical results for the decay rates.

If we further specialize to represent the metal as a medium with a local dielectric constant $\varepsilon_{2}(\omega)$, then, still in the quasistatic approximation, $r_{12}^{\mathrm{P}}=\left(\varepsilon_{2}-\varepsilon_{1}\right) /\left(\varepsilon_{2}+\varepsilon_{1}\right)$ and $F_{2}=r_{12}^{\mathrm{P}} / 8 d^{3}$. The results $(4.66)$ then become

$$
\begin{aligned}
& \Delta \omega / \omega_{\mathrm{v}} \approx-\frac{1}{2} \alpha_{\mathrm{v}} \operatorname{Re}\left\{\left(\varepsilon_{2}-\varepsilon_{1}\right) /\left[4 \varepsilon_{1}\left(\varepsilon_{2}+\varepsilon_{1}\right) d^{3}-\alpha_{\mathrm{e}}\left(\varepsilon_{2}-\varepsilon_{1}\right)\right]\right\}, \\
& \gamma / 2 \omega_{\mathrm{v}} \approx \frac{1}{2} \alpha_{\mathrm{v}} \operatorname{Im}\left\{\left(\varepsilon_{2}-\varepsilon_{1}\right) /\left[4 \varepsilon_{1}\left(\varepsilon_{2}+\varepsilon_{1}\right) d^{3}-\alpha_{\mathrm{e}}\left(\varepsilon_{2}-\varepsilon_{1}\right)\right] \quad\right. \text { (point model, local metal). }
\end{aligned}
$$

This result for the shift reduces far from the surface to the simple result (4.4a) if we take $\varepsilon_{2}=\infty$, corresponding to a perfect reflector, and set $\varepsilon_{1}=1$.

In fig. 20 are shown numerical results calculated on the basis of the expressions (4.63) and (4.64) and plotted vs. the molecular separation. The metal reflectivity was calculated using the SCIB model with the Lindhard dielectric function, as described in chapter 2, i.e., using (2.30) in (2.39). The distance $d$ is from the center of the sphere to the infinite barrier. The parameters chosen were those appropriate to the case of $\mathrm{CO}$ adsorbed on $\mathrm{Cu}$. For the molecule the parameters are $\alpha_{\mathrm{e}}=2.6 \AA^{3}, \alpha_{\mathrm{v}}=0.16 \AA^{3}$ and $\omega_{\mathrm{v}}=2143 \mathrm{~cm}^{-1}[79,80]$. The radius $a$ was chosen to be $1 \AA$. For the metal the parameters were determined as follows. From optical (infrared) data [81] $\varepsilon_{\mathrm{b}}=6.5$ and $\omega_{\mathrm{p}}=8.73 \mathrm{eV}$ were determined from a least squares fit to the form $\varepsilon(\omega)=\varepsilon_{\mathrm{b}}-\omega_{\mathrm{p}}^{2} / \omega^{2}$. The electron scattering rate $\hbar \nu=0.05 \mathrm{eV}$ was determined from more recent optical data [82]. The Fermi wavevector was determined from the electron density of $\mathrm{Cu}$ using $k_{\mathrm{F}}=\left(3 \pi^{2} n\right)^{1 / 3}=1.36 \AA^{-1}$. The Fermi velocity was determined from the slope of the calculated conduction band [83] at the Fermi surface, $v_{\mathrm{F}}=(\mathrm{d} \mathscr{E} / \mathrm{d} k)_{k=k_{\mathrm{F}}}=0.008 c$. The results using these parameters are shown in the curves labeled $\varepsilon_{\mathrm{b}}=6.5$ in fig. 20 . The results are insensitive to the choice of $\nu$ and $k_{\mathrm{F}}$. On the other hand, they are strongly dependent upon the choice of 


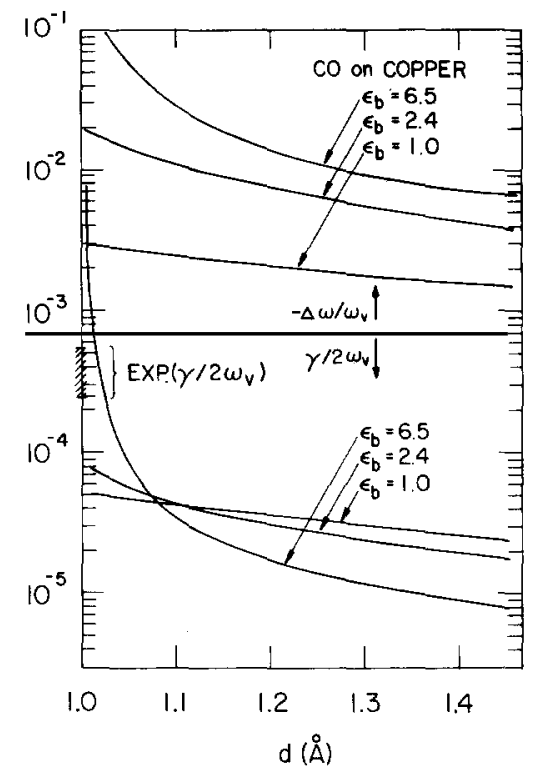

Fig. 20. Results calculated using the SCIB model for the electromagnetic shift $(\Delta \omega)$ and broadening $(\gamma)$ of $\mathrm{CO}$ on $\mathrm{Cu}$ showing the effect of the core polarization term $\varepsilon_{\mathbf{b}}$. The molecule is modeled as a dielectric sphere, and the metal parameters are given in the text. After ref. [77].

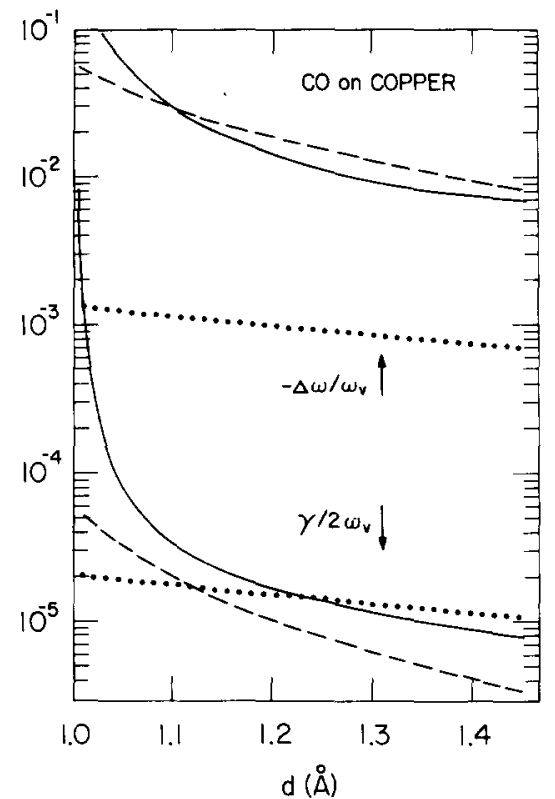

Fig. 21. Calculated results for the shift and broadening from various models. The solid curve is the $\varepsilon_{\mathrm{b}}=6.5$ curve from fig. 20 . The dashed curve is for a local metal with a point-like molecule. The dotted curve is for a nonlocal metal with a point-like molecule but neglecting core polarization and image effects. After ref. [77].

$\varepsilon_{\mathrm{b}}$. To show the importance of this choice, results for $\varepsilon_{\mathrm{b}}=2.4$ and $\varepsilon_{\mathrm{b}}=1$ are also shown. The choice $\varepsilon_{\mathrm{b}}=2.4$ corresponds to using the Clausius-Mossotti formula, $\left(\varepsilon_{\mathrm{b}}-1\right) /\left(\varepsilon_{\mathrm{b}}+2\right)=4 \pi n \alpha / 3$, where for $\mathrm{Cu}$ the ion core density $n=4 /(3.61 \AA)^{3}$ while the ionic core polarizability is chosen to be the cube of the ion core radius, $\alpha=(0.96 \AA)^{3}[84]$. The choice $\varepsilon_{\mathrm{b}}=1$ corresponds to a jellium model with no core polarization. It is clear from these results that the jellium model gives predictions for the shift and broadening which are very different from those corresponding to a more realistic choice of $\varepsilon_{\mathrm{b}}$. In this connection we should emphasize that, although the plots in fig. 20 are carried out to rather large values of $d$, finite molecular size has been taken into account and the molecule should be positioned so as to be nearly touching the surface, i.e., near the left-hand side of the plot.

In fig. 21 the shift and broadening are plotted in the same way as in fig. 20, but now the purpose is to show the dependence upon finite molecular size and nonlocal metal response. The solid curves are a repetition of the $\varepsilon_{\mathrm{b}}=6.5$ curves from fig. 20 , corresponding to a finite-size molecule above a nonlocal metal. The dashed curves correspond to a point molecule above a local metal with the same parameters, i.e., as calculated from (4.67) with $\varepsilon_{1}=1$ and $\varepsilon_{2}=\varepsilon_{\mathrm{b}}-\omega_{\mathrm{p}}^{2} / \omega(\omega+\mathrm{i} \nu)$. The curves corresponding to a point molecule above a nonlocal metal, i.e., as calculated from (4.66), are not shown since to do so would clutter the figure. They would lie very close to the solid curves for $d>1.1 \AA$, while for smaller $d$ they would continue smoothly, more or less parallel to the dashed curves. The first and most striking conclusion from these results is that finite size effects are large and that they disappear rapidly as the molecule is moved away from the surface. Recall that the radius chosen is $a=1 \AA$, so that the left-hand side of the plots corresponds to the molecule (sphere) just touching the surface. Thus when the molecule is removed only a fraction of its radius from the surface it appears to be point-like. This is in contrast to the effects of nonlocality in the metal, which persist to much greater distances. As pointed 
out in chapter 3 and demonstrated in the calculations shown in fig. 8, nonlocal effects are important even when the molecule is several tens of angstroms from the surface. In this connection we should remark that the rough agreement of the solid and dashed curves for $d \geq 1.1 \AA$ stems from the corresponding agreement of the reflection coefficients for the local and nonlocal metal, which as pointed out in our discussion of fig. 4 in section 3.2, is misleading. The physically correct description of the metal in the region depicted by fig. 21 is the nonlocal one. Finally we remark upon what we can call the image divergence of the shift, which for the naive model of a point-like molecule above a perfectly reflecting metal occurs at $d=\left(\alpha_{\mathrm{e}} / 4\right)^{1 / 3}$, as we see from (4.4). The inclusion of damping in the metal reduces this divergence to a peak at more or less the same position. But in fig. 21 this peak in the dashed curve is out of sight to the left, the part of the curve shown is only rising slowly toward the peak. On the other hand the solid curves in fig. 21 rise rapidly as the molecule is brought close to the surface. This is the residue of the image peak, much reduced but brought to a position just outside the metal surface by the finite size of the molecule.

The dotted curve in fig. 21 is calculated for a point-like molecule above a nonlocal metal but with the neglect of image effects, i.e., dropping the term involving $F_{2}$ in the denominator of (4.66). The metal was described by the jellium model with the same parameters as in the curve labeled $\varepsilon_{b}=1$ in fig. 20 . The purpose here is to show that calculations based on these approximations $[15,85]$ seriously underestimate the broadening and shift, in particular when the molecule is near the surface.

In fig. 22 a comparison is made of calculations based on the SCIB and QIB models to describe the

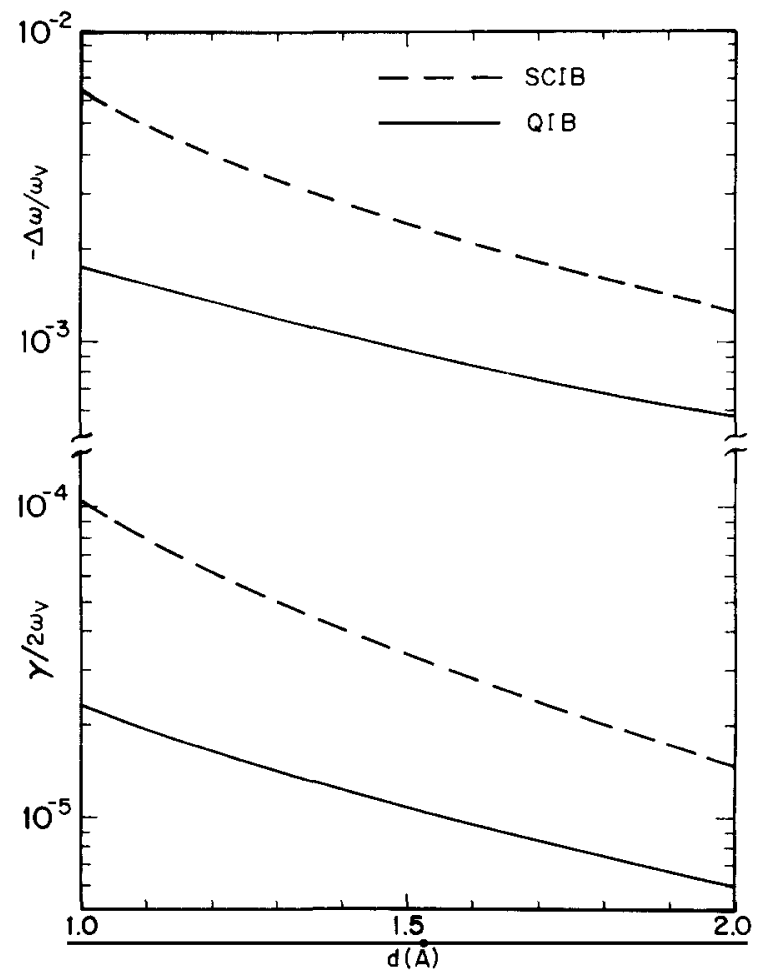

Fig. 22. Comparison between results calculated with the QIB model (solid lines) and the SCIB model (dashed lines) for the shift and broadening of $\mathrm{CO}$ on a free electron metal with no core polarization. The molecule is modeled as in figs. 20 and 21 . The distance $d$ is measured from the infinite barrier. 
metal response. Finite molecular size was taken into account as described above, i.e., the calculations were based on (4.63) and (4.64) with $\alpha_{\mathrm{e}}=2.6 \AA^{3}, \alpha_{\mathrm{v}}=0.16 \AA^{3}$ and $\omega_{\mathrm{v}}=2143 \mathrm{~cm}^{-1}$. For the QIB model the reflection coefficient was calculated as described in section 2.3, for the SCIB model (2.39) was used. In either case the parameters chosen were those of a collisionless free electron gas with the density of $\mathrm{Cu}: \varepsilon_{\mathrm{b}}=1, \nu=0, \hbar \omega_{\mathrm{p}}=10.8 \mathrm{eV}, k_{\mathrm{F}}=1.36 \AA^{-1}$, and $\varepsilon_{1}=1$. The results are unexciting, as could have been anticipated from the corresponding curves labeled $\varepsilon_{b}=1$ in fig. 20. The SCIB model predicts systematically larger shifts and broadening. But this is a reflection largely of the fact that the effective edge of the electron density for the QIB model, the jellium edge, is inside the infinite barrier at $d=-3 \pi / 8 k_{\mathrm{F}} \approx-0.9 \AA$. If the solid curves are translated to the right by this distance, they lie close to the dashed curves. We have seen this phenomenon earlier in connection with fig. 9. Thus we conclude that, after this adjustment, there is little reason to prefer the QIB model over the SCIB model. In view of the importance of the bound electron contribution $\varepsilon_{\mathrm{b}}$, which can be included in the SCIB model, we would argue that it gives overall a more accurate representation of the essential physics of this problem, this despite the fact that it does not realistically describe the smooth transition of the electron density in the surface region.

There have been recent calculations of the broadening, based on methods of the type described in section 2.4, for a free electron gas with a Lang-Kohn [86] electron density profile in the surface region $[85,87]$. The predictions are not much different from those of the QIB model. Again, because of the importance of the bound electron response, we feel that the SCIB model is to be preferred over these more elaborate calculations. We should make clear that these remarks apply to the case of non-freeelectron metals at optical and infrared frequencies.

\subsection{Other contributions to the shift}

In this section we discuss other contributions to the shift and broadening and make a comparison with experimental results. Since our primary purpose in this article is the discussion and critical examination of the electromagnetic interactions, our remarks here will be brief, although we shall try to make them self-contained. We first consider the mechanical contribution to the shift, due to the binding of the molecule to the metal. We then consider the effects of the zero-point motion of the molecule in the binding potential. Finally we make some remarks about the chemical shift, due to changes in the molecular bond orbitals, and then give a brief comparison with some experimental results.

The mechanical shift arises because of the binding of the molecule to the metal surface. To illustrate how it is calculated we consider a simple vibrator model corresponding to the case of $\mathrm{CO}$ bonded in an upright orientation to a metal surface. This is shown in fig. 23 , where $m_{C}$ is the $\mathrm{C}$ mass, $m_{\mathrm{O}}$ the $\mathrm{O}$ mass and $M$ the mass of a metal atom or complex of metal atoms. The force constant $k$ is assumed to be the

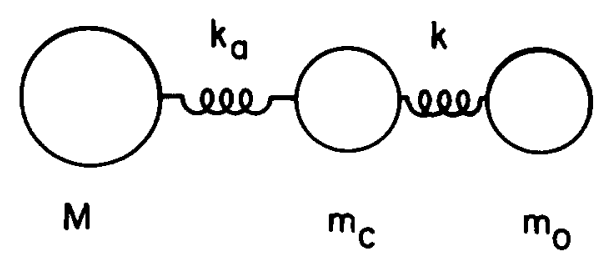

Fig. 23. Coupled mass and spring model used to calculate mechanical shifts. 
same as that of the free molecule, while the adsorption force constant $k_{\mathrm{a}}$ is presumably weak. The normal mode frequencies are roots of the secular equation [88]:

$$
\omega^{4}-\left(\frac{m_{\mathrm{O}} m_{\mathrm{C}}}{m_{\mathrm{O}} m_{\mathrm{C}}} k+\frac{m_{\mathrm{C}}+M}{m_{\mathrm{C}} M} k_{\mathrm{a}}\right) \omega^{2}+\frac{m_{\mathrm{O}}+m_{\mathrm{C}}+M}{m_{\mathrm{O}} m_{\mathrm{C}} M} k k_{\mathrm{a}}=0
$$

The vibrational frequency $\omega_{\mathrm{v}}$ of the free molecule corresponds to $k_{\mathrm{a}}=0$,

$$
\omega_{\mathrm{v}}=\left(\frac{m_{\mathrm{O}}+m_{\mathrm{C}}}{m_{\mathrm{O}} m_{\mathrm{C}}} k\right)^{1 / 2}
$$

For nonzero $k_{\mathrm{a}}$ there will be two roots, $\omega_{+}$and $\omega_{-}$, which satisfy

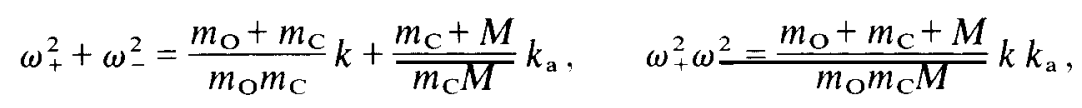

and which we choose such that $\omega_{+}>\omega_{-}$. Now if the force constant $k_{\mathrm{a}}$ is small, corresponding to weak bonding to the metal, the frequency $\omega_{+}$will be near $\omega_{v}$ while $\omega_{-}$will be small. The relation between these three frequencies is obtained by using (4.69) and eliminating $k_{\mathrm{a}}$ between eqs. (4.70). The result can be put in the form

$$
\left(\omega_{+}^{2}-\omega_{v}^{2}\right) / \omega_{\mathrm{v}}^{2}=\mathrm{m}_{\mathrm{O}} M \omega_{-}^{2} /\left[m_{\mathrm{C}}\left(m_{\mathrm{O}}+m_{\mathrm{C}}+M\right) \omega_{\mathrm{v}}^{2}-\left(m_{\mathrm{O}}+m_{\mathrm{C}}\right)\left(m_{\mathrm{C}}+M\right) \omega_{-}^{2}\right] .
$$

The point of this relation is that the frequencies $\omega_{v}$, the vibrational frequency of the free molecule, and $\omega_{-}$, the low frequency of the molecule bouncing against the metal, can be measured, which allows one to predict the shifted frequency $\omega_{+}$in terms of measured quantities. For the case of $\mathrm{CO}$ adsorbed on $\mathrm{Cu}(100)$ the measured value $\omega_{-}=343 \mathrm{~cm}^{-1}$ [89]. Then with $m_{\mathrm{C}}=12, m_{\mathrm{O}}=16$, and $M=\infty$, corresponding to the molecule being bonded to a large metal complex in the surface, we find $\omega_{+}=2182 \mathrm{~cm}^{-1}$. If we take $M=63$ or 65 , corresponding to the molecule bonded to a single $\mathrm{Cu}$ atom, we find $\omega_{+}=2170 \mathrm{~cm}^{-1}$. The mechanical shift is therefore

$$
\Delta \omega \equiv \omega_{+}-\omega_{\mathrm{v}}=33 \pm 6 \mathrm{~cm}^{-1}
$$

Another effect of this mechanical motion that must be considered is the zero-point motion of the molecule bouncing against the surface. Although this effect does not itself cause a shift, it does modify the electromagnetic shift. In the approximation of weak binding, i.e., $k_{\mathrm{a}}$ small, and the mass $M$ large, this motion corresponds to that of a single mass $m_{\mathrm{O}}+m_{\mathrm{C}}$ oscillating with frequency $\omega_{-}$. The corresponding mean square amplitude at temperature $T$ is

$$
\left\langle\Delta z^{2}\right\rangle=\frac{\hbar}{2\left(m_{\mathrm{O}}+m_{\mathrm{C}}\right) \omega_{-}} \operatorname{coth} \frac{\overline{\hbar \omega}_{-}}{2 k_{\mathrm{B}} T} .
$$

At room temperature $k_{\mathrm{B}} T=25 \mathrm{meV}$, and for the above parameters of $\mathrm{CO}$ adsorbed on $\mathrm{Cu}$ we find

$$
\left(\left\langle\Delta z^{2}\right\rangle\right)^{1 / 2} \approx 0.05 \AA \text {. }
$$


In principle the electromagnetic shift and broadening as calculated in section 4.3 should be averaged over this small amplitude. The change in the predictions is quite small.

The chemical shift is the shift arising from changes in the bonding orbitals upon adsorption. In terms of the picture of fig. 23, used to describe the mechanical shift, one would expect that the bonding to the metal represented by the force constant $k_{\mathrm{a}}$ would also change the force constant $k$ from its value in the free molecule. This would give rise to a corresponding change (shift) in the frequency $\omega_{v}$, given by (4.69). Provided this shift is small it can be added to the mechanical shift, and the two in turn added to the electromagnetic shift. There is good evidence from experiments on isotopic varieties of copper carbonyls [90] that this picture of a chemical change in bond strength together with a mechanical shift as given by (4.71) is consistent.

We now make a brief comparison with experimental results, the purpose being to show how, in our opinion, these calculations should be used. First, the broadening should be of electromagnetic origin. $\dagger$ The observed width for $\mathrm{CO}$ adsorbed on $\mathrm{Cu}$ is $\gamma \approx 12-24 \mathrm{~cm}^{-1}$, or $\gamma / 2 \omega_{\mathrm{v}} \approx 3-6 \times 10^{-3}$ [91]. Comparing with the $\varepsilon_{\mathrm{b}}=6.5$ curve in fig. 20 , we see that this width can be fitted if $d \cong 1.03 \AA$. Then for this value of $d, \Delta \omega / \omega_{\mathrm{v}} \cong-0.1$, or $\Delta \omega=-200 \mathrm{~cm}^{-1}$. This shift added to the mechanical shift (4.72) of about $+35 \mathrm{~cm}^{-1}$ and an unknown chemical shift is to be compared with the experimental shift $[92,93]$ of about $-60 \mathrm{~cm}^{-1}$. We find therefore that the chemical shift should be about $+65 \mathrm{~cm}^{-1}$. This positive value for the chemical shift is surprising, since one would expect bonding effects to weaken the bond, leading to a negative chemical shift. Indeed, the experimental evidence on copper carbonyls [90] suggests a chemical shift of about $-160 \mathrm{~cm}^{-1}$. The conclusion we draw from this is not that the electromagnetic contribution to the shift is absent, but that the observed width is probably due to inhomogeneous broadening, corresponding to $\mathrm{CO}$ adsorbed on a variety of sites on the metal surface. In addition, the bonding in $\mathrm{CO}$ adsorbed on copper is probably much different from that in copper carbonyls. We trust that this brief and rough comparison with observation, which is about all that can be justified by the experimental results, has shown the use to which the calculations can be put. In fact a good bit of insight has been gained. The electromagnetic contribution to the shift and broadening is significant. The observed widths can be fitted, but it is probable that the true homogeneous width is rather smaller, corresponding to a less closely bound molecule. It is clear, however, that the electromagnetic shift and broadening are related, and that both must be taken into account.

\subsection{Coverage dependence}

We conclude this chapter with a brief discussion of the effects of surface coverage on the shift and broadening. Our primary goal is to show how to incorporate the results of the previous sections, involving finite-size molecules and nonlocal metals, into calculations of the coverage dependence that have previously only considered point molecules or local metals $[66,67]$. The effect we are concerned with arises from the electromagnetic interactions between the molecules on the surface. It is often referred to as dipolar coupling, since the interaction is dominated by the near fields of the dipoles. When the coverage is so low that the mean spacing of the molecules is comparable to the wavelength, which would require including retardation, the interaction is negligible. For these near fields we can use the quasistatic form, as calculated in section 4.1. On the other hand the interest is in the case of at most moderate coverage, so that the mean spacing of the molecules is large compared with the molecular size. This means that we can determine the fields from the potential (4.5) evaluated in the asymptotic

† Broadening due to phonon emission as the molecule bounces against the surface should be negligible [77]. 
region of large $\rho$. This we do in the next paragraph, where we show that asymptotically the potential (4.1) becomes that of the dipole plus its image as calculated in the local approximation. Then, after making some remarks on this result, we discuss the coverage dependence due to such dipolar interactions.

The potential (4.5) can be written $\Phi=-\boldsymbol{E}_{0} \cdot \boldsymbol{r}+\Phi_{\text {ind }}$, where $\Phi_{\text {ind }}$ is the potential due to the induced charges in the metal and in the molecule. Using (4.12), we write $\Phi_{\text {ind }}$ in the form

$$
\begin{aligned}
\Phi_{\text {ind }}(\boldsymbol{r})= & \sum_{l=1}^{\infty} \sum_{m=-l}^{l} B_{l m}\left\{r_{1}^{-l-1} P_{l}^{m}\left(\cos \theta_{1}\right) \exp \left(\mathrm{im} \phi_{1}\right)\right. \\
& \left.+\frac{(-)^{l+1}(-\mathrm{i})^{m}}{2 \pi(l-m) !} \int \mathrm{d} p r_{12}^{\mathrm{P}}(p) p^{l-1} \exp [\mathrm{i} \boldsymbol{p} \cdot \boldsymbol{\rho}-p(z+d)+\mathrm{i} m \phi]\right\} .
\end{aligned}
$$

We want to evaluate this for $\rho$ large, in which case the integral in each term is dominated by the region near $p=0$ in the integrand. We may therefore replace $r_{12}^{\mathrm{P}}(p) \rightarrow r_{12}^{\mathrm{P}}(0)$ in the integrals and use the identity

$$
\frac{1}{r_{2}^{l+1}} P_{l}^{m}\left(\cos \theta_{2}\right) \exp \left(\mathrm{i} m \phi_{1}\right)=\frac{(\mathrm{i})^{m}}{2 \pi(l-m) !} \int \mathrm{d} p p^{l-1} \exp [\mathrm{i} \boldsymbol{p} \cdot \boldsymbol{\rho}-p(z+d)+\mathrm{i} m \phi]
$$

where, referring to fig. $18, \boldsymbol{r}_{2}=\boldsymbol{\rho}+(z+d) \hat{z}=\boldsymbol{r}_{1}+2 d \hat{z}$ is the vector going from the image of the sphere center in the metal to the field point. This identity is, so to speak, the complement of the identity (4.10) and is derived in the same way. With this we obtain the asymptotic formula

$$
\Phi_{\text {ind }}(r) \sim \sum_{l=1}^{\infty} \sum_{m=-l}^{l} B_{l m}\left[r_{1}^{-l-2} P_{l}^{m}\left(\cos \theta_{1}\right) \exp \left(\mathrm{i} m \phi_{1}\right)+r_{12}^{\mathrm{P}}(0)(-)^{l+m+1} r_{2}^{-l-2} P_{l}^{m}\left(\cos \theta_{2}\right) \exp \left(\mathrm{i} m \phi_{1}\right)\right]
$$

which is valid far from the molecule and above the metal surface. The leading terms are those with $l=1$, and these can be expressed in terms of the dipole moment exactly as in (4.23). The result is that we find

$$
\Phi_{\text {ind }}(\boldsymbol{r}) \sim\left(\boldsymbol{\mu} \cdot \boldsymbol{r}_{1}\right) / \varepsilon_{1} r_{1}^{3}+\left(\boldsymbol{\mu}^{\prime} \cdot \boldsymbol{r}_{2}\right) / \varepsilon_{1} r_{2}^{3}
$$

where $\boldsymbol{\mu}$ is the dipole moment induced in the molecule, given by (4.24), and

$$
\boldsymbol{\mu}^{\prime}=r_{12}^{\mathrm{P}}(0)[2 \boldsymbol{\mu} \cdot \hat{z} \hat{z}-\boldsymbol{\mu}]
$$

is the image dipole. Here, as we have seen in our discussion of the long-wavelength limit in section 2.4,

$$
r_{12}^{\mathrm{P}}(0)=\left(\varepsilon_{2}-\varepsilon_{1}\right) /\left(\varepsilon_{2}+\varepsilon_{1}\right) \text {. }
$$

As a refinement of this result we can take into account the first-order corrections to $r_{12}^{\mathrm{p}}$, given by (2.122), by putting the corrections in the form of an exponential to write 


$$
r_{12}^{\mathrm{P}}(p) \approx r_{12}^{\mathrm{P}}(0) \exp \left\{-2 p \frac{\varepsilon_{1} d_{1}+\varepsilon_{2} d_{1}}{\varepsilon_{1}+\varepsilon_{2}}\right\}
$$

Using this in the integrals in (4.75) and repeating the subsequent argument we are again led to the asymptotic form (4.78) with the image dipole given by (4.79) but with

$$
r_{2}=r_{1}+2\left(d+\frac{\varepsilon_{1} d_{\|}+\varepsilon_{2} d_{\perp}}{\varepsilon_{1}+\varepsilon_{2}}\right) \hat{z}
$$

This simply corresponds to a refined definition of the position of the metal surface. Note, however that $d_{\perp}$ is in general complex.

The result (4.78) is remarkable in the sense that it says that far from the molecule the field is that of a point dipole together with its image in a local metal. This despite the fact that finite molecular size and nonlocality of the metal are important in determining the dipole moment of the molecule itself.

In this connection we should remark upon a recent paper of Feibelman [21], who obtains a result similar in form to (4.78), and with a surface position correction identical to (4.82). But Feibelman's result is for the field of a point molecule far from the surface, while (4.78) is for the field far from a molecule which is itself close to the surface.

Now we consider the effect of dipolar interactions between adsorbed molecules, leading to a coverage dependence of the shift and broadening. The applied electric field at the surface is assumed to be slowly varying and of plane wave form: $\boldsymbol{E}_{0} \exp (\mathrm{i} \boldsymbol{p} \cdot \boldsymbol{\rho})$. The position of the $j$ th molecule is denoted by a vector $\boldsymbol{R}_{j}$ parallel to the surface. The dipole moment induced in this molecule is given by (4.24),

$$
\boldsymbol{\mu}_{j}=A_{1,0} \hat{z} \cdot \boldsymbol{E}\left(R_{j}\right) \hat{z}+A_{1,1}\left[\boldsymbol{E}\left(\boldsymbol{R}_{j}\right)-\hat{z} \cdot \boldsymbol{E}\left(\boldsymbol{R}_{j}\right) \hat{z}\right]
$$

where $\boldsymbol{E}\left(\boldsymbol{R}_{j}\right)$, the total electric field seen by the $j$ th molecule, is the sum of the applied field and the dipolar fields due to the other molecules and their images,

$$
\boldsymbol{E}\left(\boldsymbol{R}_{j}\right)=\boldsymbol{E}_{0} \exp \left(\mathrm{ip} \cdot \boldsymbol{R}_{j}\right)+\sum_{k \neq j}\left(\frac{3 \boldsymbol{\mu}_{k} \cdot \boldsymbol{R}_{i k} \boldsymbol{R}_{j k}-R_{j k}^{2} \boldsymbol{\mu}_{k}}{R_{j k}^{5}}+\frac{3 \boldsymbol{\mu}_{k}^{\prime} \cdot \boldsymbol{R}_{j k}^{\prime} \boldsymbol{R}_{j k}^{\prime}-R_{j k}^{\prime 2} \boldsymbol{\mu}_{k}^{\prime}}{R_{j k}^{\prime 5}}\right)
$$

Here $\boldsymbol{R}_{j k}=\boldsymbol{R}_{j}-\boldsymbol{R}_{k}$ is the displacement of the $j$ th molecule relative to the $k$ th molecule while

$$
\boldsymbol{R}_{j k}^{\prime}=\boldsymbol{R}_{j k}+2 D \hat{z}, \quad D=d+\left(\varepsilon_{1} d_{\|}+\varepsilon_{2} d_{\perp}\right) /\left(\varepsilon_{1}+\varepsilon_{2}\right),
$$

is according to (4.82), the displacement of the $j$ th molecule relative to the image of the $k$ th molecule. The image dipole moment $\boldsymbol{\mu}_{k}^{\prime}=2 \hat{z} \cdot \boldsymbol{\mu}_{k} \hat{z}-\boldsymbol{\mu}_{k}$, in accord with (4.79). The equations (4.83) and (4.84) are to be solved for the $\boldsymbol{\mu}_{j}$. To do this we put

$$
\boldsymbol{\mu}_{j}=\boldsymbol{\mu}(\boldsymbol{p}) \exp \left(\mathrm{ip} \cdot \boldsymbol{R}_{j}\right)
$$

Then inverting (4.83) to express $\boldsymbol{E}\left(\boldsymbol{R}_{j}\right)$ in terms of $\boldsymbol{\mu}_{j}$ and using (4.84) we get a single equation for $\boldsymbol{\mu}(\boldsymbol{p})$ which we can write in the dyadic form

$$
\left[\frac{1}{A_{1,0}} \hat{z} \hat{z}+\frac{1}{A_{1,1}}(\overleftrightarrow{1}-\hat{z} \hat{z})-\overleftrightarrow{S}\right] \cdot \boldsymbol{\mu}(\boldsymbol{p})=\boldsymbol{E}_{0},
$$


where $\overleftrightarrow{1}$ is the unit dyadic and

$$
\stackrel{\leftrightarrow}{S}(\boldsymbol{p})=\sum_{k \neq 1} \exp \left(-p \cdot \boldsymbol{R}_{j k}\right)\left(\frac{3 \boldsymbol{R}_{j k} \boldsymbol{R}_{j k}-R_{j k}^{2} \stackrel{\leftrightarrow}{1}}{R_{j k}^{5}}+\frac{3 \boldsymbol{R}_{j k}^{\prime} \boldsymbol{R}_{j k}^{\prime}-R_{j k}^{\prime 2} \stackrel{\leftrightarrow}{1}}{R_{j k}^{\prime 5}}\right)
$$

The inversion of (4.87) to find an explicit expression for $\mu(p)$ is simple in principle (it is only a set of three coupled equations for the components of $\boldsymbol{\mu}$ ) but clumsy in practice because $\vec{S}$ is not diagonal. However, for the case where $\boldsymbol{E}_{0}=E_{0} \hat{z}$ is directed normal to the surface, which is the case of greatest interest, the result takes the simple form

$$
\mu(p)=\frac{1}{1 / A_{1,0}-S_{z z}} E_{0} \hat{z}
$$

where

$$
S_{z z}(p)=-\sum_{k \neq j} \exp \left(\mathrm{i} p \cdot \boldsymbol{R}_{j k}\right)\left(\frac{1}{R_{j k}^{3}}+\frac{1}{\left(R_{j k}^{2}+4 D^{2}\right)^{3 / 2}}-\frac{12 D^{2}}{\left(R_{j k}^{2}+D^{2}\right)^{5 / 2}}\right) .
$$

In general this sum must be performed numerically. However, a simple case of some interest is that in which the wavelength is infinite $(p=0)$, corresponding to a uniform applied field, and the adsorption sites are filled at random with a low density $n$. In this case we can approximate the sum by an integral and write

$$
\begin{aligned}
S_{z z}(0) & \cong-2 \pi n \int_{a}^{\infty} \mathrm{d} R R\left(\frac{1}{R^{3}}+\frac{1}{\left(R^{2}+D^{2}\right)^{3 / 2}}-\frac{\Gamma D^{2}}{\left(R^{2}+D^{2}\right)^{5 / 2}}\right) \\
& =-2 \pi n\left(1 / a+a^{2} /\left(a^{2}+4 D^{2}\right)^{3 / 2}\right)
\end{aligned}
$$

where $a$ is the nearest-neighbor distance for the adsorbed molecules.

The new vibrational frequency for the adsorbed layer is given by setting the denominator on the right side of (4.89) equal to zero and solving for $\omega_{\mathrm{r}}^{2}$ using (4.61). The result can be written

$$
\omega_{\mathrm{r}}^{2}=\omega_{\mathrm{v}}^{2}\left\{1+\alpha_{\mathrm{v}} / \alpha_{\mathrm{e}}-\alpha_{\mathrm{v}} / \alpha_{\mathrm{e}}^{2}\left[A_{1,0}^{\mathrm{e}}\left(\omega_{\mathrm{v}}\right)^{-1}-S_{z z}\right]\right\}
$$

Since $A_{1,0}^{\mathrm{e}}\left(\omega_{\mathrm{v}}\right)^{-1}$ is generally positive (this is what leads to the negative values of $\Delta \omega / \omega$ shown in figs. 20) and 21) and $S_{z z}$ is generally negative, the coverage-dependent part of the shift will be positive. This is in the opposite direction from that of the metal-induced shift. A positive shift of the vibrational frequency with increasing coverage is usually observed for $\mathrm{CO}$ on various metals, but not in all cases [68].

To demonstrate the use of the formulas derived in this section we next estimate the coverage effect for the example of $\mathrm{CO}$ on $\mathrm{Cu}(111)$ at a coverage of 0.1 of a monolayer. Referring to the $\varepsilon_{b}=6.5$ curve in fig. 20 calculated with the SCIB model, we choose $d=1.3 \AA$ for which the electromagnetic shift at zero coverage is $\Delta \omega / \omega_{\mathrm{v}}=-0.01$. We ignore the imaginary part of $A_{1,0}^{\mathrm{c}}\left(\omega_{\mathrm{v}}\right)$ and determine $\operatorname{Re}\left\{A_{1,0}^{\mathrm{e}}\left(\omega_{\mathrm{v}}\right)^{-1}\right\} \cong 0.29 \AA^{-3}$ from eq. (4.62). The $\mathrm{Cu}(111)$ surface has $a=2.55 \AA$ so that $n=0.018 \AA^{-2}$, 
assuming a monolayer is one $\mathrm{CO}$ molecule per $\mathrm{Cu}$ atom. For $D$ we choose $1.5 \AA$, which yields $S_{z z}=-0.058 \AA^{-3}$. The result is rather insensitive to the choice of $D$, but in general $D$ should be on the order of 0.1 to $0.3 \AA$ greater than $d$, even for the SCIB model with a large $\varepsilon_{\mathbf{b}}$. Putting these values into eq. (4.92) and comparing with the result for zero coverage $\left(S_{z z}=0\right)$, we find a $+14 \mathrm{~cm}^{-1}$ shift. We can conclude from this example that the magnitude of the dipolar coupling shift at fractional monolayer coverages is similar to, but of course of opposite sign from, the electromagnetic shift on a bare surface. At 0.5 monolayers a shift of $+10^{2} \mathrm{~cm}^{-1}$ is not unreasonable. The magnitudes of the coverage-dependent shifts calculated here are comparable to those observed experimentally [68]. However, direct comparisons of such calculations with experiment are complicated by several experimental realities: the adsorbed molecules may tend to form islands; there may be a coverage-dependent change in the chemical bonding; and different atomic adsorbtion sites may become filled at higher coverages, which could give abrupt changes in the shifts.

\section{Raman scattering at metal surfaces}

In this chapter we consider the problem of Raman scattering by molecules at or near a metal surface. During the last decade it has been established experimentally that a number of molecules adsorbed on certain metal surfaces have Raman scattering cross sections exceeding their nominal values by as much as 5 or 6 orders of magnitude. This phenomenon is referred to as the Surface Enhanced Raman Scattering (SERS) effect. Its discovery has stimulated major efforts to explain and to exploit the effect. In particular it has had an important impact on the field of surface science. Unlike most other surface probes, such as inelastic electron scattering or Auger electron spectroscopy, which are restricted to high vacuum studies, SERS has the potential for studying molecules in a variety of interfacial environments: metal-gas, metal-liquid, and metal-dielectric. It is sensitive to monolayer concentrations, and the Raman spectra are molecular specific. Thus electrochemical or catalytic reactions on a metal surface could be monitored in situ. Excellent general reviews of experimental and theoretical work on SERS have been given by Otto [94], Metiu [95], and Van Duyne [96], and in addition more detailed reviews are contained in the book edited by Chang and Furtak [97]. We will not attempt to duplicate those reviews here. Instead we discuss several aspects of SERS that can be understood on the basis of classical electromagnetism. Although there is ample evidence that "chemical" effects are also needed to explain SERS, the electromagnetic effects we discuss are always present and must be taken into account.

In the first section of this chapter we consider the image enhancement effect on a smooth surface. Calculations of this effect based on the model system of pyridine on $\mathrm{Ag}$ are presented. In subsequent sections we consider effects produced by rough or structured surfaces.

\subsection{Image enhancement}

The image enhancement effect was one of the first mechanisms offered as an explanation of SERS. In its simplest form, as originally discussed by King et al. [98] and by Efrima and Metiu [99], the molecule is treated as a polarizable point spaced a distance $d$ above a metal with local dielectric constant $\varepsilon_{2}$. An applied macroscopic field $\boldsymbol{E}_{0} \exp \left(-\mathrm{i} \omega_{0} t\right)$ polarizes the molecule inducing a dipole moment $\mu \exp \left(-\mathrm{i} \omega_{0} t\right)$. This dipole has an image in the metal a distance $d$ below the surface given by

$$
\boldsymbol{\mu}_{\text {image }}=\left[\left(\varepsilon_{2}-\varepsilon_{1}\right) /\left(\varepsilon_{2}+\varepsilon_{1}\right)\right](2 \boldsymbol{\mu} \cdot \hat{z} \hat{z}-\mu) .
$$


The image dipole produces a field at the molecule given by

$$
\boldsymbol{E}_{\text {image }}=\left(1 / 8 \varepsilon_{1} d^{3}\right)\left(3 \boldsymbol{\mu}_{\text {image }} \cdot \hat{z} \hat{z}-\boldsymbol{\mu}_{\text {image }}\right),
$$

which also polarizes the molecule. The net dipole induced in the molecule is given by

$$
\boldsymbol{\mu}\left(\omega_{0}\right)=\alpha_{1}\left(\boldsymbol{E}_{0}+\boldsymbol{E}_{\text {image }}\right)
$$

where $\alpha_{1}$ is the dipole polarizability, which we take to be a scalar. Solving these equations for $\mu\left(\omega_{0}\right)$, we obtain

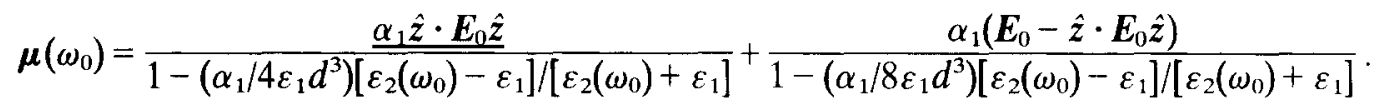

The image enhancement effect is associated with the near vanishing of the denominators in these equations, which can typically occur for distances of $1-2 \AA$.

We next calculate this effect for Raman scattering. To simplify the discussion we take the applied field to be normal to the surface $\left(\boldsymbol{E}_{0}=E_{0} \hat{z}\right)$. The Raman scattering process is pictured as follows. The applied field at $\omega_{0}$, the pump frequency, induces the dipole moment $\boldsymbol{\mu}\left(\omega_{0}\right)$ oscillating at the same frequency. Due to the intrinsic nonlinearities of the molecular motion, the free motion of an internal vibrational mode of frequency $\omega_{\mathrm{v}}$ can mix with this driven motion at the pump frequency to give a dipolar motion at the Raman frequency $\omega_{\mathrm{R}}=\omega_{0}-\omega_{\mathrm{v}} \dagger \dagger$ The dipole induced at the Raman frequency $\mu\left(\omega_{\mathrm{R}}\right)$ should therefore be proportional to $\mu\left(\omega_{0}\right)$ with a proportionality constant $c_{\mathrm{R}}$, which in the absence of the chemical effects is assumed to be unchanged by the presence of the metal. There will also be an image field from the Raman dipole at $\omega_{\mathrm{R}}$, thus in analogy with (5.3),

$$
\mu\left(\omega_{\mathrm{R}}\right)=c_{\mathrm{R}} \mu\left(\omega_{0}\right)+\frac{\alpha_{1} \varepsilon_{2}\left(\omega_{\mathrm{R}}\right)-\varepsilon_{1}}{4 \varepsilon_{1} d^{3} \varepsilon_{2}\left(\omega_{\mathrm{R}}\right)+\varepsilon_{1}} \mu\left(\omega_{\mathrm{R}}\right) .
$$

Solving for $\mu\left(\omega_{\mathrm{R}}\right)$ and using (5.4), we find

$$
\mu\left(\omega_{\mathrm{R}}\right)=c_{\mathrm{R}} \alpha_{1} E_{0}\left[1-\frac{\alpha_{1}}{4 \overline{\varepsilon_{1} d^{3}}} \frac{\varepsilon_{2}\left(\omega_{0}\right)-\varepsilon_{1}}{\varepsilon_{2}\left(\omega_{0}\right)+\varepsilon_{1}}\right]^{-1}\left[1-\frac{\alpha_{1}}{4 \overline{\varepsilon_{1} d^{3}}} \frac{\varepsilon_{2}\left(\omega_{\mathrm{R}}\right)-\varepsilon_{1}}{\varepsilon_{2}\left(\omega_{\mathrm{R}}\right)+\varepsilon_{1}}\right]^{-1} .
$$

The Raman signal is proportional to $\left|\mu\left(\omega_{\mathrm{R}}\right)\right|^{2}$, which far from the metal is given by $\left(c_{\mathrm{R}} \alpha_{1} E_{0}\right)^{2}$. Therefore the gain $G$ induced by the surface can be written

$$
G=\left|1-\frac{\alpha_{1}}{4 \overline{\varepsilon_{1} d^{3}}} \frac{\varepsilon_{2}\left(\omega_{0}\right)-\varepsilon_{1}}{\varepsilon_{2}\left(\omega_{0}\right)+\varepsilon_{1}}\right|^{-2}\left|1-\frac{\alpha_{1}}{4 \overline{\varepsilon_{1} d^{3}}} \frac{\varepsilon_{2}\left(\omega_{\mathrm{R}}\right)-\varepsilon_{1}}{\varepsilon_{2}\left(\omega_{\mathrm{R}}\right)+\varepsilon_{1}}\right|^{-2}
$$

Since the Raman shift is small, the two factors in this expression are nearly equal, and the quantity $G$ will exhibit a narrow peak when plotted vs. $d$, whose maximum value is given approximately by

$\dagger$ The dipole induced at the sum frequency $\omega_{\mathrm{R}}=\omega_{0}+\omega_{v}$ will be negligible as long as $\hbar \omega_{v} \gg k T$. 


$$
G_{\max }=\left\{\frac{\operatorname{Re}\left[\left(\varepsilon_{2}-\varepsilon_{1}\right) /\left(\varepsilon_{2}+\varepsilon_{1}\right)\right]}{\operatorname{Im}\left[\left(\varepsilon_{2}-\varepsilon_{1}\right) /\left(\varepsilon_{2}+\varepsilon_{1}\right)\right]}\right\}^{4} .
$$

In the same approximation the distance $d_{\max }$ at which the peak occurs is given by

$$
d_{\max }=\left\{\frac{\alpha_{1}}{4 \varepsilon_{1}} \operatorname{Re}\left(\frac{\varepsilon_{2}-\varepsilon_{1}}{\varepsilon_{2}+\varepsilon_{1}}\right)\right\}^{1 / 3}
$$

Note that the explicit $\omega$-dependence has been dropped in the last two equations, the assumption being that we may evaluate $\varepsilon_{2}(\omega)$ at $\omega_{0}$ or $\omega_{\mathrm{R}}$. For the case of pyridine adsorbed on $\mathrm{Ag}$ in an upright orientation typical parameters are $\hbar \omega_{0}=2 \mathrm{eV}, \varepsilon_{2}=-19+0.67 \mathrm{i}$, and $\alpha_{1}=11.3 \AA^{3}$, which yields $G_{\max }=$ $5 \times 10^{9}$ at $d_{\max }=1.46 \AA$. Allowing for the fact that the $\omega$-dependence of $\varepsilon_{2}(\omega)$ prevents both factors in eq. (5.7) from being resonant at exactly the same distance reduces $G_{\max }$ to $2 \times 10^{8}$ for a $1000 \mathrm{~cm}^{-1}$ Raman shift. This is still a very large number.

This simplest version of the image enhancement model can be criticized on the basis of its neglect of several important physical phenomena and its requirement of a precise positioning of the molecule. However, it is consistent with the general trends observed for SERS on various metals, which indicate that metals with smaller $\operatorname{Im}\left(\varepsilon_{2}\right)$ show larger gains. Thus, Ag shows the largest SERS gains, Au and Cu show lesser gains that decrease drastically as the frequency approaches the interband absorption region, and transition metals show little or no SERS gains.

We next consider the modifications produced by nonlocal effects, still treating the molecule as point-like. Here we can use the results of chapter 4. The expression (5.4) is the special case for point-like molecules above a local metal of the general result (4.24). Similarly, if we interpret $c_{\mathrm{R}} \mu\left(\omega_{\mathrm{R}}\right) / \alpha_{1}$ as an effective applied field at $\omega_{\mathrm{R}}$, we see that the solution of (5.5) is also the same special case. Moreover, the form of the generalized susceptibility $A_{1,0}$ for the case in which we are now interested, a point-like molecule above a nonlocal metal, is given by eq. (4.65) in which we replace $\alpha_{\mathrm{e}}$ by $\alpha_{1}$. Therefore, the result is to replace expression (5.7) for the gain by

$$
G=\left|1-2 \alpha_{1} F_{2}\left(\omega_{0}, d\right) / \varepsilon_{1}\right|^{-2}\left|1-2 \alpha_{1} F_{2}\left(\omega_{\mathrm{R}}, d\right) / \varepsilon_{1}\right|^{-2}
$$

where $F_{2}(\omega, d)$ is given by eq. (4.17). In the same approximation used for the results (5.8) and (5.9), $G_{\max }=\left[\operatorname{Re}\left(F_{2}\right) / \operatorname{Im}\left(F_{2}\right)\right]^{4}$ and $d_{\max }$ is given implicitly by $2 \alpha_{1} \operatorname{Re}\left[F_{2}(\omega, d)\right]=\varepsilon_{1}$. The qualitative effects of nonlocality are twofold. Screening reduces $\operatorname{Re}\left(F_{2}\right)$ thus decreasing $G_{\max }$ and $d_{\max }$, while the introduction of electron-hole excitations increases $\operatorname{Im}\left(F_{2}\right)$, further reducing $G_{\max }$. These results are summarized in fig. 24, where we show nonlocal point-molecule results based on the SCIB model on the left compared with local point-molecule results on the right. Although there are sizable quantitative changes between the local and nonlocal results shown here, the essential feature of a large narrow peak in the plot of $G$ vs. $d$ is preserved.

The SCIB calculations in fig. 24 use $\varepsilon_{\mathrm{b}}=3.6$ as determined by fitting Ag optical data. The presence of a core polarization term of about this magnitude (or greater) is an essential feature of the metal response. Only then does the image enhancement peak occur at a physically reasonable distance, i.e., $d \geqslant 1 \AA$. The primary effect of the core polarization term is to provide an unscreened $\operatorname{Re}\left(r^{p}\right)$ that does not vanish as $p \rightarrow \infty$. If there is no core polarization $\left(\varepsilon_{\mathrm{b}}=1=\varepsilon_{1}\right)$, then $r^{p} \rightarrow 0$ as $p \rightarrow \infty$ and the position for maximum gain becomes much smaller. For example, calculations with the SCIB model for a free electron gas $\left(\varepsilon_{\mathrm{b}}=1\right)$ with the same electron density and optical properties as $\mathrm{Ag}$ yield an image 


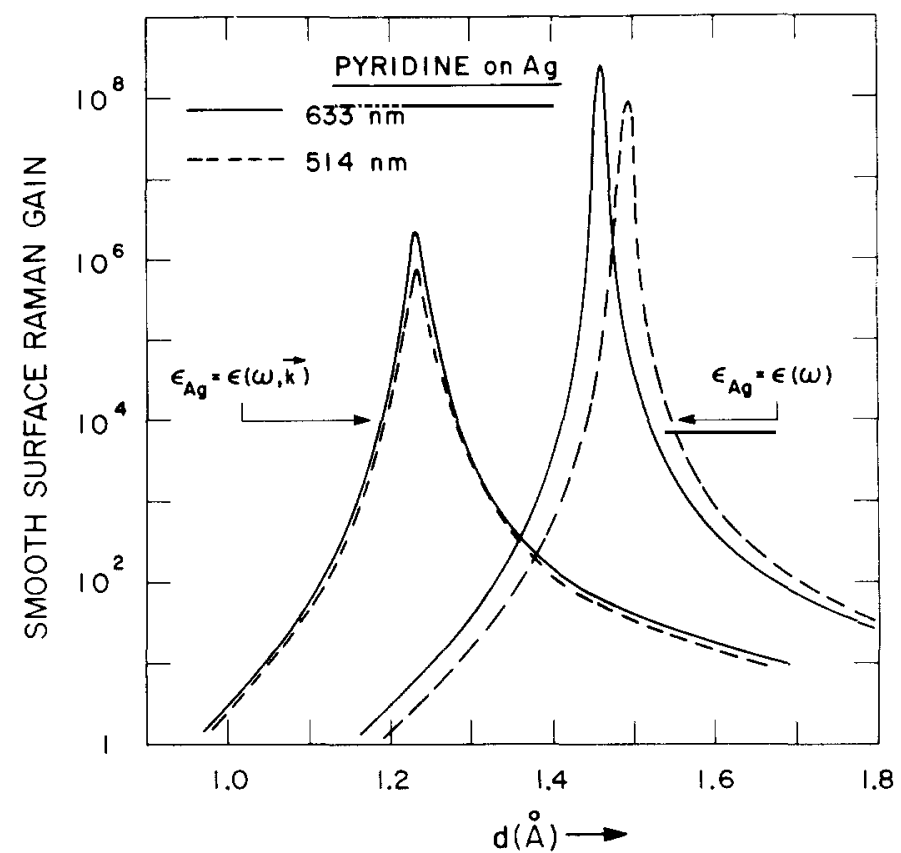

Fig. 24. Image enhancement Raman gain for a point dipole. The curves on the right are calculated for a local metal using eq. (5.7), those on the left for a nonlocal metal using the SCIB model with eq. (5.10). The Ag parameters are the same as those for fig. 4 . The Raman shift is $1000 \mathrm{~cm}^{-1}$. After ref. [42].

enhancement peak at $d_{\max }=0.7 \AA$, which is too small for a molecule such as pyridine [43]. For the same parameters we have likewise found that there is no significant image enhancement effect in the QIB model (which requires $\varepsilon_{\mathrm{b}}=1$ ), as long as the molecule is constrained to be outside the infinite barrier. We can also expect from these results that models such as the one discussed by Feibelman [21] would also show very small image enhancement for pyridine outside a free electron gas.

We now add finite molecular size to the problem. Since the spacings required for maximum gain are comparable to the molecular size, one would expect on intuitive grounds alone that finite size would be important. The expression for the SERS image enhancement gain is obtained from the general result (4.24) for the dipolar response of a finite molecule. As indicated above, the expression (5.4) and the solution of (5.5) are special cases of this general result. Therefore, using (4.24), the image enhancement gain for the case of a finite molecule above a nonlocal surface is given by

$$
G=\alpha_{1}^{-4}\left|A_{1,0}\left(\omega_{\mathrm{R}}, d\right) A_{1,0}\left(\omega_{0}, d\right)\right|^{2},
$$

where $A_{1,0}(\omega, d)$ is given by eq. (4.22). Note that this equation is a general result expressing the image enhancement gain in terms of the as yet unspecified reflection coefficients for the metal and multipole polarizabilities for the molecule.

In fig. 25 we show sample calculations that use the same SCIB model for the metal as was used in fig. 24 , but that treat the pyridine molecule as either a dielectric sphere (solid lines) or an infinite spherical well (dashed lines). In both cases the dipole polarizability is $11.3 \AA^{3}$. The infinite spherical well "molecule" shows a gain close to that of a point-like molecule at the center of the sphere. This is not surprising, since for this model the higher multipole polarizabilities are small, as shown in fig. 19. The 


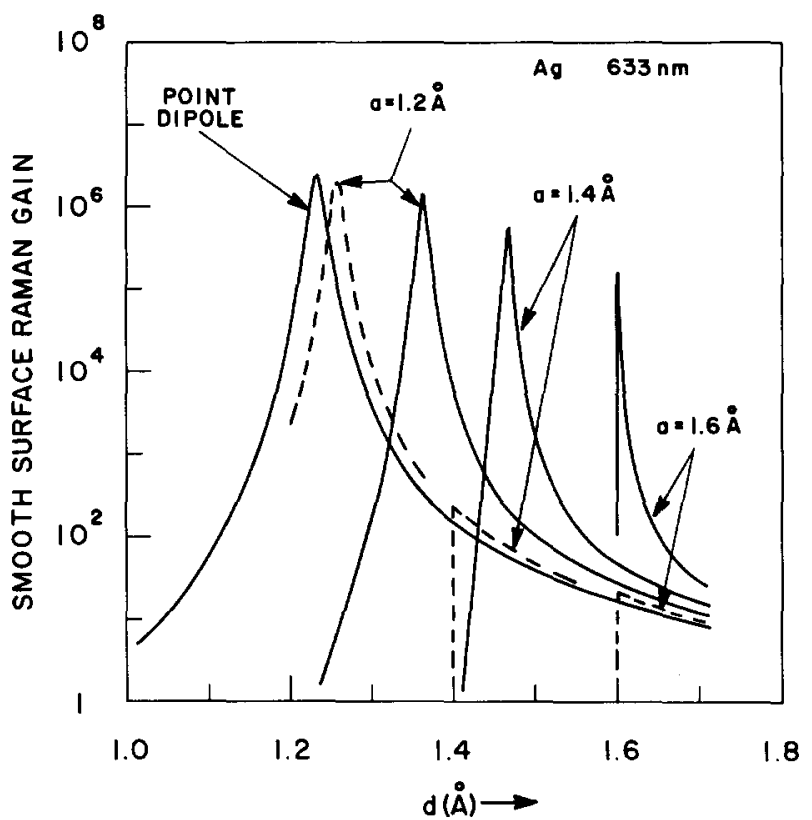

Fig. 25. Image enhancement Raman gain calculated using eq. (5.11) for a finite sized molecule modeled as a dielectric sphere (solid lines) or an infinite spherical well (dashed lines). The SCIB model is used and the metal parameters are the same as for fig. 24 . The Raman shift is $1000 \mathrm{~cm}^{-1}$. After ref. [42].

dielectric sphere "molecule", however, shows a dramatic change from point-like behavior. The position of maximum gain is pushed out and occurs just outside the sphere radius, which means the sphere is nearly touching the surface. The peak gain is reduced and the gain curve is substantially narrowed. It is significant that the peak occurs when the molecule is nearly resting on the surface, suggesting that the precise placement is not a problem for real molecules.

An additional effect that now must also be considered is the motion of the molecule in the adsorption potential. We considered this effect in chapter 4, where expression (4.73) gives the amplitude of the motion. Typical measured vibrational frequencies for this motion are $\omega_{\mathrm{a}}=10^{2} \mathrm{~cm}^{-1}$, and, choosing a mass $M=10^{2} \mathrm{amu}$, the rms amplitude of the motion at room temperature should be $\langle\Delta z\rangle^{1 / 2} \cong 0.08 \AA$. This motion, being larger than the widths of the gain curves in figs. 24 and 25, relieves somewhat the burden of precise placement of the molecule in the point-dipole calculations. It also means the gain will be reduced. For gain curves such as those in fig. 25 using spheres of radius $a=1.6-1.8 \AA$ (reasonable values for pyridine), this amounts to multiplying the peak gain by the ratio of the width of the curve to the width of the zero-point motion $\left(2\left\langle\Delta z^{2}\right\rangle^{1 / 2}\right)$. The resulting SERS gain is $\sim 10^{3}$. Clearly this is an order of magnitude estimate. Even so it is much too small to explain the enhancements of $10^{5}-10^{6}$ observed for pyridine on Ag. We can conclude that there must be additional effects contributing to the SERS gains on $\mathrm{Ag}$, even though image enhancement may contribute significantly.

The discussion so far has concentrated on $\mathrm{Ag}$, which is the most favorable metal with regard to image enhancement. Other noble metals and the alkali metals generally have larger electron scattering rates $\nu$, and most transition metals have large interband contributions to $\operatorname{Im}\left(\varepsilon_{2}\right)$. In either case a decrease in the image enhancement gain is expected. Liquid $\mathrm{Hg}$ is an interesting case, since it has been reported to show SERS gains of $10^{4}-10^{6}$ [100], and, like $\mathrm{Ag}$, it has a significant core polarization term $\varepsilon_{\mathrm{b}}$ and no interband absorption [101]. The primary difference is that the electron scattering rate is about 20 times 
larger in liquid $\mathrm{Hg}$. Figure 26 shows calculated results for pyridine on liquid $\mathrm{Hg}$ obtained from the nonlocal SCIB model for both a point dipole and a dielectric sphere [102]. The dashed lines for Ag, shown for comparison, are obtained using the same parameters as those for fig. 25, excepting $\hbar \omega_{0}=2.41 \mathrm{eV}$. These calculations predict an image enhancement gain for liquid $\mathrm{Hg}$ of about 10 . Moreover, there are no roughness effects of the sort to be discussed later in this chapter that can significantly increase the Raman signal from a molecule on liquid $\mathrm{Hg}$. It is thus a reasonable conclusion that SERS gains of $10^{4}-10^{6}$ on liquid $\mathrm{Hg}$ are not consistent with an electromagnetic explanation.

Eesley and Smith [103] have pointed out the possibility of an additional image factor that should be introduced when the internal vibrational mode of the molecule is infrared active as well as Raman active. In this case the zero-point fluctuations of the vibration produce an image field interacting with it at the vibrational frequency $\omega_{\mathrm{v}}$. The result is an additional factor in eq. (5.7), which is like the other two but is evaluated at $\omega_{\mathrm{v}}$. One problem with this new factor, as we have shown in the previous chapter, is that, if there is a resonant enhancement between the fluctuating dipole and its image, then there will be an associated large frequency shift. Since observed frequency shifts of adsorbed molecules are typically only a few percent, we do not expect this additional image enhancement factor to be important in most cases.

Hilton and Oxtoby [104] have included finite molecular size in a quantum mechanical calculation of the image effect. They do this by means of a Hartree calculation applied to a hydrogen atom together with its image obtained by reflecting the wavefunctions in the surface. The maximum calculated increase in the effective static polarizability $\alpha_{\text {eff }}$ was quite small (about a factor of three), and it occurred closer to the surface than it would have for the simple point-dipole model. From these results they concluded that image enhancement was not important in SERS. However, even a factor of 3 in $\alpha_{\text {eff }}$ gives a SERS

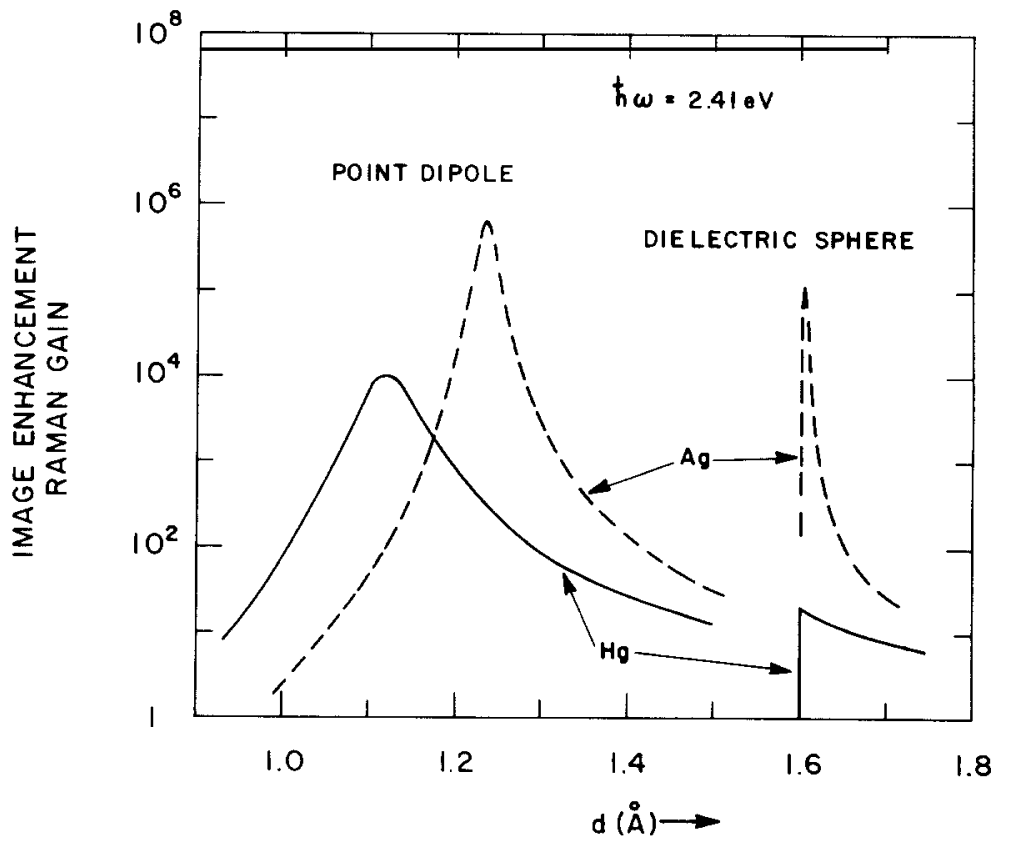

Fig. 26. Image enhancement Raman gain calculated using the SCIB model for liquid $\mathrm{Hg}$ (solid curves). The parameters are $\hbar \omega_{\mathrm{p}}=10.6 \mathrm{eV}$, $k_{\mathrm{F}}=1.34 \times 10^{8} \mathrm{~cm}^{-1}, v_{\mathrm{F}}=1.55 \times 10^{8} \mathrm{~cm} / \mathrm{s}, \varepsilon_{\mathrm{b}}=2, h \nu=1.37 \mathrm{eV}, \alpha_{1}=11.3 \AA^{3}$, and a $1000 \mathrm{~cm}^{-1}$ Raman shift. The dashed curves correspond to Ag. The curves on the right are for a $1.6 \AA$ radius dielectric sphere molecular model. After ref. [102] 
gain of $10^{2}$, which is significant. It is clear from their calculations that a quantum mechanical model of the molecule will lead to a reduction in, but not necessarily an elimination of, the image enhancement gain.

We would like to conclude this section with a definite answer as to whether or not image enhancement is important in SERS. That image enhancement exists is not in dispute. Maxwell's equations demand it. The real question is whether or not it gives a large effect for realistic spacings of the molecule from the metal. In the most favorable case, the SCIB model applied to Ag with a core polarization term, the estimated magnitude of the effect is $10^{3}$. This value will depend only weakly on the frequency, since there is an automatic positioning of the molecule at the distance for maximum gain. Free electron models that neglect core polarization do not generally give large SERS gains at reasonable distances. On the other hand, in our discussion we have taken the molecular polarizability $\alpha_{1}$ to be a real, frequency-independent constant. This is a good assumption as long as the pump frequency $\omega_{0}$ is small compared with the frequency $\omega_{\mathrm{e}}$ of the first electronic excited state of the molecule. If this is not the case then we have the possibility of resonant Raman scattering, and we must take $\alpha_{1}$ to be of the form

$$
\alpha_{1}(\omega)=\alpha_{1}(\infty)+\left[\alpha_{1}(0)-\alpha_{1}(\infty)\right] \omega_{\mathrm{e}}^{2} /\left(\omega_{\mathrm{e}}^{2}-\omega^{2}-\mathrm{i} \omega \Gamma\right)
$$

In this case, when $\Gamma$ is small, we expect large image enhancements at reasonable spacings when $\omega_{0}$ is in the narrow frequency range around $\omega_{\mathrm{e}}$. This will be true for any of the models of the metal response described in chapter 2, with or without core polarization. The difficulty with ascribing SERS gains to resonance Raman of this sort is that there is in most experiments no indication of a narrow resonance in the SERS excitation profile, nor does the free molecule show any electronic absorption at the pump frequency for which the SERS gain is greatest. We can conclude that, while there is no convincing experimental evidence either for or against the image enhancement effect, it may in certain cases make a significant contribution to the SERS gain.

In the following sections we discuss electromagnetic enhancement effects that arise from macroscopic modifications to the shape of the surface, such as random roughening or gratings being ruled onto the surface. The scale for such modifications is from many tens to several hundred angstroms, i.e., much greater than atomic dimensions but also much smaller than the wavelength of light. The image effects described in this section involve the shape of the metal surface on an atomic scale, $1-5 \AA$, and thus will be unchanged. This means the factors associated with image enhancement will multiply whatever subsequent gain factors that result from structural surface changes. The major difference between the image and the roughness effects is the range of the interaction. As is clear from figs. 24 and 25 , the range of image enhancement is at most a few angstroms. In contrast, the range of roughness effects is $10-10^{2} \AA$.

\subsection{SERS from metal gratings}

We next consider the effects on SERS occurring when the metal is roughened in a very special way, namely, by the ruling, usually by photolithographic techniques, of a periodic grating onto an otherwise smooth surface. There have been several experimental investigations of SERS from metal grating surfaces [105-107] and numerous theoretical discussions of the problem as well [108-111]. The primary effect can be associated with the resonant excitation of propagating surface plasmons on the metal surface through first-order coupling of the incident beam with the grating. The SERS signal is observed 
to increase dramatically when the condition for surface plasmon excitation is satisfied,

$$
|\boldsymbol{p}+\boldsymbol{g}|=p_{\mathrm{SP}},
$$

where $\boldsymbol{p}$ is the projection of the incident wavevector onto the surface, $\boldsymbol{g}$ is a reciprocal lattice vector of the grating, and $p_{\mathrm{SP}}$ is the surface plasmon wavevector given by (3.19). The vector $\boldsymbol{g}$ is in the surface plane, perpendicular to the grooves, and its magnitude is a multiple of $2 \pi / D$, where $D$ is the groove spacing.

The increased SERS signal comes about because the optical electric field in the surface plasmon mode can be much larger than that in the incident beam. When condition (5.13) is satisfied there is also a narrow dip in the reflectivity of the specular beam. The situation is completely analogous to the attenuated-total-reflection (ATR) dip observed in prism excitation of surface plasmons [112]. An increased SERS signal has also been observed for the case of prism excited surface plasmons [113]. Our aim is to calculate the field enhancement. For prism excitation the field enhancement is given by a simple application of the multilayer Fresnel formulas [5]. For grating excitation exact calculations of the field enhancement are much more difficult and require a knowledge of the depth and profile of the grooves [114]. Below we outline an alternative method based on energy conservation that is useful in most of the interesting cases, i.e., those in which the enhancement is large [115].

Consider a P-polarized optical beam incident on a metal surface as shown in fig. 27 , such that the vectors $\boldsymbol{k}, \boldsymbol{p}$, and $\boldsymbol{g}$ lie in the $x z$ plane, and assume that the resonance condition is satisfied resulting in a surface plasmon propagating in the $x$ direction. Since we are interested in cases with large enhancement, we assume an optimized grating on a high reflectivity metal. This requires that the radiation damping induced by the grating matches the intrinsic damping of the surface plasmon mode. Since, by assumption, the intrinsic damping is small, then the optimized grating will represent only a small perturbation to the smooth surface, and we can represent the fields for the mode with those appropriate for a perfectly smooth surface. The only place this assumption breaks down is at the zone boundaries $\left(p_{\mathrm{SP}} \cong g\right)$ of the reciprocal lattice space of the grating, where tiny band gaps in the $\omega$ vs. $p_{\mathrm{SP}}$ curve are introduced [116].

The surface plasmons are P-polarized waves associated, as we saw in section 3.2, with the vanishing of the denominator in the Fresnel formulas (2.6). When the denominator vanishes, the ratio of $t_{12}^{\mathrm{P}}$ to $r_{12}^{\mathrm{P}}$ is unity. Therefore, the form of the fields in the surface plasmon mode is obtained from the general

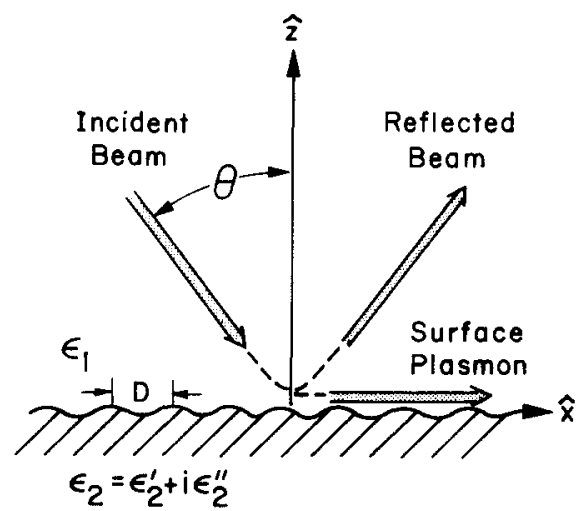

Fig. 27. Schematic diagram showing the excitation of a surface plasmon wave via a grating on a metal. 
form (2.9) and (2.10) by dropping the incident wave and setting $r_{12}^{\mathrm{P}}=t_{12}^{\mathrm{P}}=1$, i.e., absorbing $r_{12}^{\mathrm{p}}$ into the arbitrary amplitude $B_{\mathrm{p}}$. The fields in the mode are thus given by

$$
\begin{aligned}
& \boldsymbol{B}_{\mathrm{SP}}=B_{y} \hat{\boldsymbol{y}} \exp \left\{\mathrm{i}\left(p_{\mathrm{SP}} x-\omega t\right)\right\} \begin{cases}\exp \left(\mathrm{i} q_{1} z\right), & z>0, \\
\exp \left(-\mathrm{i} q_{2} z\right), & z<0,\end{cases} \\
& \boldsymbol{E}_{\mathrm{SP}}=\frac{c}{\omega} B_{y} \exp \left\{\mathrm{i}\left(p_{\mathrm{SP}} x-\omega t\right)\right\} \begin{cases}\left(q_{1} \hat{\boldsymbol{x}}-p_{\mathrm{SP}} \hat{z}\right) \exp \left(\mathrm{i} q_{1} z\right) / \varepsilon_{1}, & z>0, \\
\left(-q_{2} \hat{\boldsymbol{x}}-p_{\mathrm{SP}} \hat{z}\right) \exp \left(-\mathrm{i} q_{2} z\right) / \varepsilon_{2}, & z<0,\end{cases}
\end{aligned}
$$

where the $q_{j}$ are given by (2.8) and $p_{\mathrm{SP}}$ by (3.19). The time averaged power flow in the mode in the $x$ direction per unit length in the $y$ direction is given by

$$
\mathscr{P}_{\mathrm{SP}}=\frac{c}{8 \pi} \int_{-\infty}^{\infty} \mathrm{d} z \operatorname{Re}\left\{\boldsymbol{E}_{\mathrm{SP}} \times \boldsymbol{B}_{\mathrm{SP}}^{*}\right\} \cdot \hat{\boldsymbol{x}}
$$

which using (5.14) and (5.15) can be written

$$
\mathscr{P}_{\mathrm{SP}}=\frac{\omega \varepsilon_{1}^{2}}{16 \pi} \frac{\left|E_{\mathrm{SP}}\left(0^{+}\right)\right|^{2}}{\left|q_{1}\right|^{2}+\left|p_{\mathrm{SP}}\right|^{2}} \operatorname{Re}\left(p_{\mathrm{SP}} / \varepsilon_{1} q_{1}^{\prime \prime}+p_{\mathrm{SP}} / \varepsilon_{2} q_{2}^{\prime \prime}\right)
$$

where $\boldsymbol{E}_{\mathrm{SP}}\left(0^{+}\right)$is the electric vector just outside the metal surface and $q_{j}^{\prime \prime}$ is the imaginary part of $q_{j}$. (For brevity in the remainder of this chapter the real and imaginary parts of a complex quantity will be denoted by prime and double prime, respectively.) The power per unit area dissipated in the metal by the mode is given by

$$
-\mathrm{d} \mathscr{P}_{\mathrm{SP}} / \mathrm{d} x=\alpha \mathscr{P}_{\mathrm{SP}}
$$

where $\alpha=2 p_{\mathrm{SP}}^{\prime \prime}$ is the power attenuation coefficient of the mode. The net power added to the metal per unit area by the external beam is given by the difference in intensities of the incident and reflected beams:

$$
I_{0}-I_{\mathrm{R}}=c \varepsilon_{1}^{1 / 2} \cos \theta\left|E_{0}\right|^{2}(1-R) / 8 \pi
$$

where $\boldsymbol{E}_{0}$ is the electric vector in the incident beam and $R$ is the power reflectivity. Under steady-state conditions the right-hand sides of eqs. (5.19) and (5.18) must be equal, and using (5.17) we obtain the following expression for the field enhancement,

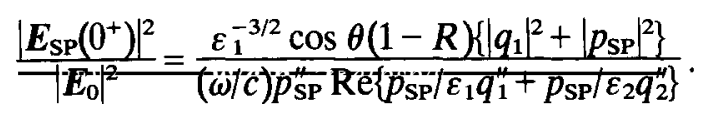

We now make use of the assumption that we are dealing with a good metal for which $\varepsilon_{2}^{\prime \prime} \ll-\varepsilon_{2}^{\prime}$ and $-\varepsilon_{2}^{\prime}>\varepsilon_{1}$. This allows us to express the result in terms of the dielectric constants,

$$
\left|\boldsymbol{E}_{\mathrm{SP}}\left(0^{+}\right)\right|^{2} /\left|\boldsymbol{E}_{0}\right|^{2}=2 \cos \theta \varepsilon_{2}^{\prime 2}(1-R) / \varepsilon_{1}^{1 / 2} \varepsilon_{2}^{\prime \prime}\left(-\varepsilon_{2}^{\prime}-\varepsilon_{1}\right)^{1 / 2} \text {. }
$$


A similar equation, which is nearly the same numerically, has also been derived on the basis of energy conservation for a prism coupler [117].

With a truly optimum grating zero reflectivity can be achieved when the reradiated beam from the surface plasmon exactly cancels the specular reflection. We can thus set $R=0$ in (5.21) to obtain an upper limit on the field enhancement. Calculated results for this upper limit are shown in fig. 28, where we have used a grating spacing of $800 \mathrm{~nm}$ and assumed first order coupling from the grating, i.e., $\sin \theta=\left\{\varepsilon_{2}^{\prime} /\left(1+\varepsilon_{2}^{\prime}\right)\right\}^{1 / 2}-\lambda / d$. The lines in this figure are drawn through points calculated from optical data appropriate for smooth films exposed to air [81].

The large electric field enhancement represented by (5.21) arises from a simple focusing effect. A plane wave that is extended in two directions is compressed into a surface wave extended in only one direction. The maximum increase in intensity in the surface wave is given approximately by the ratio of the distance along the surface that contributes coherently to the wave, which is $\left(2 p_{\mathrm{SP}}^{\prime \prime}\right)^{-1}$, to the spread of the wave in the $z$ direction, which is $\left(2 q_{1}^{\prime \prime}\right)^{-1}$, neglecting the field in the metal. This gives the approximate formula

$$
\left|\boldsymbol{E}_{\mathrm{SP}}\left(0^{+}\right)\right|^{2} /\left|\boldsymbol{E}_{0}\right|^{2} \cong q_{1}^{\prime \prime} / p_{\mathrm{SP}}^{\prime \prime} \cong-2\left(-\varepsilon_{2}^{\prime}\right)^{1 / 2}\left(\varepsilon_{2}^{\prime}-\varepsilon_{1}\right) / \varepsilon_{1}^{1 / 2} \varepsilon_{2}^{\prime \prime},
$$

which is valid whenever $-\varepsilon_{2}^{\prime} \gg \varepsilon_{1}$, in which case it yields results differing only slightly from eq. (5.21).

Equation (5.21) with $R=0$ provides an upper limit to the field enhancement for a perfect grating, i.e., $100 \%$ coupling efficiency. It can also be used to estimate the actual field enhancement for less efficient coupling in cases for which only one diffracted beam needs to be considered. Yamashita and Tsuji [108] have shown for this case, through detailed calculations and comparisons with the experiments of Hutley and Maystre [118], that the formula (5.21) gives accurately their calculated values for the field enhancement. This means that the enhancement in a particular experiment can be determined using (5.21) with the measured $R$ value, and it is not necessary to know the depth or profile of the grooves, quantities that must be known in order to use the more detailed calculations.

The grating can also influence the radiation by the molecule at the Raman shifted frequency. The primary effect here is that radiation emitted by the dipole into surface plasmons will be coupled out by

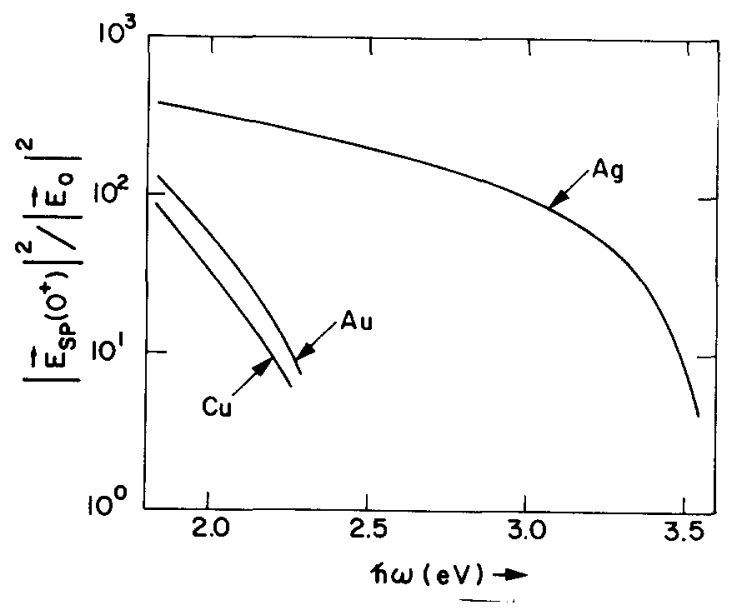

Fig. 28. Maximum field enhancement squared $(R=0)$ calculated from eq. (5.21) for a grating with an $800 \mathrm{~nm}$ spacing on $\mathrm{Ag}$, $\mathrm{Au}$, and $\mathrm{Cu}$ surfaces. After ref. [115]. 
the grating. This will lead to a net increase in the total SERS signal, which can be estimated from plots such as those in fig. 6 . There we see that the power emitted into surface plasmons exceeds that emitted into photons by only a factor of $2-3$, and only for a $\perp$ dipole. This means that the grating-induced gain associated with the emission is at most a factor of $2-3$. On the other hand the emission will be concentrated in very narrow angular ranges, as determined by the condition (5.13), within which the power emitted per steradian can be as much as $\sim 10^{2}$ greater than it would be without the grating.

We have shown in this section that a grating on a high-reflectivity metal such as $\mathrm{Ag}$ can produce a maximum SERS increase of several hundred. The effect is long range (the molecules can be several hundred angstroms from the surface), it is not molecular specific, and it is primarily a one-way effect, i.e., only the incident field is enhanced, not the emitted field. Grating enhancements of the magnitude indicated here have been observed experimentally $[105,106]$, but they are far below the $10^{5}-10^{6}$ SERS gains often seen on randomly roughened surfaces for which, as we will show in the following section, no significant enhancements due to the excitation of propagating surface plasmons can be expected.

\subsection{Random roughness}

We now consider a randomly roughened metal surface, but we specifically exclude the possibility of resonant microstructures. The resonance effect is discussed in the next section. The type of roughness we have in mind here is random bumps of height $50-100 \AA$ with a spacing between bumps comparable to the bump height. Since the translational symmetry of the surface has been broken by the roughness, there will be Fourier components of the surface profile for which the condition (5.13) is satisfied. This means that propagating surface plasmons will be excited by the incident beam in much the same manner as by a grating. It has been suggested that this may contribute to the SERS gain [119-121].

For the purpose of assessing the importance of the surface-plasmon excitation process for SERS we can use the results of the last section. We simply replace the quantity $1-R$ in eq. (5.21) with the roughness-induced power absorbance $A$ of the surface. The resulting equation gives again an upper limit on the field enhancement, since it assumes a coherent scattering into surface plasmons over a length $\alpha^{-1}$, and it does not allow for an increased $\varepsilon_{2}^{\prime \prime}$ produced by the roughness. The quantity $A$ has been measured for $\mathrm{Ag}$ film roughened by overgrowing on a $\mathrm{CaF}_{2}$ layer $[122,123]$ and for $\mathrm{Ag}$ electrodes roughened by electrochemical cycling in a pyridine solution [124]. Both types of roughening are known to produce SERS. In either case $A$ is on the order of a few percent in the $2-3 \mathrm{eV}$ region, which implies a maximum field enhancement of order unity. We can conclude, therefore, that random roughness does not lead to a significant SERS gain through the process of exciting propagating surface plasmons.

Next we consider the effect random roughness has on the emitted radiation. A general discussion of theoretical methods for dealing with random roughness has been given by Maradudin [114]. The method we use has been outlined by us in an earlier publication [43], and it is similar to that described by Kröger and Kretschmann [125] and others [114, 126]. The idea is that the electric fields of the dipole oscillating at the Raman frequency $\omega_{R}$ drive currents in the nearby roughness features, and we calculate the net radiation produced. The roughness induced currents can be represented in the form

$$
j(r)=-\mathrm{i} \omega_{\mathrm{R}} h(\rho) E(\rho) \delta(z),
$$

where $\boldsymbol{E}(\boldsymbol{\rho})$ is the electric field produced by the molecular dipole and $h(\boldsymbol{\rho})$, which has the dimensions of a length, is the polarizability per unit area of the surface. The quantity $h(\boldsymbol{\rho})$ is a statistical quantity that 
can be characterized by an ensemble average. We can think of the function $h(\boldsymbol{\rho})$ as assigning to each bump on the surface a polarizability characteristic of the shape and size of the bump. For example, in the limit of discrete roughness features $h(\boldsymbol{\rho})$ could be approximated as a sum of delta functions

$$
h(\boldsymbol{\rho})=\sum_{j} \alpha_{j} \delta\left(\boldsymbol{\rho}-\boldsymbol{\rho}_{j}\right)
$$

where $\alpha_{j}$ is the polarizability of the feature at $\boldsymbol{\rho}_{j}$. In this case $\alpha_{j}$ and $\boldsymbol{\rho}_{j}$ would be considered as random variables. For protrusions with an aspect ratio of order unity, $h(\boldsymbol{\rho})$ approximately describes the height of their deviations from the mean surface.

To estimate the magnitude of the radiation produced by the current (5.23) we assume the molecular dipole is oriented $\perp$ to the mean surface $\mu\left(\omega_{R}\right)=\mu_{\perp}\left(\omega_{R}\right) \hat{z}$ and take a Gaussian distribution for $h(\boldsymbol{\rho})$,

$$
\langle h(\boldsymbol{\rho})\rangle=0 \quad \text { and } \quad\left\langle h(\boldsymbol{\rho}) h\left(\boldsymbol{\rho}^{\prime}\right)\right\rangle=\left\langle h^{2}\right\rangle \exp \left(-\left|\boldsymbol{\rho}-\boldsymbol{\rho}^{\prime}\right|^{2} / \sigma^{2}\right) \text {. }
$$

In these equations $\langle\cdots\rangle$ represents an ensemble average. We further assume that the molecule is very close to the surface, which means that the near fields of the dipole dominate in exciting the current (5.23). This also means that for calculating the long-wavelength emission into photons and surface plasmons the current (5.23) is equivalent to a point-like dipole $\boldsymbol{\mu}_{\text {rough }}$ given by

$$
\mu_{\text {rough }}=\int \mathrm{d} \rho h(\rho) E_{z}(\boldsymbol{\rho}) \hat{z}
$$

The radiated power can now be calculated from the general formula (3.15) for the power radiated by a dipole near the surface. Taking $\mu_{\|}=0$ and $\mu_{\perp}=\boldsymbol{\mu}_{\text {rough }} \cdot \hat{z}$, we find

$$
\mathscr{P}_{\text {rough }}=\frac{\omega_{\mathrm{R}}}{2 \varepsilon_{1}}\left\langle\boldsymbol{\mu}_{\text {rough }}^{2}\right\rangle \operatorname{Re} \int_{0}^{p_{\max }} \mathrm{d} p \frac{p^{3}}{q_{1}}\left(1+r_{12}^{\mathrm{P}} \exp \left\{2 \mathrm{i} q_{1} d\right\}\right) .
$$

In the integral over $p$ we take $p_{\max }$ to be slightly above $p_{\mathrm{SP}}$, the surface plasmon wavevector (3.19). The assumption here is that emitted surface plasmons will ultimately be scattered into photons via the surface roughness and thus are equivalent to the direct emission of a photon.

The quantity that we must evaluate is

$$
\left\langle\boldsymbol{\mu}_{\text {rough }}^{2}\right\rangle=\int \mathrm{d} \boldsymbol{\rho} \mathrm{d} \boldsymbol{\rho}^{\prime}\left\langle h(\boldsymbol{\rho}) h\left(\boldsymbol{\rho}^{\prime}\right)\right\rangle E_{z}^{*}(\boldsymbol{\rho}) E_{z}\left(\boldsymbol{\rho}^{\prime}\right)
$$

Since $\boldsymbol{\mu}_{\text {rough }}$ is concentrated in the near zone of the molecular dipole, we can use the quasistatic limit of (3.14) for the electric fields

$$
E_{z}(\boldsymbol{\rho})=\frac{\mu}{2 \pi \varepsilon_{1}} \int \mathrm{d} p p \exp (\mathrm{i} \boldsymbol{p} \cdot \boldsymbol{\rho}-p d)\left\{1+\boldsymbol{r}_{12}(p)\right\}
$$

where here and in the following we have dropped the superscript $\mathrm{P}$ from $r_{12}$, it being understood that $r_{12}$ 
is the P-polarized reflection coefficient. We can now write (5.27) in the form

$$
\begin{aligned}
\left\langle\boldsymbol{\mu}_{\text {rough }}^{2}\right\rangle= & \frac{\mu^{2}}{4 \pi^{2} e^{2}} \int \mathrm{d} \boldsymbol{\rho} \mathrm{d} \boldsymbol{\rho}^{\prime} \mathrm{d} \boldsymbol{p} \mathrm{d} \boldsymbol{p}^{\prime}\left\langle h(\boldsymbol{\rho}) h\left(\boldsymbol{\rho}^{\prime}\right)\right\rangle p \exp (-\mathrm{i} \boldsymbol{p} \cdot \boldsymbol{\rho}-p d)\left\{1+r_{12}^{*}(p)\right\} \\
& \times p^{\prime} \exp \left(\mathrm{i}^{\prime} \cdot \boldsymbol{\rho}^{\prime}-p^{\prime} d\right)\left\{1+r_{12}\left(p^{\prime}\right)\right\}
\end{aligned}
$$

To evaluate some of these integrals introduce $\boldsymbol{R}=\left(\boldsymbol{\rho}+\boldsymbol{\rho}^{\prime}\right) / 2, \boldsymbol{r}=\boldsymbol{\rho}-\boldsymbol{\rho}^{\prime}$, and use (5.24),

$$
\begin{aligned}
\left\langle\boldsymbol{\mu}_{\text {rough }}^{2}\right\rangle= & \frac{\mu^{2}\left\langle h^{2}\right\rangle}{4 \pi^{2} \varepsilon_{1}^{2}} \int \mathrm{d} \boldsymbol{R} \mathrm{d} \boldsymbol{r} \mathrm{d} \boldsymbol{p} \mathrm{d} \boldsymbol{p}^{\prime} \exp \left[-\boldsymbol{r}^{2} / \sigma^{2}-\mathrm{i} \boldsymbol{R} \cdot\left(\boldsymbol{p}-\boldsymbol{p}^{\prime}\right)\right. \\
& \left.-\mathrm{i} \boldsymbol{r} \cdot\left(\boldsymbol{p}+\boldsymbol{p}^{\prime}\right) / 2-\left(p+p^{\prime}\right) d\right] p p^{\prime}\left\{1+\boldsymbol{r}_{12}^{*}(p)\right\}\left\{1+\boldsymbol{r}_{12}\left(p^{\prime}\right)\right\} .
\end{aligned}
$$

The integral over $\boldsymbol{R}$ yields $4 \pi^{2} \delta\left(p-p^{\prime}\right)$, therefore (5.30) reduces to

$$
\left\langle\boldsymbol{\mu}_{\text {rough }}^{2}\right\rangle=\frac{\mu^{2}\left\langle h^{2}\right\rangle}{\varepsilon_{1}^{2}} \int \mathrm{d} \boldsymbol{r} \mathrm{d} \boldsymbol{\operatorname { e x p }}\left[-\boldsymbol{r}^{2} / \sigma^{2}-\mathrm{i} \boldsymbol{r} \cdot \boldsymbol{p}-2 p d\right] p^{2}\left|1+r_{12}\right|^{2} .
$$

Performing the integration over $r$ and completing the angular part of the $p$ integration we have

$$
\left\langle\boldsymbol{\mu}_{\text {rough }}^{2}\right\rangle=\frac{2 \pi^{2} \sigma^{2} \mu^{2}\left\langle h^{2}\right\rangle}{\varepsilon_{1}^{2}} \int \mathrm{d} p p^{3}\left|1+r_{12}\right|^{2} \exp \left(-2 p d-\sigma^{2} p^{2} / 4\right)
$$

Without an explicit and simple form for $r_{12}$ the remaining integral cannot be evaluated in closed form. However, if $r_{12}(p)$ is slowly varying, then one can perform the integral approximately by evaluating the factor $\left|1+r_{12}(p)\right|^{2}$ at the position where the remaining factors in the integrand peak. In the case $d \ll \sigma$, this peak occurs at $p=\sqrt{6} / \sigma$, and we obtain

$$
\left\langle\mu_{\text {rough }}^{2}\right\rangle=\left(16 \pi^{2} \mu^{2}\left\langle h^{2}\right\rangle / \varepsilon_{1}^{2} \sigma^{2}\right)\left|1+r_{12}(p=\sqrt{6} / \sigma)\right|^{2} .
$$

For the roughness scale we have in mind, $\sigma \sim 50 \AA$, this means $p \sim 0.05 \AA^{-1}$ or $p / 2 k_{\mathrm{F}} \sim 0.02$. Referring to fig. 3 we see that for $p$ values in this range we can take $r_{12}=1$ with little loss of accuracy giving

$$
\left\langle\mu_{\text {rough }}^{2}\right\rangle=64 \pi^{2}\left\langle h^{2}\right\rangle \mu^{2} / \varepsilon_{1}^{2} \sigma^{2} .
$$

The total scattered power produced by the roughness is now given by (5.26), which we write in the form

$$
\mathscr{P}_{\text {rough }}=\frac{64 \pi^{2}\left\langle h^{2}\right\rangle}{\epsilon_{1}^{2}}\left[\frac{\omega_{\mathrm{R}} \mu^{2}}{2 \epsilon_{1}} \operatorname{Re} \int_{0}^{p_{\max }} \mathrm{d} p p^{3}\left(1+r_{12} \exp \left(2 \mathrm{i} q_{1} d\right)\right)\right]
$$

The quantity in the square brackets is the sum of $\mathscr{P}_{\text {photon }}$ (3.18), which is the signal from a smooth surface, and $\mathscr{P}_{\text {SP }}$ (3.21). We can therefore write $\mathscr{P}_{\text {rough }}$ as a gain factor $G_{\text {rough }}$ multiplying the smooth surface signal, $\mathscr{P}_{\text {rough }}=G_{\text {rough }} \mathscr{P}_{\text {photon }}$ where 


$$
G_{\text {rough }}=\frac{64 \pi^{2}\left\langle h^{2}\right\rangle}{\varepsilon_{1}^{2} \sigma^{2}}\left(1+\mathscr{P}_{\mathrm{SP}} / \mathscr{P}_{\text {photon }}\right)
$$

The fact that the ratio $\left\langle h^{2}\right\rangle / \sigma^{2}$ occurs in (5.36) has a simple physical interpretation that could have been anticipated on intuitive grounds. It tells us that the roughness gain is proportional to the scattering strength of the typical roughness feature, which is $\left\langle h^{2}\right\rangle$, multiplied by the surface density of such features, which is $\sigma^{-2}$.

To estimate the magnitude of $G_{\text {rough }}$ we need a model of the surface topography that allows us to estimate $\left\langle h^{2}\right\rangle / \sigma^{2}$. This is accomplished by representing the roughness features themselves as polarizable ellipsoids for which the polarizability along the $j$ th axis is [4]

$$
\alpha_{j}=\frac{1}{3} a b c \varepsilon_{1}\left(\varepsilon_{2}-\varepsilon_{1}\right) /\left[\varepsilon_{1}+L_{j}\left(\varepsilon_{2}-\varepsilon_{1}\right)\right],
$$

where $a, b$, and $c$ are the semi-axes of the ellipsoid and $L_{j}$ is the depolarization factor. We take $\alpha_{j}$ to be along the $c$ axis, which is normal to the surface, and drop the $j$ subscript. The "area" per ellipsoid is then $\pi a b$, and $\left\langle h^{2}\right\rangle^{1 / 2}$ is given approximately by

$$
\left\langle h^{2}\right\rangle^{1 / 2}=\frac{c \varepsilon_{1}}{3 \pi} \frac{\overline{\varepsilon_{2}-\varepsilon_{1}}}{\varepsilon_{1}+L\left(\varepsilon_{2}-\varepsilon_{1}\right)},
$$

and using this result $(5.36)$ becomes

$$
G_{\text {rough }}=\frac{64 c^{2}}{9 \sigma^{2}}\left|\frac{\varepsilon_{2}-\varepsilon_{1}}{\varepsilon_{1}+L\left(\varepsilon_{2}-\varepsilon_{1}\right)}\right|^{2}\left(1+\mathscr{P}_{\mathrm{SP}} / \mathscr{P}_{\text {photon }}\right)
$$

For a sphere $L=1 / 3$, for an extremely oblate ellipsoid in the flat direction $L=1$, and for a needle shaped one in the long direction $L=0$.

The roughness of a metal surface is usually characterized by an rms roughness height $\delta$ and a correlation length $\sigma$ associated with the Gaussian correlation [114]. Typical experimental values for a "smooth" evaporated metal film are $\delta=10 \AA$ and $\sigma=100-200 \AA$. This sort of roughness can be modeled by taking $L=1, c=10 \AA$, and $\sigma=10^{2} \AA$ in (5.39). For a smooth film we also cannot include the term $\mathscr{P}_{\mathrm{SP}} / \mathscr{P}_{\text {photon }}$ in (5.39), since it is known that the intrinsic damping in such films far outweighs the radiation loss of surface plasmons due to roughness scattering [82]. Applying these results to a $\mathrm{Ag}$ film at $\hbar \omega=2 \mathrm{eV}$, we find $G_{\text {rough }}=0.1$. Our model thus predicts very little roughness gain for a smooth evaporated film.

For a highly roughened surface the situation is quite different. We envision the roughness here as a dense packing of boss-like or somewhat prolate protrusions that might be formed during the electrochemical cycling of an electrode or when an evaporated film is grown and maintained at low temperatures. The roughness gain now increases rapidly with increasing aspect ratio $c / a$. This is shown in fig. 29 where we plot $G_{\text {rough }} \sigma^{2} / c^{2}$ from eq. (5.39) as a function of $c / a$. For these calculations we have assumed a prolate spheroidal protrusion $(a=b)$ for which the depolarizing factor is given by [4]

$$
L=\frac{1-e^{2}}{2 e^{3}}\left\{\ln \left(\frac{1+e}{1-e}\right)-2 e\right\}
$$




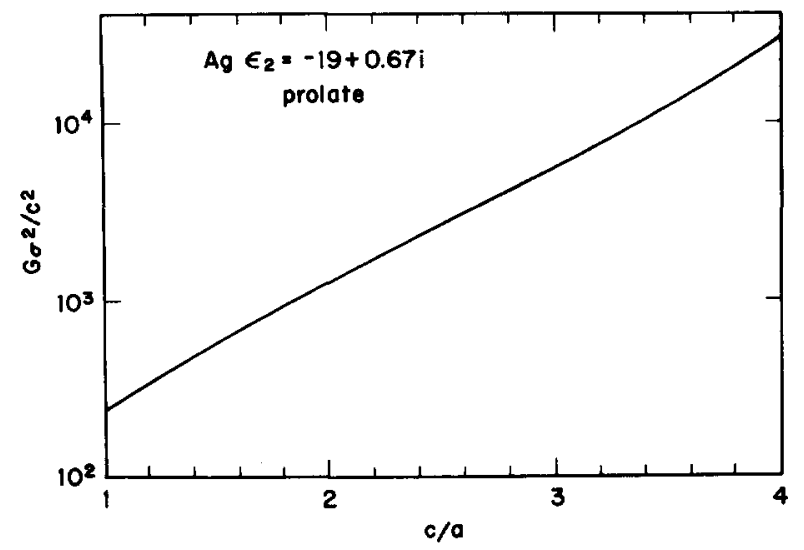

Fig. 29. Normalized roughness gain calculated from eq. (5.39) for prolate spheroidal roughness features on $\mathrm{Ag}$ at $\hbar \omega_{\mathrm{R}}=2 \mathrm{eV}, \varepsilon_{2}=-19+0.67 \mathrm{i}$.

where the eccentricity $e=\left(1-a^{2} / c^{2}\right)^{1 / 2}$. The calculated results in the figure are normalized with respect to the ratio $c^{2} / \sigma^{2}$. However, we would expect this ratio to be of order unity for the sort of roughness considered. We can conclude that a roughness gain of 2 to 3 orders of magnitude is reasonable. This roughness gain is a long-range effect in the sense that the molecule does not have to touch the metal. In fact the range is given approximately by the correlation length $\sigma$, which will typically be many tens of angstroms.

It is tempting to extend the calculation shown in fig. 29 to large enough aspect ratios so that the real part of the denominator in (5.37) vanishes. This will certainly produce very large roughness gains. For $\mathrm{Ag}$ at $\hbar \omega=2 \mathrm{eV}$ this would occur at $L=0.05(c / a=5.4)$, at which point $G_{\text {rough }} \sigma^{2} / c^{2}=6 \times 10^{6}$. A roughness gain of this magnitude pushes the present simple model well beyond its region of validity. We have implicitly assumed that the field driving each roughness feature is that from the original molecular dipole, and that the fields from other roughness features can be ignored. If there is a resonance in the polarizability of the protrusions, then this assumption will no longer hold and we must solve selfconsistently for the fields. The result will be a severely limited gain, since the resonance frequency for a given aspect ratio will be shifted to higher frequencies. A calculation that shows this effect for an island film structure is given by Eagen [127].

\subsection{Resonant microstructures, particle arrays and island films}

In this final SERS section we consider the effect of resonant microstructures. Most of the experiments on these structures involve samples composed of photolithographically produced metal particle arrays $[54,55]$ or metal-island films $[51-53,128]$. However, the essential physics that gives rise to large SERS enhancements is quite simple and can be demonstrated by considering a single isolated ellipsoid. The molecule is in the vicinity of a metal particle that has a resonance in its polarizability. The molecule feels an enhanced field at $\omega_{0}$ due to the polarization of the metal particle. In addition, the molecular dipole at $\omega_{\mathbf{R}}$ polarizes the metal particle, greatly enhancing the emitted fields. Since $\omega_{0}$ and $\omega_{\mathbf{R}}$ are nearly the same, there can be a two-way enhancement. The metal particle acts as an antenna that increases both the incident and emitted fields. There have been several previous treatments of this effect $[60-62,129-133]$, therefore our discussion will be brief and will omit many details. 


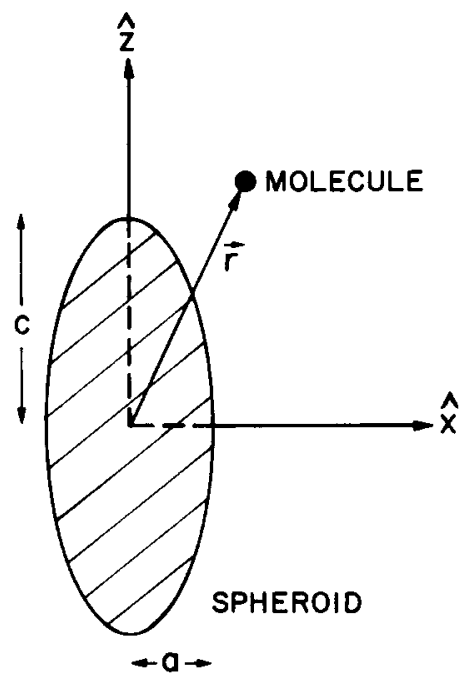

Fig. 30. Geometry for molecule near a spheroidal metal particle.

Consider a molecule a distance $r$ from a polarizable metal particle, as shown in fig. 30 , subject to an applied field $E_{0} \exp \left(-\mathrm{i} \omega_{0} t\right)$. For simplicity we take the particle to be a spheroid with the $c$ axis along $\hat{z}$ and the $a$ and $b$ axes equal. The assumption is that the molecule is some tens of angstroms from the surface of the particle, so that image effects are negligible and also that we need consider only the dipole fields induced in the spheroid. The induced molecular dipole at $\omega_{0}$ can be written

$$
\boldsymbol{\mu}\left(\omega_{0}\right)=\alpha_{1}\left(\boldsymbol{E}_{0}+\left[3 \boldsymbol{\mu}_{\mathrm{s}}\left(\omega_{0}\right) \cdot \hat{\boldsymbol{r}} \hat{\boldsymbol{r}}-\boldsymbol{\mu}_{\mathrm{s}}\left(\omega_{0}\right)\right] / \varepsilon_{1} r^{3}\right),
$$

where $\boldsymbol{\mu}_{\mathrm{s}}\left(\omega_{0}\right)$ is the induced dipole moment in the spheroid given by

$$
\begin{aligned}
\boldsymbol{\mu}_{\mathrm{s}}\left(\omega_{0}\right)= & \alpha_{\mathrm{s}, \|}\left\{\hat{\boldsymbol{z}} \cdot \boldsymbol{E}_{0}+\left[3 \hat{\boldsymbol{z}} \cdot \hat{\boldsymbol{r}} \boldsymbol{\mu}\left(\omega_{0}\right) \cdot \hat{\boldsymbol{r}}-\hat{\boldsymbol{z}} \cdot \boldsymbol{\mu}\left(\omega_{0}\right)\right] / \varepsilon_{1} \boldsymbol{r}^{3}\right\} \hat{\boldsymbol{z}} \\
& +\alpha_{\mathrm{s}, \perp}\left\{\boldsymbol{E}_{0}-\hat{\boldsymbol{z}} \cdot \boldsymbol{E}_{0} \hat{\boldsymbol{z}}+\left[3(\hat{\boldsymbol{r}}-\hat{\boldsymbol{z}} \cdot \hat{\boldsymbol{z}} \hat{\boldsymbol{z}}) \boldsymbol{\mu}\left(\omega_{0}\right) \cdot \hat{\boldsymbol{r}}-\boldsymbol{\mu}\left(\omega_{0}\right)+\hat{\boldsymbol{z}} \cdot \boldsymbol{\mu}\left(\omega_{0}\right) \hat{z}\right] / \varepsilon_{1} \boldsymbol{r}^{3}\right\},
\end{aligned}
$$

in which $\alpha_{\mathrm{s}, \|}$ and $\alpha_{\mathrm{s}, \perp}$ are the $\|$ and $\perp$ components of the spheroid polarizability given by (5.37). We have not included here the image field from the molecular dipole, which would correspond to a term on the right side of (5.41) proportional to $\mu\left(\omega_{0}\right)$. The eqs. (5.41) and (5.42) can be solved for arbitrary orientations of the field and positions of the molecule. However, the primary effect leading to large SERS gains is a resonance in one of the spheroid polarizability components, and it suffices to demonstrate the magnitude of the effect for the simplest geometry. We thus take the applied field along the long axis of the spheroid $\boldsymbol{E}_{0}=E_{0} \hat{z}$ and the molecule situated on this axis above the spheroid. The spheroid polarizability along the $c$ axis is designated simply as $\alpha_{\mathrm{s}}(\omega)$. Solving the above equations for $\mu\left(\omega_{0}\right)$,

$$
\mu\left(\omega_{0}\right)=\alpha_{1}\left\{E_{0}+2 \alpha_{\mathrm{s}}\left(\omega_{0}\right) E_{0} / \varepsilon_{1} r^{3}\right\} \cong 2 \alpha_{1} \alpha_{\mathrm{s}} E_{0} / \varepsilon_{1} r^{3},
$$

where we neglect terms involving $\mu\left(\omega_{0}\right) / \varepsilon_{1} r^{3}$ in (5.42), since there is no resonance in $\alpha_{1}$ and the resonance in $\alpha_{\mathrm{s}}$ is assumed to be sufficiently large that $\alpha_{\mathrm{s}}\left(\omega_{0}\right) / \varepsilon_{1} r^{3} \gg 1$. As in section 5.1 , there will be a 
dipole of magnitude $c_{\mathrm{R}} \mu\left(\omega_{0}\right)$ generated at $\omega_{\mathrm{R}}$ by the internal vibration in the molecule. This dipole will then drive the spheroid at $\omega_{R}$ inducing a dipole moment

$$
\mu_{\mathrm{s}}\left(\omega_{\mathrm{R}}\right)=2 c_{\mathrm{R}} \mu\left(\omega_{0}\right) \alpha_{\mathrm{s}}\left(\omega_{\mathrm{R}}\right) / \varepsilon_{1} r^{3}
$$

The Raman signal is proportional to $\left|\mu_{\mathrm{s}}\left(\omega_{\mathrm{R}}\right)+\mu\left(\omega_{\mathrm{R}}\right)\right|^{2} \cong\left|\mu_{\mathrm{s}}\left(\omega_{\mathrm{R}}\right)\right|^{2}$. In the absence of the spheroid the Raman signal is $c_{\mathrm{R}}^{2} \alpha_{1}^{2} E_{0}^{2}$, therefore the SERS enhancement $G$ is given approximately by

$$
G=16\left|\alpha_{\mathrm{s}}\left(\omega_{0}\right) \alpha_{\mathrm{s}}\left(\omega_{\mathrm{R}}\right)\right|^{2} / \varepsilon_{1}^{4} r^{12} \text {. }
$$

The leading factor of 16 would be 1 if the molecule were chosen to lie on the $x$ axis. Referring to eq. (5.37), the resonance condition is $\varepsilon_{1}+L\left(\varepsilon_{2}^{\prime}-\varepsilon_{1}\right)=0$, at which point $\alpha_{\mathrm{s}}=a^{2} c \varepsilon_{1}\left(\varepsilon_{2}-\varepsilon_{1}\right) / 3 L \varepsilon_{2}^{\prime \prime}$. Typical values for a $3: 1 \mathrm{Ag}$ spheroid $\left(L=0.109, \varepsilon_{2}^{\prime}=-8.2, \hbar \omega_{0}=2.66 \mathrm{eV}, \varepsilon_{2}^{\prime \prime}=0.34\right)$ with $c=300 \AA$ and $r=350 \AA$ yield $G=2 \times 10^{4}$. Corresponding values for a $4: 1$ spheroid $\left(L=0.0754, \varepsilon_{2}^{\prime}=-12.3, \hbar \omega_{0}=\right.$ $2.33 \mathrm{eV}, \varepsilon_{2}^{\prime \prime}=0.5$ ) give $G=1 \times 10^{5}$. The above discussion gives the correct order of magnitude and is generally applicable to very small spheres. In practice, however, two important refinements to the theory are needed, and we address these below.

The first refinement concerns the use of the quasistatic approximation. The expression (5.37) for the polarizability of an ellipsoid is derived using this approximation, the usual justification for which being that the dimensions of the particle are small compared to the wavelength of light. The requirement is actually much more limited. As shown by the calculations of Kerker et al. [61] for spheres and Barber et al. [62] for spheroids, significant deviations from the quasistatic polarizability of a particle occur even when its size is of order $\lambda / 10$, which is the case in most experiments. Wokaun et al. [133] have shown that the first-order corrections produced by the full retarded solutions are associated with radiation damping, and that these can be calculated quite simply. The induced dipole moment in the spheroid produces a radiation reaction field proportional to $\mu_{\mathrm{s}}$, but $90^{\circ}$ out of phase. Representing this field as $E_{\mathrm{r}}=\mathrm{i} \beta \mu_{\mathrm{s}}$, where $\beta$ is a real constant to be determined, the induced moment on the spheroid can be written,

$$
\mu_{\mathrm{s}}\left(\omega_{0}\right)=\alpha_{\mathrm{s}}\left\{E_{0}+\mathrm{i} \beta \mu_{\mathrm{s}}\left(\omega_{0}\right)\right\} \quad \text { or } \quad \mu_{\mathrm{s}}=\alpha_{\mathrm{s}} E_{0} /\left(1-\mathrm{i} \alpha_{\mathrm{s}} \beta\right)
$$

Here $\alpha_{\mathrm{s}}$ is taken to be real. The work done on this dipole by the external field is given by (3.1)

$$
-\frac{1}{2} \omega \operatorname{Im}\left(\mu_{\mathrm{s}}^{*} E_{0}\right)=\frac{1}{2} \omega \alpha_{\mathrm{s}}^{2} \beta E_{0}^{2} /\left(1+\alpha_{\mathrm{s}}^{2} \beta^{2}\right) .
$$

The power radiated by the dipole is given by the Larmor formula (3.11)

$$
\frac{\varepsilon_{1}^{1 / 2} \omega^{4}\left|\mu_{\mathrm{s}}\right|^{2}}{3 c^{3}}=\frac{\varepsilon_{1}^{1 / 2} \omega^{4} \alpha_{\mathrm{s}}^{2} E_{0}^{2}}{3 c^{3} 1+\alpha_{\mathrm{s}}^{2} \beta^{2}}
$$

Equating the right-hand sides of these two equations gives the value of

$$
\beta=\varepsilon_{1}^{1 / 2} 2 \omega^{3} / 3 c^{3} \text {. }
$$

The effective polarizability of the spheroid written as $\alpha_{\mathrm{s}} /\left(1-\mathrm{i} \alpha_{\mathrm{s}} \beta\right)$ now becomes 


$$
\alpha_{\mathrm{eff}}=\frac{\varepsilon_{1} V}{4 \pi} \frac{\varepsilon_{2}-\varepsilon_{1}}{\varepsilon_{1}+\left(\varepsilon_{2}-\varepsilon_{1}\right)\left[L-\mathrm{i} 4 \pi^{2} V / 3 \lambda^{3}\right]}
$$

where $\lambda=2 \pi c / \omega \varepsilon_{1}^{1 / 2}$ is the wavelength of light in medium 1 and $V=4 \pi a^{2} c / 3$ is the volume of the spheroid. This equation represents the first-order correction to (5.37) produced by radiation damping. The correction is implemented by simply replacing the depolarization factor $L$ by $L-i 4 \pi^{2} V / 3 \lambda^{3}$. The new resonance condition is

$$
\varepsilon_{1}+L\left(\varepsilon_{2}^{\prime}-\varepsilon_{1}\right)+4 \pi^{2} \varepsilon_{2}^{\prime \prime} V / 3 \lambda^{3}=0
$$

so that there is a small shift of the resonance to lower frequencies. More important is the broadening and the reduction in the peak value at resonance, which is now given by

$$
\left|\alpha_{\max }\right|=\frac{\varepsilon_{1} V}{4 \pi} \frac{\varepsilon_{2}^{\prime}-\varepsilon_{1}}{L \varepsilon_{2}^{\prime \prime}+4 \pi^{2}\left(\varepsilon_{1}-\varepsilon_{2}^{\prime}\right) V / 3 \lambda^{3}}
$$

This result indicates that the peak gain for large particles is independent of the particle geometry and becomes a function of frequency only, falling off rapidly at high frequencies.

The second refinement concerns the placing of the molecule near the tip of a prolate spheroid or near the edge of an oblate one. The field from the spheroid will be concentrated in these regions of high curvature and will be much larger than simply the dipolar component included in (5.41). This effect has been referred to as the "lightning rod" effect, and there is some experimental evidence that it may be important [134]. It is quite easy to calculate the enhancement of the pump field due to this effect. Again taking $E_{0}$ along the axis of the prolate spheroid, the field inside the spheroid is uniform and given by [4]

$$
E_{\mathrm{in}}=\left\{\varepsilon_{1} /\left[\varepsilon_{1}+L\left(\varepsilon_{2}-\varepsilon_{1}\right)\right]\right\} E_{0} .
$$

At the tip of the spheroid the field is normal to the surface, and therefore the field just outside can be determined from the continuity of the normal component of $\boldsymbol{D}$,

$$
E_{\text {out }}=\left\{\varepsilon_{2} /\left[\varepsilon_{1}+L\left(\varepsilon_{2}-\varepsilon_{1}\right)\right]\right\} E_{0} .
$$

Following Wokaun et al. [133], we define $f\left(\omega_{0}\right)$ as the enhancement factor for the field at $\omega_{0}$ including radiation damping. The molecular dipole induced at $\omega_{0}$ is then given by $\mu\left(\omega_{0}\right)=f\left(\omega_{0}\right) \alpha_{1} E_{0}$, where

$$
f(\omega)=\varepsilon_{2}(\omega) /\left\{\varepsilon_{1}+\left[\varepsilon_{2}(\omega)-\varepsilon_{1}\right]\left[L-i 4 \pi^{2} V / 3 \lambda^{3}\right]\right\}
$$

The dipole induced at $\omega_{\mathrm{R}}$, which is the sum of the molecular dipole $c_{\mathrm{R}} \mu\left(\omega_{0}\right)$ and the dipole induced in the spheroid, is enhanced by exactly the same factor $f$, but evaluated at $\omega_{\mathrm{R}}$ [130]. The SERS gain $G$ for a molecule at the tip of the spheroid is then

$$
G=\left|f\left(\omega_{0}\right) f\left(\omega_{\mathrm{R}}\right)\right|^{2}
$$

Calculated results of $G$ vs. $\hbar \omega_{0}$ for $\mathrm{Ag}$ spheroids of different aspect ratios and volumes are shown in fig. 31. The double-peak behavior of the solid curves (the ones for the smaller spheroids) arises from the 


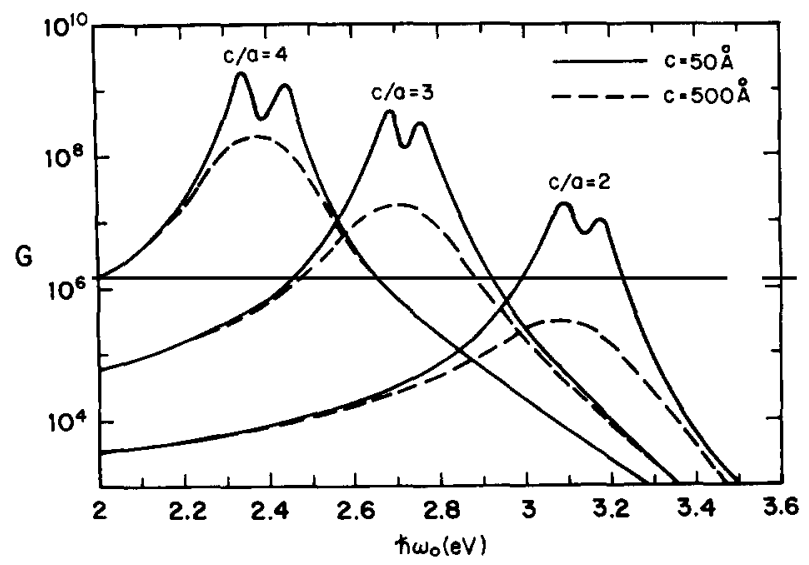

Fig. 31. Calculated Raman gain from eq. (5.56) for a molecule on the tip of an isolated prolate Ag spheroid. The Raman shift is $1000 \mathrm{~cm}^{-1}$. The solid curves are for $c=50 \mathrm{~A}$, the dashed ones for $c=500 \AA$.

fact that $G$ has a maximum when either $f\left(\omega_{0}\right)$ or $f\left(\omega_{\mathrm{R}}\right)$ has a maximum. The separation of the peaks thus represents the Raman shift. Note that, due to the radiation damping, the larger spheroids have reduced peak gains, and that all of the gain curves fall off rapidly at high frequency. To include image enhancement effects in these calculations, we simply multiply the gains calculated here by those calculated in section 5.1.

Several additional modifications to the theory must be included before results such as those in fig. 31 can be compared with experiment. The most important of these is to average (5.56) over the surface of the spheroid. This reduces the gain substantially but does not shift the resonance. As an example, for $3: 1$ oblate spheroids Liao and Stern [55] estimate the averaged gain to be about two orders of magnitude below the peak gain. Also to be included are the image field effect between the spheroid and its substrate and the interactions between the neighboring spheroids. These last two effects can be characterized by parameters $\beta_{\text {image }}$ and $\beta_{\text {int }}$ to be added to the depolarization factor L [129]. The $\beta$ parameters were originally introduced in the analysis of optical properties of metal-island films $[127,129,135,136]$. For the case of oblate spheroids (which includes most experimental situations) their effects are generally small. For prolate spheroids, however, the $\beta_{\text {int }}$ term can lead to a large positive shift of the resonance frequency as the density of particles increases.

Metal-island films show a peaked resonant optical absorbance spectrum arising from the same particle resonances discussed above. The close correlation observed experimentally between the absorbance spectra and the SERS excitation profiles in such films demonstrates convincingly that the same mechanism is operative in both cases $[52,54]$. Even more convincing evidence for the importance of the particle resonances is the demonstrated agreement between theory and experiment, both in lineshape and magnitude, for SERS excitation profiles on ordered arrays of $\mathrm{Ag}$ and $\mathrm{Au}$ particles $[55,133]$. None of these experiments, however, precludes the possibility that there is a short range (atomic scale?) mechanism that has no resonant structure and contributes a factor of 10 to 100 to the SERS gains. Indeed, there are several independent observations supporting just such a short range, possibly molecular specific enhancement. (1) Otto [94] analyzed the optical data on Ag-island films by Yamaguchi et al. $[135,136]$ and concluded that only about $10^{3}-10^{4}$ of the SERS gains in such films can be attributed to the particle resonances, while enhancements of $10^{5}-10^{6}$ are often seen. (2) Seki [53] found that Ag-island films gave SERS for pyridine but not for $\mathrm{CO}$, while cold-grown Ag samples gave SERS for both molecules. The particle resonances should enhance all molecules equally. (3) Sanda et al. 
[106] found a long-range SERS gain of about $10^{2}$ associated with the surface plasmon excitation mechanism discussed in section 5.2 and an additional very short-range effect also of about $10^{2}$ associated with the first monolayer.

It is clear from the experimental evidence that a large part of the SERS gain observed on surfaces with controlled roughness (gratings, metal islands, or particle arrays) can be attributed to electromagnetic effects of the sort discussed in this chapter. The situation for randomly roughened surfaces is less clear. However, Murray and co-workers [137,138] have observed that a long-range mechanism (50-100 $\AA$ ) exists on a variety of randomly roughened Ag surfaces. This long-range effect could arise from the particle resonances described above or from the roughness-outcoupling effect discussed in section 5.3. More likely a combination of both effects is occurring, with the former being favored on the $\mathrm{CaF}_{2}$ overgrown $\mathrm{Ag}$ layers, which are island-like, and the latter being favored on the cold-grown $\mathrm{Ag}$ films, which have a smaller roughness scale.

\section{Appendix A}

We give here a brief derivation of the identities (4.9) and (4.10) using standard references. The discussion is essentially the same as that given in ref. [42] and is repeated here for completeness. We begin by writing

$$
\exp [\mathrm{i} \boldsymbol{p} \cdot \boldsymbol{\rho}-p(z-d)]=\exp \left[-(p \hat{z}-\mathrm{i} \boldsymbol{p}) \cdot \boldsymbol{r}_{1}\right]=\sum_{l=0}^{\infty} \frac{\left(-p r_{1}\right)^{l}}{l !}\left[\cos \theta_{1}-\mathrm{i} \sin \theta_{1} \cos \left(\phi_{1}-\phi\right)\right]^{l}
$$

Now, using a formula on p. 55 of ref. [75],

$$
\begin{aligned}
{\left[\cos \theta_{1}-\mathrm{i} \sin \theta_{1} \cos \left(\phi_{1}-\phi\right)\right]^{l} } & =P_{l}\left(\cos \theta_{1}\right)+2 \sum_{m=1}^{l} \frac{\mathrm{i}^{m} l !}{(l+m) !} P_{l}^{m}\left(\cos \theta_{1}\right) \cos m\left(\phi_{1}-\phi\right) \\
& =\sum_{m=-l}^{l} \frac{i^{m} l !}{(l+m) !} P_{l}^{m}\left(\cos \theta_{1}\right) \exp \left[i m\left(\phi_{1}-\phi\right)\right]
\end{aligned}
$$

where to get this last form we have used

$$
P_{l}^{-m}(x)=(-)^{m} \frac{(l-m) !}{(l+m) !} P_{l}^{m}(x)
$$

Putting (A.2) in (A.1) we get the identity (4.9).

To get $(4.20)$ we begin with the formula

$$
P_{l}^{-m}(\cos \theta)=\frac{1}{(l+m) !} \int_{0}^{\infty} \mathrm{d} t t^{l} \mathrm{e}^{-t \cos \theta} J_{m}(t \sin \theta), \quad(0<\theta<\pi / 2)
$$

given on p. 68 of ref. [75]. Using (A.3) and the relation

$$
P_{l}^{m}(-x)=(-)^{l-m} P_{l}^{m}(x)
$$


we can write

$$
P_{l}^{m}\left(\cos \theta_{1}\right)=\frac{(-)^{l}}{(l-m) !} \int_{0}^{\infty} \mathrm{d} t t^{l} \exp \left(t \cos \theta_{1}\right) J_{m}\left(t \sin \theta_{1}\right), \quad \pi / 2<\theta_{1}<\pi
$$

We recall now the well-known integral representation of the Bessel function, given on p. 26 of ref. [75],

$$
J_{m}(z)=\frac{(-\mathrm{i})^{m}}{2 \pi} \int_{0}^{2 \pi} \mathrm{d} \phi \exp [\mathrm{i}(z \cos \phi+m \phi)]
$$

Putting this in (A.6) and making the changes of integration variables, $t \rightarrow p r_{1}, \varphi \rightarrow \phi-\phi_{1}$, we get the identity (4.10).

\section{References}

[1] L.I. Schiff, Quantum Mechanics (McGraw-Hill, New York, 1949) Ch. X.

[2] J.D. Jackson, Classical Electrodynamics, 2nd ed. (Wiley, New York 1975).

[3] L.D. Landau and E.M. Lifshitz, Electrodynamics of Continuous Media (Pergamon Press, Oxford, 1960).

[4] J.A. Stratton, Electromagnetic Theory (McGraw-Hill, New York 1941).

[5] H. Wolter, Handbuch der Physik, Vol. 24 (Springer, Berlin, 1956).

[6] F. Garcia-Moliner and F. Flores, Introduction to the theory of solid surfaces (Cambridge U. Press, Cambridge, 1979).

[7] G.E.H. Reuter and E.H. Sondheimer, Proc. Roy. Soc. (London) A195 (1948) 336.

[8] K.L. Kliewer and R. Fuchs, Phys. Rev. 172 (1978) 607.

[9] J. Lindhard, Kg. Danske Videnskab. Selskab, Mat.-Fys. Medd. 28 (1954) no. 8.

[10] C.G. Darwin, Proc. Roy. Soc. A146 (1934) 17; A182 (1943) 152.

[11] K.L. Kliewer and R. Fuchs, Phys. Rev. 181 (1969) 552.

[12] N.D. Mermin, Phys. Rev. B1 (1970) 2362.

[13] G.W. Ford (to be published).

[14] D.E. Beck and V. Celli, Nuovo Cimento B67 (1970) 230; Phys. Rev, Lett. 28 (1972) 1124.

[15] B.N.J. Persson and M. Persson, Surf. Sci. 97 (1980) 609.

[16] T. Maniv and H. Metiu, J. Chem. Phys. 72 (1980) 1906; 76 (1982) 696, 2697;

G. Korzeniewski, T. Maniv and H. Metiu, Chem. Phys. Lett. 73 (1980) 212; J. Chem. Phys. 76 (1982) 1564.

[17] C. Kittel, Quantum theory of solids (Wiley, New York, 1963).

[18] D. Pines, Elementary excitations in solids (Benjamin, New York, 1964).

[19] P.J. Feibelman, Prog. Surf. Sci. 12 (1982) 287.

[20] P.J. Feibelman, Phys. Rev. Lett. 43 (1979) 952.

[21] P.J. Feibelman, Phys. Rev. B22 (1980) 3654.

[22] P.J. Feibeiman, Phys. Rev. B14 (1976) 762; Phys. Rev. B12 (1975) 1319.

[23] R.R. Chance, A. Prock and R. Silbey, Adv. Chem. Phys. 37 (1978) 1-65.

[24] K.H. Drexhage, J. Lumin. 1/2 (1970) 693.

[25] K.H. Drexhage, in: Progress in Optics XII, ed. E. Wolf,. (North-Holland, Amsterdam, 1974) p. 165.

[26] H. Morawitz, Phys. Rev. 187 (1969) 1792.

[27] H. Kuhn, J. Chem. Phys. 53 (1970) 101.

[28] K.H. Tews, Ann. Phys. Leipz. 29 (1973) 97.

[29] R.R. Chance, A. Prock and R. Silbey, J. Chem. Phys. 60 (1974) 2744.

[30] H. Morawitz and M.R. Philpott, Phys. Rev. B10 (1974) 4863.

[31] M.R. Philpott, J. Chem. Phys. 62 (1975) 1812.

[32] Y.M. Gerbshtein, I.A. Merkulov, and D.N. Mirlin, JETP Lett. 22 (1975) 35.

[33] W.H. Weber and C.F. Eagen and Opt. Lett. 4 (1979) 239.

[34] R.E. Benner, R. Dornhaus and R.K. Chang, Opt. Commun. 30 (1979) 149. 
[35] I. Pockrand, A. Brillante and D. Mobius, Chem. Phys. Lett. 69 (1980) 499.

[36] A. Adams, J. Moreland, P.K. Hansma and Z. Schlesinger, Phys. Rev. B25 (1982) 3457.

[37] C.F. Eagen, W.H. Weber, S.L. McCarthy and R.W. Terhune, Chem Phys. Lett. 75 (1980) 274.

[38] A. Campion, A.R. Gallo, C.B. Harris, H.J. Robota and P.M. Whitmore, Chem. Phys. Lett. 73 (1980) 447.

[39] A. Sommerfeld, Ann. Phys. 28 (1909) 665.

[40] A. Sommerfeld, Partial differential equations (Academic Press, New York, 1979) Ch. VI

[41] H. Weyl, Ann. Phys. 60 (1919) 481.

[42] G.W. Ford and W.H. Weber, Surf. Sci. 109 (1981) 451.

[43] W.H. Weber and G.W. Ford, Phys. Rev. Lett. 44 (1980) 1774

[44] J.E. Sipe, Surf. Sci. 105 (1981) 489.

[45] W. Lukosz, Phys. Rev. B22 (1980) 3030; J. Opt. Soc. Am. 71 (1981) 744.

[46] M. Otter, Z. Phys. 161 (1961) 163; M.M. Dujardin and M.L. Theye, J. Phys. Chem. Solids 32 (1971) 2033; W.H. Weber and S.L. McCarthy, Phys. Rev. B12 (1975) 5643.

[47] J.C. Tsang, J.R. Kirtley and J.A. Bradley, Phys. Rev. Lett 43 (1979) 772; J.C. Tsang, J.R. Kirtley, T.N. Theis and S.S. Jha, Phys. Rev. B25 (1982) 5070.

[48] J. Lambe and S.L. McCarthy, Phys. Rev. Lett 37 (1976) 923; S.L. McCarthy and J. Lambe, Appl. Phys. Lett 30 (1977) 427.

[49] L.C. Davis, Phys. Rev. B16 (1977) 2842.

[50] B. Laks and D.L. Mills, Phys. Rev. B21 (1980) 5175.

[51] J.G. Bergman. D.S. Chemla, P.F. Liao, A.M. Glass, R.H. Hart and D.H. Olson, Opt. Lett. 6 (1981) 33; A.M. Glass, P.F. Liao, J.G. Bergman and D.H. Olson, Opt. Lett 5 (1980) 368.

[52] D.A. Weitz, S. Garoff, C.D. Hanson, T.J. Gramila and J.I. Gersten, Opt. Lett. 7 (1982) 89; D.A. Weitz, S. Garoff and T.J. Gramila, Opt. Lett. 7 (1982) 168.

[53] H. Seki and M.R. Philpott, J. Chem. Phys. 73 (1980) 5376; H. Seki, J. Chem. Phys. 76 (1982) 4412.

[54] R.M. Hart, J.G. Bergman and A. Wokaun, Opt. Lett. 7 (1982) 105.

[55] P.F. Liao and M.B. Stern, Opt. Lett. 7 (1982) 483.

[56] J.A. Creighton, C.G. Blatchford and M.G. Albrecht, Trans. Faraday Soc. II, 75 (1979) 790.

[57] M. Kerker, O. Siiman, L.A. Bumm and D.S. Wang, Appl. Opt. 19 (1980) 3253.

[58] O. Siiman, L.A. Bumm, R. Callagham, C.G. Blatchford and M. Kerker, J. Phys. Chem. 87 (1983) 1014

[59] K.V. Von Raben, R.K. Chang and B.L. Laube, Chem. Phys. Lett 79 (1981) 465.

[60] S.L. McCall, P.M. Platzmann and P.A. Wolff, Phys. Lett. 77A (1980) 381.

[61] M. Kerker, D.-S. Wang and H. Chew, Appl. Opt. 19 (1980) 4159.

[62] P.W. Barber, R.K. Chang and H. Massoudi, Phys. Rev. B27 (1983) 7251.

[63] E.W. Hobson, The Theory of Spherical and Ellipsoidal Harmonics (Cambridge Univ. Press, Cambridge 1931) p. 139.

[64] H. Faxen, Ann. Phys. 68 (1922) 89.

[65] F. Delanaye, A.A. Lucas and G.D. Mahan, in: Proc. 7th Vacuum Congr. and 3rd Intern. Conf. on Solid Surfaces, Vienna, 1977, p. 477.

[66] G.D. Mahan and A.A. Lucas, J. Chem. Phys. 68 (1978) 1344.

[67] M. Scheffler, Surface Sci. 81 (1979) 562.

[68] N. Sheppard and T.T. Nguyen, in: Advances in Infrared and Raman Spectroscopy, eds. R.J.H. Clark and R.E. Hester (Heyden, London, 1978) Ch. 2, p. 67.

[69] F.B. Hildebrand, Methods of Applied Mathematics, 2nd ed. (Prentice-Hall, Englewood Cliffs, NJ, 1962).

[70] E.T. Whittaker and G.N. Watson, Modern Analysis, 4th ed. (Cambridge University Press, Cambridge, 1952).

[71] L.D. Landau and E.M. Lifshitz, Statistical Physics, 2nd ed. (Addison-Wesley, Reading MA, 1969).

[72] E.U. Condon and G.H. Shortley, Theory of Atomic Spectra (Cambridge University Press, Cambridge, 1957).

[73] C.A. Coulson, Proc. Roy. Soc. Edinburgh 61A (1941) 20.

[74] R.M. Sternheimer, Phys. Rev. A1 (1970) 321.

[75] W. Magnus and F. Oberhettinger, Formulas and Theorems for the Special Functions of Mathematical Physics (Chelsea, New York, 1949).

[76] G.D. Mahan, Solid State Ionics 1 (1980) 29.

[77] G.W. Ford and W.H. Weber, Surf. Sci. 129 (1983) 123.

[78] S. Efrima and H. Metiu, Surf. Sci. 92 (1980) 433; 108 (1981) 329.

[79] P. Hollins and J. Pritchard, Chem. Phys. Lett. 75 (1980) 378.

[80] I.L. Fabelinskii, Molecular Scattering of Light (Plenum, New York, 1968) pp. 555-560.

[81] P.B. Johnson and R.W. Christy, Phys. Rev. B12 (1972) 4370.

[82] C.F. Eagen and W.H. Weber, Phys. Rev. B19 (1979) 5068.

[83] H. Ehrenreich and H.R. Philipp, Phys. Rev. 128 (1962) 1622.

[84] N.W. Ashcroft and N.D. Mermin, Solid State Physics (Holt, Reinhart, and Winston, New York, 1976) p. 391.

[85] P. Apell, Physica Scripta 24 (1981) 795. 
[86] N.D. Lang and W. Kohn, Phys. Rev. B1 (1970) 4555.

[87] B.N.J. Persson and N.D. Lang, Phys. Rev. B26 (1982) 5409.

[88] H. Goldstein, Classical Mechanics, 2nd ed. (Addison-Wesley, Reading, Mass., 1980) Ch. 6.

[89] S. Anderson and B.N.J. Persson, Phys. Rev. Lett. 45 (1980) 1421.

[90] H. Huber, E.P. Kundig, M. Moskovits and G.A. Ozin, J. Am. Chem. Soc. 97 (1975) 2097.

[91] B.N.J. Persson and M. Persson, Solid State Commun. 36 (1980) 175.

[92] R.W. Stobie, B. Rao and M.J. Dignam, Surf. Sci. 56 (1976) 334.

[93] P. Hollins and J, Pritchard, Surf. Sci. 89 (1979) 486.

[94] A. Otto, in: Light Scattering in Solids, Vol. IV, eds. M. Cardona and G. Güntherodt (Springer, Berlin 1983) pp. $289-418$.

[95] H. Metiu, Progress in Surf. Science (to be published).

[96] R.P. Van Duyne, in: Chemical and Biological Applications of Lasers, Vol. 4, ed. C.B. Moore (Academic Press, New York 1979) Ch. 5.

[97] Surface Enhanced Raman Scattering, eds. R.K. Chang and T.E. Furtak (Plenum Press, New York, 1982).

[98] F.W. King, R.P. Van Duyne and G.C. Schatz, J. Chem. Phys. 69 (1978) 4472.

[99] S. Efrima and H. Metiu, J. Chem. Phys. 70 (1979) 1602 and 2297; Surf. Sci. 92 (1980) 417.

[100] R. Naanan, S.J. Buelow, O. Chesnovsky and D.R. Herschbach, J. Phys. Chem. 84 (1980) 2692.

[101] E.G. Wilson and S.A. Rice, in: Optical Properties and Electronic Structure of Metals and Alloys, ed. F. Abèles (Wiley, New York, 1966$) 271$.

[102] G.W. Ford and W.H. Weber, Surf. Sci. 110 (1981) L587.

[103] G.L. Eesley and J.R. Smith, Solid State Commun. 31 (1979) 815

[104] P.R. Hilton and D.W. Oxtoby, J. Chem. Phys. 72 (1980) 6346.

[105] A. Girlando, M.R. Philpott, D. Heitmann, J.D. Swalen and R. Santo, J. Chem. Phys. 72 (1980) 5187.

[106] P.N. Sanda, J.M. Warlaumont, J.E. Demuth, J.C. Tsang, K. Christmann and J.A. Bradley, Phys. Rev. Lett. 45 (1980) 1519.

[107] J.C. Tsang, J.R. Kirtley and T.N. Theis, Solid State Commun. 35 (1980) 667.

[108] M. Yamashita and M. Tsuji, J. Phys. Soc. Jap. 52 (1983) 2462.

[109] M. Nevière and R. Reinisch, Phys. Rev. B26 (1982) 5403.

[110] D.L. Mills and M. Weber, Phys. Rev. B26 (1982) 1075.

[111] S.S. Jha, J.R. Kirtley and J.C. Tsang, Phys. Rev. B22 (1980) 3973.

[112] A. Otto, Z. Phys. 216 (1968) 398.

[113] B. Pettinger, A. Tadjeddine and D.M. Kolb, Chem. Phys. Lett. 66 (1979) 544.

[114] For recent reviews see the articles by H. Raether (Ch. 9) and A.A. Maradudin (Ch. 10), in: Surface Polaritons, eds. V.M. Agranovich and D.L. Mills (North-Holland, Amsterdam, 1982)

[115] W.H. Weber and G.W. Ford, Opt. Lett. 6 (1981) 122.

[116] R.H. Ritchie, E.T. Arakawa, J.J. Cowan and R.N. Hamm, Phys. Rev. Lett. 21 (1968) 1530.

[117] C.K. Chen, Ph.D. thesis, University of California, Berkeley, 1980 (unpublished).

[118] M.C. Hutley and D. Maystre, Opt. Commun. 19 (1976) 431.

[119] R.M. Hexter and M.G. Albrecht, Spectrochim, Acta 35A (1979) 233.

[120] J.C. Tsang, J.R. Kirtley and J.A. Bradley, Phys. Rev. Lett. 43 (1979) 772.

[121] E. Burstein, J. Chen, C.Y. Chen, S. Lundquist and E. Tosatti, Solid State Commun. 29 (1979) 567.

[122] J.L. Stanford, J. Opt. Soc. Am. 60 (1970) 49.

[123] S.O. Sari, D.K. Cohen and K.D. Scherkoske, Phys. Rev. B21 (1980) 2162.

[124] B. Pettinger, V. Wenning and D.M. Kolb, Ber. Bunsenges. Phys. Chem. 82 (1978) 1236.

[125] E. Kröger and E. Kretschmann, Z. Phys. 237 (1970) 1.

[126] E. Kretschmann, Z. Phys. 227 (1969) 412.

[127] C.F. Eagen, Appl. Opt. 20 (1981) 3035.

[128] C.Y. Chen, I. Davoli, G. Ritchie and E. Burstein, Surf. Sci. 101 (1980) 363.

[129] C.Y. Chen and E. Burstein, Phys. Rev. Lett. 45 (1980) 1287.

[130] J.I. Gersten and A. Nitzan, J. Chem. Phys. 73 (1980) 3023.

[131] D.-S. Wang and M. Kerker, Phys. Rev. B24 (1981) 1777.

[132] P.C. Das and J.I. Gersten, Phys. Rev. B25 (1982) 6281.

[133] A. Wokaun, J.P. Gordon and P.F. Liao, Phys. Rev. Lett. 48 (1982) 957.

[134] D.A. Weitz, T.J. Gramila, A.Z. Genack and J.I. Gersten, Phys. Rev. Lett. 45 (1980) 355.

[135] T. Yamaguchi, S. Yoshida and A. Kinbara, J. Opt. Soc. Am. 64 (1974) 1563.

[136] T. Yamaguchi, S. Yoshida and A. Kinbara, Thin Solid Films 21 (1974) 173.

[137] C.A. Murray, D.L. Allara, A.F. Hegard and F.J. Padden, Jr., Surf. Sci. 119 (1982) 449.

[138] C.A. Murray, D.L. Allara and M. Rhinewine, Phys. Rev, Lett. 46 (1981) 57;

J.E. Rowe, C.V. Shank, D.A. Zwemer and C.A. Murray, Phys. Rev. Lett. 44 (1980) 1770. 